\title{
The Genus Solanum: An Ethnopharmacological, Phytochemical and Biological Properties Review
}

\author{
Joseph Sakah Kaunda ${ }^{1,2} \cdot$ Ying-Jun Zhang ${ }^{1,3}$ (D)
}

Received: 3 January 2019 / Accepted: 27 February 2019 / Published online: 12 March 2019

(c) The Author(s) 2019

\begin{abstract}
Over the past 30 years, the genus Solanum has received considerable attention in chemical and biological studies. Solanum is the largest genus in the family Solanaceae, comprising of about 2000 species distributed in the subtropical and tropical regions of Africa, Australia, and parts of Asia, e.g., China, India and Japan. Many of them are economically significant species. Previous phytochemical investigations on Solanum species led to the identification of steroidal saponins, steroidal alkaloids, terpenes, flavonoids, lignans, sterols, phenolic comopunds, coumarins, amongst other compounds. Many species belonging to this genus present huge range of pharmacological activities such as cytotoxicity to different tumors as breast cancer (4T1 and EMT), colorectal cancer (HCT116, HT29, and SW480), and prostate cancer (DU145) cell lines. The biological activities have been attributed to a number of steroidal saponins, steroidal alkaloids and phenols. This review features 65 phytochemically studied species of Solanum between 1990 and 2018, fetched from SciFinder, Pubmed, ScienceDirect, Wikipedia and Baidu, using "Solanum" and the species' names as search terms ("all fields").
\end{abstract}

Keywords Solanum $\cdot$ Solanaceae $\cdot$ Phytochemistry $\cdot$ Steroidal saponins and alkaloids $\cdot$ Ethnopharmacology

\begin{tabular}{|c|c|}
\hline \multicolumn{2}{|c|}{ Abbreviations } \\
\hline ABTS & $\begin{array}{l}\text { 2,2'-Azino-bis(3-ethylbenzthiazoline-6-sulphonic } \\
\text { acid) }\end{array}$ \\
\hline $\mathrm{CC}_{50}$ & $\begin{array}{l}\text { Cytotoxic concentration of the extracts to cause } \\
\text { death to } 50 \% \text { of host's viable cells }\end{array}$ \\
\hline CDDP & cis-Diamminedichloroplatinum \\
\hline DPPH & 2,2-Diphenyl-1-picrylhydrazyl \\
\hline EC50 & Half maximal effective concentration \\
\hline GABA & Neurotransmitter gamma-aminobutyric acid \\
\hline HBV & Hepatitis B Virus \\
\hline HSV-1 & Herpes simplex virus type 1 \\
\hline IC50 & $\begin{array}{l}\text { Minimum inhibition concentration for inhibiting } \\
50 \% \text { of the pathogen }\end{array}$ \\
\hline
\end{tabular}

Ying-Jun Zhang

zhangyj@mail.kib.ac.cn

1 State Key Laboratory of Phytochemistry and Plant Resources in West China, Kunming Institute of Botany, Chinese Academy of Sciences, Kunming 650204, People's Republic of China

2 Graduate School of the Chinese Academy of Sciences, Beijing 100039, People's Republic of China

3 Yunnan Key Laboratory of Natural Medicinal Chemistry, Kunming Institute of Botany, Chinese Academy of Sciences, Kunming 650201, People's Republic of China
LD50 Dose required to kill half the members of a tested population after test duration

MIC Minimum inhibitory concentration

MTT 3-(4,5-dimethylthiazol-2-yl)-2,5-diphenyltetrazolium bromide

SAG Superoxide anion generation

\section{Introduction}

The genus Solanum is considered to be one of the largest and most complex genera among the Angiosperms [1], and the most representative and largest genus of the family Solanaceae [1-4]. It is comprised of about 2000 species distributed across subtropical and tropical regions of Asia [3-9], tropical Africa [10-29], non-arid Africa [30-43], Americas [44-87], Australia [71-74, 81-84] and India [71]. The genus is well represented in Brazil with about 350 species widely distributed from north to south in diverse phytogeographic regions [70, 80]. In Brazil (Ceará, Bahia, Mato Grosso do Sul, Paraná and north-central coast of Santa Catarina State), many Solanum species, usually known as 'yubeba', the word that refers to the prickles found on the stems of several of the species, are widely used in traditional medicine $[66,80,87]$. In the northeast 
of Brazil, 80 Solanum species are distributed throughout the region and used in folk medicine. One of such species is $S$. capsicoides, commonly known as "Gogoia" [87]. In East Africa, several Solanum species such as $S$. arundo and $S$. incanum are known to be poisonous and are reportedly used to induce miscarriages [64].

Solanum genus is rich in economically significant species; the food crops include $S$. aethiopicum [20, 21], S. anguivi $[30,31]$ S. lycopersicum, S. melongena, S. muricatum, $S$. torvum and $S$. tuberosum [1]. Ornamental species include S. aviculare, S. capsicastrum, S. crispum, S. laciniatum, $S$. laxum, S. pseudocapsicum, S. rantonnetii, S. seaforthianum and $S$. wendlandii [1].

A series of pharmacological studies have been carried out to verify and validate the traditional medicinal applications of many plants in this genus. The studied pharmacological activities include analgesic, anthelminthic, antiallergic, anti-anemic, anti-asthmatic, antibacterial, anti- cancer, anti-convulsant, anti-depressant, anti-diabetic, anti-fungal, antihistaminic, antihyperten- sive, anti-inflammatory, antileishmanial, antimelanogenetic, anti-molluscicidal, antinociceptive, anti-psoriatic, antiplasmodial, antiprotozoa, anti-trypanosomal, antiurolithiatic, antiviral, cardio- vascular, diuretic, hepatoprotective, hypolipidemic, mosquito larvicidal, nephrotoxic, spasmolytic, schistosomicidal and vasorelaxant activities.

In the past, several reviews on Solanum genus have been documented [88-101], however, mostly with singular focus on particular species. The present review is multi faceted, and features 66 medicinal species of Solanum in their geographical distribution, traditional uses, and 670 isolated chemical constituents, including 134 steroidal saponins, 63 steroidal alkaloids, 13 pregnane glycosides, 128 terpenes, 75 flavonoids, 31 lignans, 31 other types of alkaloids, 66 sterols, 52 phenolic compounds, 20 coumarins and coumestans, 4 coumarinolignoids, 23 fatty acids and esters and 30 other compounds. Where applicable, the biological activities of compounds isolated from various species are noted.

\section{Distribution and Ethnopharmacological Uses}

Sixty-six species commonly used as important folk medicine, ornamental plants, or wild food sources were selected in this review, and their local names, distribution and ethnopharmacologi- cal uses were summarized in Table 1. Local names are given in different languages with which the inhabitants of a particular region use to identify a specific species. Each species' natural habitat and/or places of cultivation are mentioned. Traditional as well as modern day applications are presented.

\section{Chemical Constituents and Their Biological Properties}

At least 670 compounds, including 134 steroidal saponins (1-134), 63 steroidal alkaloids (135-197), 13 pregnane glycosides (198-210), 128 terpenes (211-338), 72 flavonoids (339-413), 31 lignans (414-444), 31 other types of alkaloids (445-475), 66 sterols (476-541), 52 phenols (542-593), 20 coumarins and coumestans (594-613), 4 coumarinolignoids (614-617), 23 fatty acids and esters (618-640) and 30 other compounds (641-670) were reported from the genus Solanum. Most of them were investigated for various biological activities. The chemical constituents and their biological properties are presented in Table 2, together with their plant sources and parts, alongside the classification of structures.

\subsection{Steroidal Saponins}

Steroidal saponins are prominent characteristic components in Solanum species, from which 134 compounds, 1-134, have been obtained (Fig. 1). Among all the studied species, $S$. torvum was the one studied mostly, resulting in the isolation of 32 saponins including chlorogenone (1), (5 $2,25 S)$-spirostan-3,6-dione (2), diosgenone (13), 56-72, neochlorogenin (73), solanolactosides A-C (91-93), torvosides J-L (95-97) and 98-102 from the leaves, fruits, aerial parts and the whole plant [323, 325, 430, 435, 436, $448,449,451,452,463]$.

Included herein are spirostane saponins, SC1-SC6 (35-40), isolated from the leaves of $S$. chrysotrichum [113-115, 117], and lyconosides Ia (46), Ib (47), II (48), III (49), and IV (50) reported from the fruits of S. lycocarpum. Indiosides $\mathrm{G}(\mathbf{8 2})$ and $\mathrm{H}(\mathbf{8 3})$ with an iso-type $\mathrm{F}$ ring were isolated from the methanolic extract of the whole plant of S. violaceum, together with indioside I (86), and two unusual furostanol saponins with a deformed F ring, indiosides J (87) and K (88) [391, 392]. In addition, four steroidal sapogenins, indiosides $\mathrm{L}-\mathrm{O}(\mathbf{7 8}-\mathbf{8 1})$ were also obtained from this plant [391]. Indioside L (78) is a rare spirostanoid possessing a 1,4-dien-3-one moiety in ring A. Compounds 80 and $\mathbf{8 1}$ represent rare examples of spirostane with the $3 \beta, 7 \alpha$-diol-5,6-ene moiety compared to the normal 3 $\beta, 7 \beta$-diol-5,6-ene derivatives [391].

Two C-22 steroidal lactone saponins, namely solanolactosides A, B $(\mathbf{9 1}, \mathbf{9 2})$ and two spirostanol glycosides, torvosides $\mathrm{M}, \mathrm{N}(\mathbf{2 3}, \mathbf{8})$ were isolated from ethanol extract of aerial parts of S. torvum. Compounds $\mathbf{9 1}$ and $\mathbf{9 2}$ possess the aglycon of solanolide (94), while $\mathbf{2 3}$ and $\mathbf{8}$ have the aglycons of yamogenin (76) and neochlorogenin (73), resp. The aglycon of 94 is an unusual C-22 steroidal lactone sapogenin [316]. 


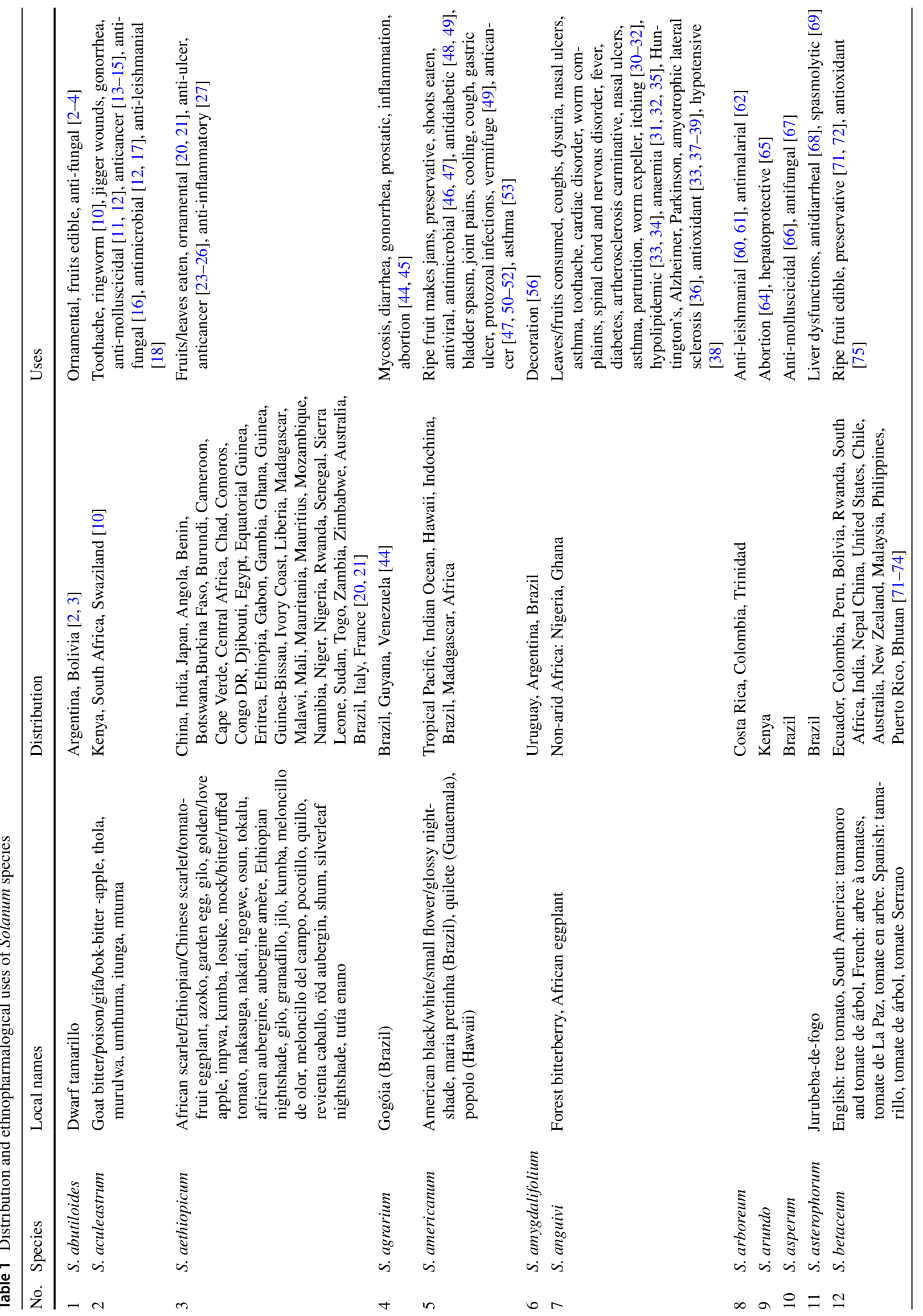




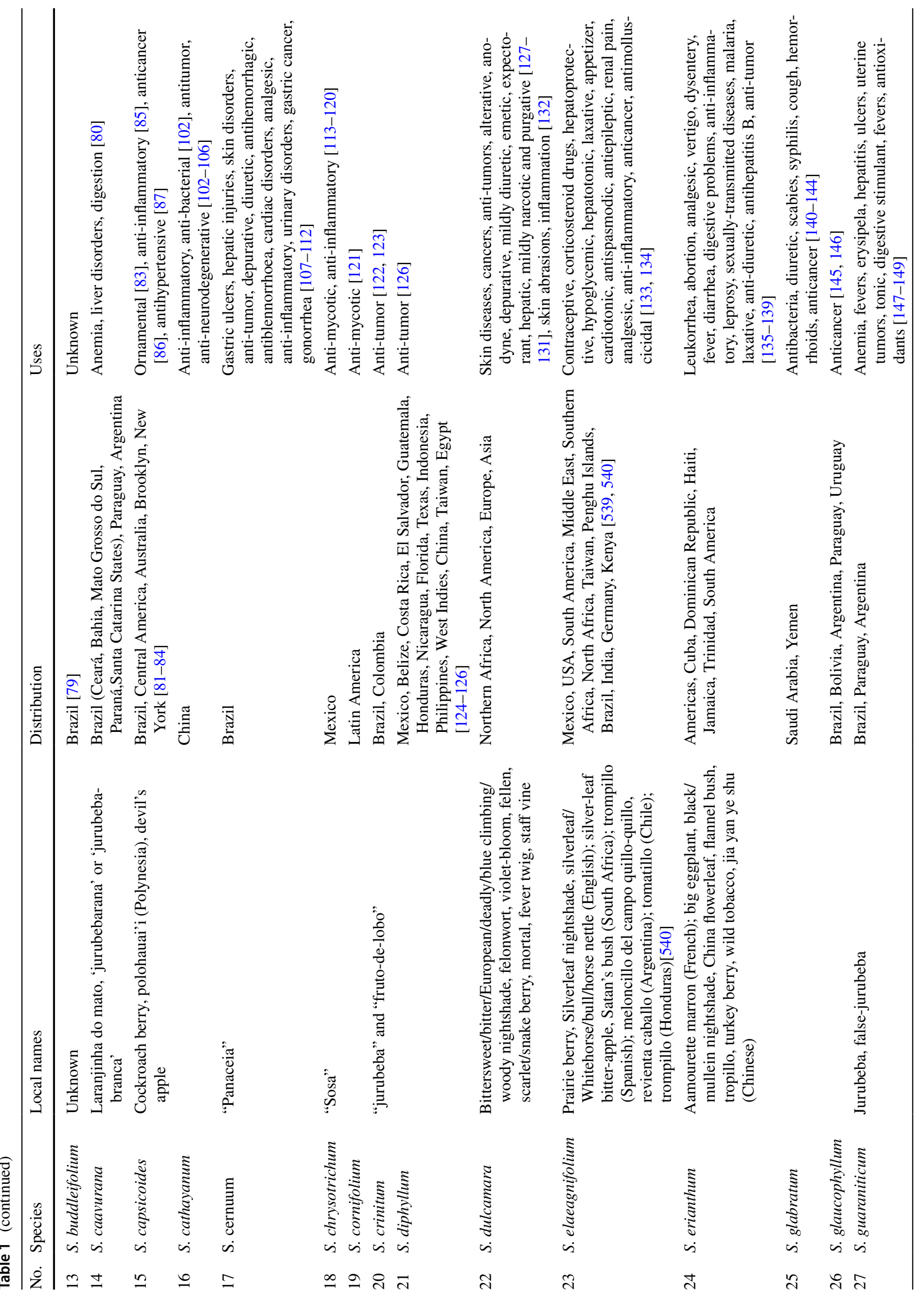




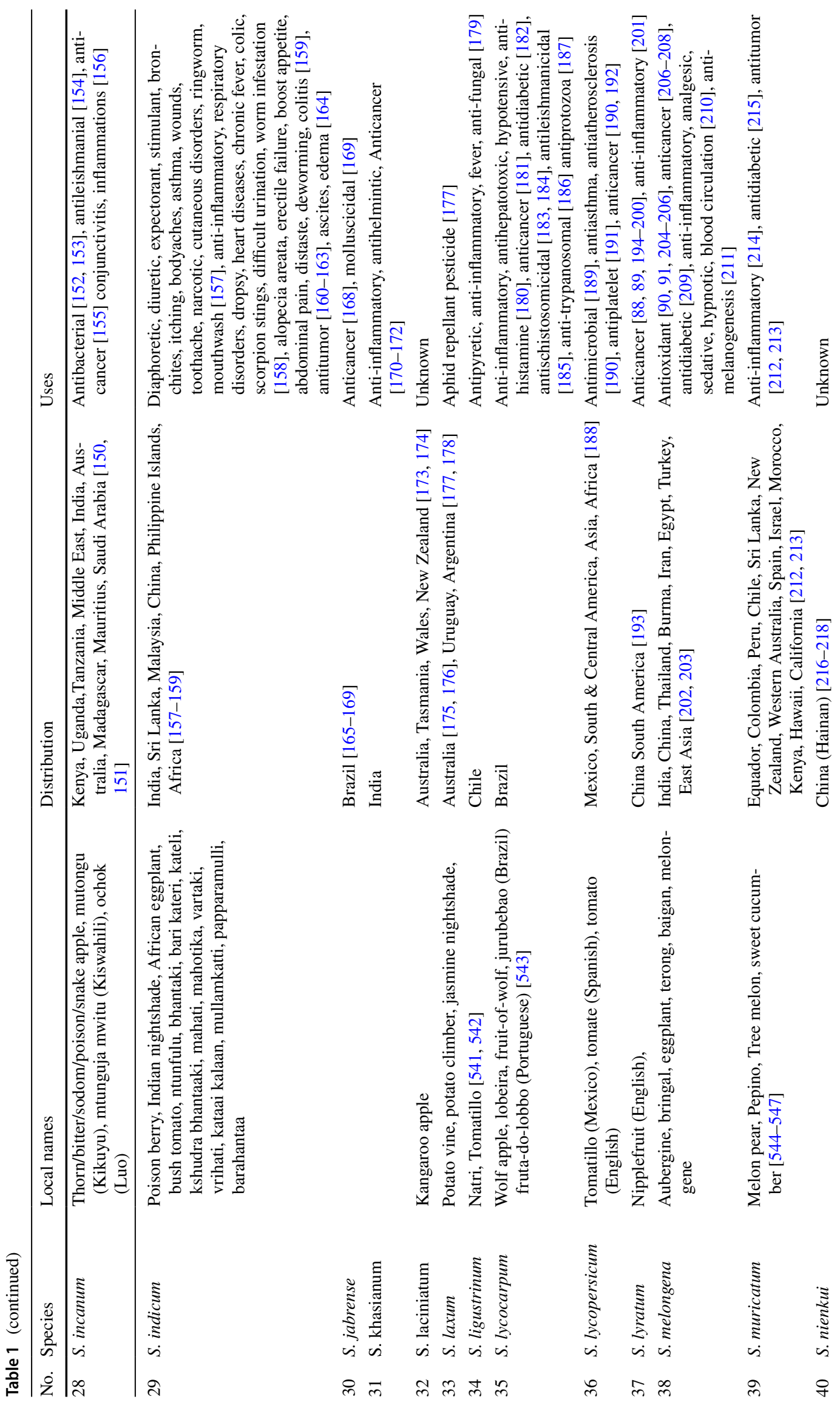




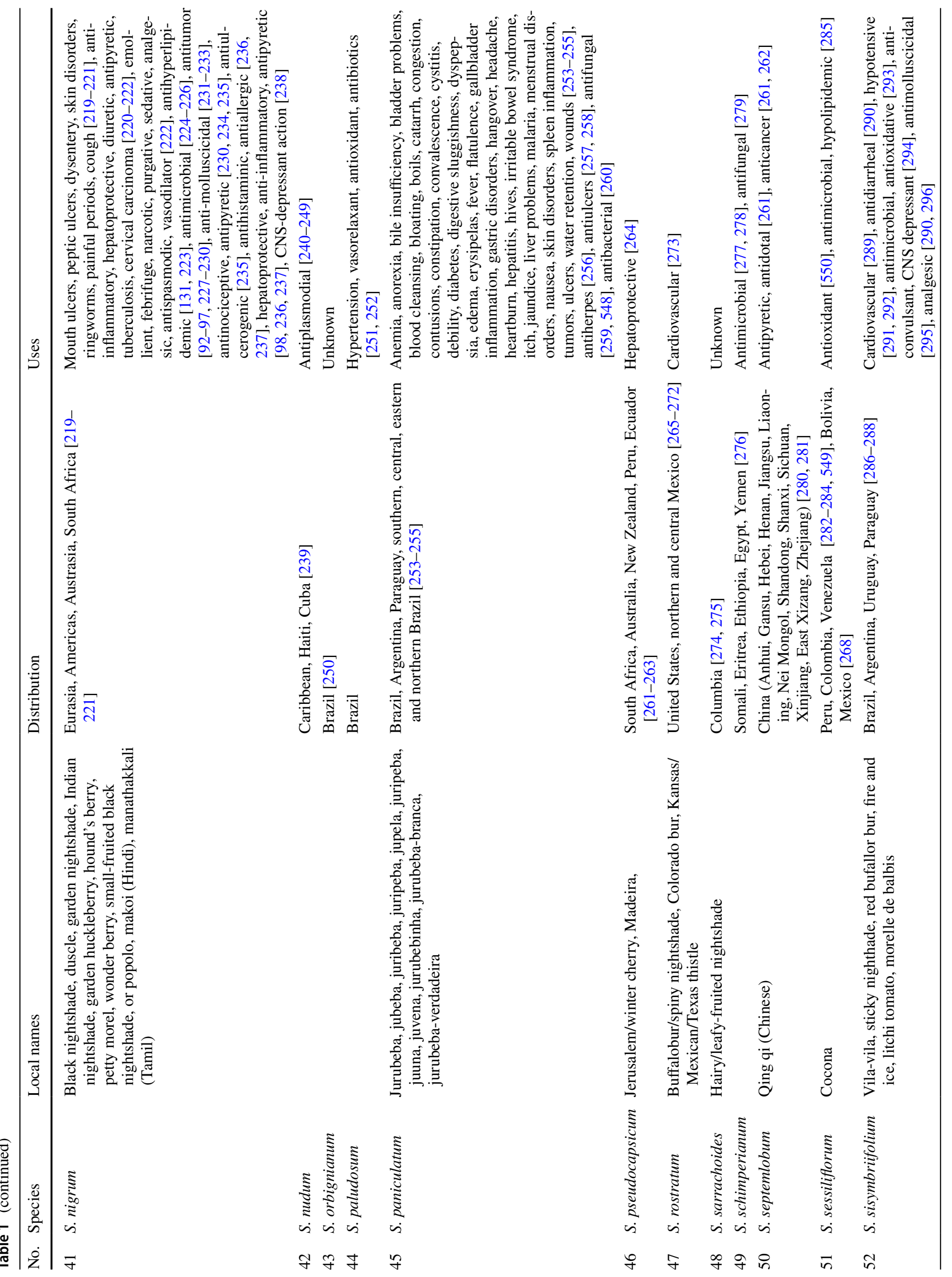




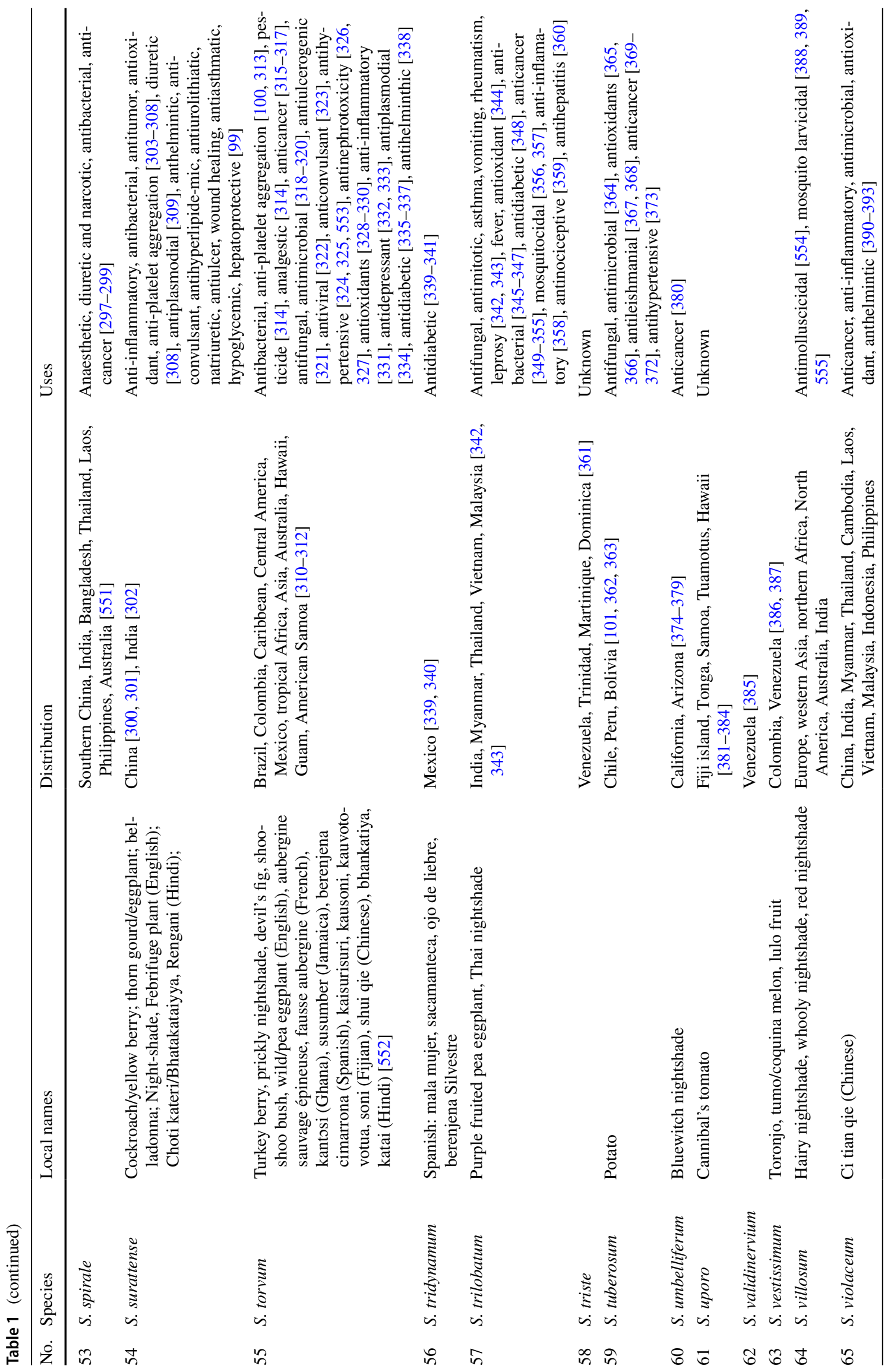




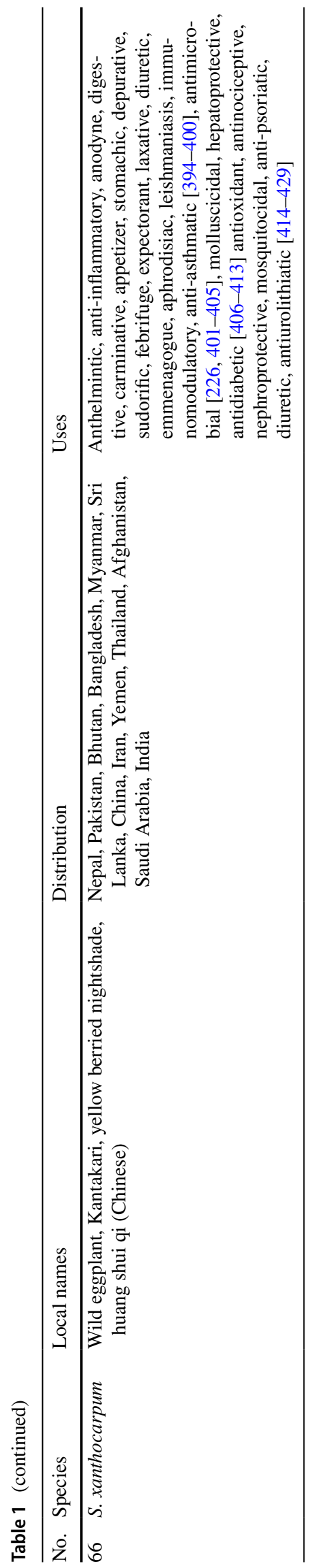

椈 Springer 
Table 2 Phytochemistry, biological properties and classification of Solanum compounds

\begin{tabular}{|c|c|c|c|c|c|}
\hline No. & Compounds & Plant sources & Parts & Biological properties & References \\
\hline & \multicolumn{5}{|l|}{ Steroidal Saponins } \\
\hline 1 & Chlorogenone & S. torvum & Fruit & & {$[430]$} \\
\hline 2 & $(5 \alpha, 25 S)$-Spirostan-3,6-dione & S. torvum & Fruit & & [430] \\
\hline 3 & Solakhasoside & S. khasianum & Fruit & & [431] \\
\hline 4 & Foliumin & S. amygdalifolium & Aerial & & {$[57]$} \\
\hline 5 & Foliumin A & S. amygdalifolium & Aerial & & {$[56]$} \\
\hline 6 & Neotigogenin & S. paniculatum & Leaf & Cytotoxic & [257] \\
\hline 7 & Diuranthoside A & S. cathayanum & Root & & [432] \\
\hline 8 & Torvoside N & S. torvum & Aerial & Anticancer & [316] \\
\hline 9 & Atroposide E & S. dulcamara & Aerial & & [433] \\
\hline 10 & Degalactotigonin & S. dulcamara & Aerial & & [433] \\
\hline 11 & Trillin & S. paniculatum & Aerial & & [258] \\
\hline 12 & Diosgenin gentiobioside & S. paniculatum & Aerial & & [258] \\
\hline 13 & Diosgenone & S. nudum & Leaf & Hepatoprotective & {$[242,247,249]$} \\
\hline 14 & $\begin{array}{l}(22 R, 23 S, 25 R)-3 \beta, 6 \alpha, 23 \text {-trihydroxy- } \\
5 \alpha \text {-spirostane 6- } O-\beta \text {-D-xylosyl- }\left(1^{\prime \prime \prime}-\right. \\
\left.\text { 3"')- } O \text {-[ } \beta \text {-D-quinovosyl( }\left(1^{\prime \prime \prime}-2 '\right)\right]-O \text {-[ } \alpha \text {-L- } \\
\text { rhamnosyl }\left(1^{\prime \prime}-3 \text { ')] }-O-\beta \text {-D-quinovoside }\right.\end{array}$ & S. paniculatum & Aerial & & {$[258]$} \\
\hline 15 & Nuatigenosido & S. sisymbriifolium & Root & Antihypertensive & {$[289,291]$} \\
\hline 16 & $\begin{array}{l}(3 \beta, 5 \alpha, 14 \beta, 25 R)-3 \text {-Hydroxyspirost- } 8 \text {-en- } \\
\text { 11-one }\end{array}$ & S. villosum & Leaf & & [434] \\
\hline 17 & $\begin{array}{l}(3 \beta, 5 \alpha, 6 \alpha, 25 S) \text {-3-Hydroxyspirostan-6-yl } \\
\text { 6-deoxy-3- } O \text {-(6-deoxy- } \alpha \text {-L-mannosyl) } \\
\text { - } \beta \text {-D-glucoside }\end{array}$ & S. torvum & Whole & & [435] \\
\hline 18 & Torvoside Q & S. torvum & Aerial & & {$[331,436]$} \\
\hline \multirow[t]{3}{*}{19} & \multirow[t]{3}{*}{ Dioscin } & S. indicum & Fruit & & {$[160]$} \\
\hline & & S. melongena & Fruit & Antimelanogenesis & [211] \\
\hline & & S. rostratum & Aerial & & [437] \\
\hline 20 & Prosapogenin A & S. indicum & Fruit & & {$[160]$} \\
\hline \multirow[t]{7}{*}{21} & \multirow[t]{7}{*}{ Diosgenin } & S. lycopersicum & Aerial & & [438] \\
\hline & & S. melongena & Aerial & & [439] \\
\hline & & S. nigrum & Fruit & & [440] \\
\hline & & S. torvum & Fruit & & [430] \\
\hline & & S. tridynamum & Root & & [341] \\
\hline & & S. tuberosum & Stem & & [441] \\
\hline & & S. violaceum & Aerial & & {$[391,442]$} \\
\hline 22 & Aspidistrin & S. cathayanum & Root & & {$[432]$} \\
\hline 23 & Torvoside M & S. torvum & Aerial & Anticancer & [316] \\
\hline \multirow[t]{4}{*}{24} & \multirow[t]{4}{*}{ Protodioscin } & S. abutiloides & Root & & [7] \\
\hline & & S. incanum & Root & & [156] \\
\hline & & S. indicum & Fruit & & {$[160,443]$} \\
\hline & & S. spirale & Fruit & & {$[444]$} \\
\hline \multirow[t]{2}{*}{25} & \multirow[t]{2}{*}{ Methylprotodioscin } & S. incanum & Root & & {$[155]$} \\
\hline & & S. indicum & Fruit & & [160] \\
\hline 26 & Indioside D & S. incanum & Root & & [156] \\
\hline \multirow[t]{2}{*}{27} & \multirow{2}{*}{$\begin{array}{l}\text { 26- } O \text { - } \beta \text {-D-Glucosyl-22-methoxyfurost-5- } \\
\text { ene-3 } \beta, 26 \text {-diol 3- } O \text { - } \alpha \text {-L-rhamnosyl-(1- } \\
\text { 2)- } \beta \text {-D-glucoside }\end{array}$} & S. indicum & Fruit & & {$[160]$} \\
\hline & & S. spirale & Fruit & & [444] \\
\hline 28 & $\begin{array}{l}(3 \beta, 22 \alpha, 25 R)-26-(\beta \text {-D-Glucosyloxy)- } \\
\text { 22-hydroxyfurost-5-en-3-yl } O-\beta \text {-D- } \\
\text { glucosyl-(1-2)- } O \text { - } \beta \text {-D-glucosyl-(1-4)- } \beta \text { - } \\
\text { D-glucoside }\end{array}$ & S. cathayanum & Root & & [432] \\
\hline
\end{tabular}


Table 2 (continued)

\begin{tabular}{|c|c|c|c|c|c|}
\hline No. & Compounds & Plant sources & Parts & Biological properties & References \\
\hline 29 & 25R-Timosaponin $\mathrm{H} 1$ & S. cathayanum & Root & & [432] \\
\hline 30 & Torvoside $\mathrm{O}$ & S. torvum & Leaf & & {$[445]$} \\
\hline 31 & $\begin{array}{l}(23 S, 25 R) \text {-spirost-5-en-3,23 diol 3- } O \text { - } \alpha \text { - } \\
\text { L-rhamnosyl-(1-2)- } O \text { - } \alpha \text {-L-rhamnosyl-1- } \\
\text { 4) } \beta \text {-D-glucoside }\end{array}$ & S. glabratum & Aerial & & {$[141]$} \\
\hline 32 & $\begin{array}{l}\text { 23- } \beta \text {-D-glucosyl }(23 S, 25 R) \text { spirost- } \\
\text { 5-en-3,23 diol 3- } O \text { - } \alpha \text {-L-rhamnosyl-1- } \\
\text { 2) } O \text { - } \alpha \text {-L-rhamnosyl-(1-4) } \beta \text {-D-glucoside }\end{array}$ & S. glabratum & Aerial & & [141] \\
\hline 33 & $\begin{array}{l}(25 R) \text { spirost-5-en-3-ol 3- } O-\alpha \text {-L- } \\
\text { rhamnosyl-1-2) } O-\beta \text {-D-glucosyl-1-3) } \beta \text {-D- } \\
\text { galactoside }\end{array}$ & S. glabratum & Aerial & & [141] \\
\hline 34 & Isonuatigenin-3- $O-\beta$-solatriose & S. sisymbriifolium & Root & & [446] \\
\hline 35 & Saponin SC-1 & S. chrysotrichum & Leaf & & [118] \\
\hline 36 & Saponin SC-2 & S. chrysotrichum & Leaf & Antifungal & {$[113-115,117]$} \\
\hline 37 & Saponin SC-3 & S. chrysotrichum & Leaf & Antifungal & {$[114,117]$} \\
\hline 38 & Saponin SC-4 & S. chrysotrichum & Leaf & Antifungal & {$[114,117]$} \\
\hline 39 & Saponin SC-5 & S. chrysotrichum & Leaf & Antifungal & {$[114,117]$} \\
\hline 40 & Saponin SC-6 & $\begin{array}{l}\text { S. chrysotrichum } \\
\text { S. torvum }\end{array}$ & $\begin{array}{l}\text { Leaf } \\
\text { Whole }\end{array}$ & Antifungal & $\begin{array}{l}{[114,117]} \\
{[435]}\end{array}$ \\
\hline 41 & Chlorogenin & $\begin{array}{l}\text { S. chrysotrichum } \\
\text { S. tridynamum } \\
\text { S. torvum }\end{array}$ & $\begin{array}{l}\text { Leaf } \\
\text { Root } \\
\text { Fruit }\end{array}$ & & $\begin{array}{l}{[117]} \\
{[341]} \\
{[430]}\end{array}$ \\
\hline 42 & Chrysogenin & S. chrysotrichum & Leaf & & [117] \\
\hline 43 & Laxumin A & S. laxum & Aerial & & [178] \\
\hline 44 & Laxumin B & S. laxum & Aerial & & [178] \\
\hline 45 & Luciamin & S. laxum & Aerial & & {$[177]$} \\
\hline 46 & Lyconoside Ia & S. lycocarpum & Fruit & & {$[447]$} \\
\hline 47 & Lyconoside Ib & S. lycocarpum & Fruit & & {$[447]$} \\
\hline 48 & Lyconoside II & S. lycocarpum & Fruit & & {$[447]$} \\
\hline 49 & Lyconoside III & S. lycocarpum & Fruit & & [447] \\
\hline 50 & Lyconoside IV & S. lycocarpum & Fruit & & [447] \\
\hline 51 & $\begin{array}{l}\text { 26- } O \text {-( } \beta \text {-D-Glucosyl) nuatigenin-3- } O-\alpha \text {-L- } \\
\text { rhamnosyl-(1-4)- } \beta \text {-D-glucoside }\end{array}$ & S. surattense & Aerial & & {$[305]$} \\
\hline 52 & Aculeatiside A & S. surattense & Aerial & & [305] \\
\hline 53 & $\begin{array}{l}(22 \mathrm{R}, 23 \mathrm{~S}, 25 \mathrm{R})-3 \beta, 6 \alpha, 23 \text {-trihydroxy- } \\
5 \alpha \text {-spirostane } 6-O-\beta \text {-D-xylosyl-(1-3) } \\
\text { - } \beta \text {-D-quinovoside }\end{array}$ & S. surattense & Aerial & & {$[305]$} \\
\hline 54 & $\begin{array}{l}(22 \mathrm{R}, 23 \mathrm{~S}, 25 \mathrm{~S})-3 \beta, 6 \alpha, 23 \text {-trihydroxy- } 5 \alpha- \\
\text { spirostane } 6-O-\beta-\mathrm{D}-\mathrm{xyl} \text { - } \\
\text { quinovoside }(1-3)-O-\beta-\mathrm{D}-\end{array}$ & S. surattense & Aerial & & {$[305]$} \\
\hline 55 & $\begin{array}{l}(22 \mathrm{R}, 23 \mathrm{R}, 25 \mathrm{~S})-3 \beta, 6 \alpha, 23 \text {-trihydroxy- } 5 \alpha- \\
\text { spirostane } 6-O-\beta-\mathrm{D}-\mathrm{xyl} \text { - } \\
\text { quinovoside }(1-3)-O-\beta \text {-D- }\end{array}$ & S. surattense & Aerial & & {$[305]$} \\
\hline 56 & Neochlorogenin 6- $O-\beta$-D-quinovoside & S. torvum & Aerial & & {$[331,448]$} \\
\hline 57 & $\begin{array}{l}\text { Neochlorogenin 6- } O-\beta \text {-D-xylosyl } \\
\text {-(1-3)- } \beta \text {-D-quinovoside }\end{array}$ & S. torvum & Aerial & Anti-inflammatory & {$[331,448]$} \\
\hline 58 & $\begin{array}{l}\text { Neochlorogenin 6- } O \text { - } \alpha \text {-L-rhamnosyl-(1-3)- } \\
\beta \text {-D-quinovoside }\end{array}$ & S. torvum & Aerial & & {$[448,449]$} \\
\hline 59 & Solagenin $6-O-\beta$-D-quinovoside & S. torvum & Whole & & {$[448-450]$} \\
\hline 60 & $\begin{array}{l}\text { Solagenin 6- } O-\alpha \text {-L-rhamnosyl-(1-3)- } \beta \text {-D- } \\
\text { quinovoside }\end{array}$ & S. torvum & Whole & & [448] \\
\hline 61 & $\begin{array}{l}(25 S) 26-\beta \text {-D-glucosyloxy) } 3 \text {-oxo- } 5 \alpha \text { - } \\
\text { furost-20(22)en- } 6 \alpha \text {-yl- } O \text { - } \beta \text {-D-xyloside }\end{array}$ & S. torvum & Fruit & & [451] \\
\hline
\end{tabular}


Table 2 (continued)

\begin{tabular}{|c|c|c|c|c|c|}
\hline No. & Compounds & Plant sources & Parts & Biological properties & References \\
\hline 62 & $\begin{array}{l}(25 S) 26-\beta \text {-D-glucosyloxy) } 3 \text {-oxo- } 22 \alpha- \\
\text { methoxy-5 } \alpha \text {-furostan- } 6 \alpha \text {-yl- } O-\beta \text {-D- } \\
\text { xyloside }\end{array}$ & S. torvum & Fruit & & [451] \\
\hline 63 & $\begin{array}{l}(25 S) 26-\beta \text {-D-glucosyloxy) } 3 \beta \text {-hydroxy- } \\
22 \alpha \text {-methoxy- } 5 \alpha \text {-furostan- } 6 \alpha \text {-yl- } O \text { - } \alpha \text {-L- } \\
\text { rhamnosyl-1-3) } \beta \text {-D-glucoside }\end{array}$ & S. torvum & Fruit & & {$[451]$} \\
\hline 64 & Torvoside A & S. torvum & Aerial & & {$[313,449]$} \\
\hline 65 & Torvoside B & S. torvum & Root & & [449] \\
\hline 66 & Torvoside E & S. torvum & Root & & [449] \\
\hline 67 & Torvoside F & S. torvum & Root & & [449] \\
\hline 68 & Torvoside H & S. torvum & Fruit & & [313] \\
\hline 69 & $\begin{array}{l}\text { (25S)3 } 3 \beta \text {-hydroxy- } 5 \alpha \text {-spirostan- } 6 \alpha \text {-yl- } O-\beta \text { - } \\
\text { D-xyloside }\end{array}$ & S. torvum & Fruit & & {$[451]$} \\
\hline 70 & $\begin{array}{l}(25 S) 3 \text {-oxo- } 5 \alpha \text {-spirostan- } 6 \alpha \text {-yl- } O-\beta \text {-D- } \\
\text { xyloside }\end{array}$ & S. torvum & Fruit & & {$[451]$} \\
\hline 71 & 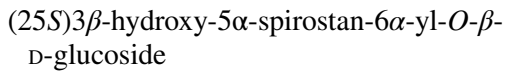 & S. torvum & Fruit & & [451] \\
\hline 72 & $\begin{array}{l}\text { (25S)3 } 3,27 \text {-dihydroxy- } 5 \alpha \text {-spirostan- } 6 \alpha \text {-yl- } \\
O-\beta \text {-D-glucoside. }\end{array}$ & S. torvum & Fruit & & [451] \\
\hline \multirow[t]{2}{*}{73} & \multirow[t]{2}{*}{ Neochlorogenin } & S. tridynamum & Root & & {$[451]$} \\
\hline & & S. torvum & Aerial & & [341] \\
\hline \multirow[t]{2}{*}{74} & \multirow[t]{2}{*}{ Tigogenin } & S. americanum & Leaf & & [54] \\
\hline & & S. torvum & Fruit & & {$[430]$} \\
\hline 75 & Yuccagenin & S. tridynamum & Root & & [341] \\
\hline 76 & Yamogenin & S. violaceum & Aerial & & [391] \\
\hline 77 & Yamogenone & S. violaceum & Aerial & & [391] \\
\hline 78 & Indioside L & S. violaceum & Aerial & & [391] \\
\hline 79 & Indioside $\mathrm{M}$ & S. violaceum & Aerial & & [391] \\
\hline 80 & Indioside $\mathrm{N}$ & S. violaceum & Aerial & & [391] \\
\hline 81 & Indioside $\mathrm{O}$ & S. violaceum & Aerial & & [391] \\
\hline 82 & Indioside $\mathrm{G}$ & S. violaceum & Whole & & [392] \\
\hline 83 & Indioside $\mathrm{H}$ & S. violaceum & Whole & Anticancer & [392] \\
\hline 84 & Borassoside D & S. violaceum & Whole & & [392] \\
\hline 85 & Borassoside E & S. violaceum & Whole & $\begin{array}{l}\text { Anticancer, anti-inflam- } \\
\text { matory }\end{array}$ & [392] \\
\hline 86 & Indioside I & S. violaceum & Whole & $\begin{array}{l}\text { Anticancer, anti-inflam- } \\
\text { matory }\end{array}$ & [392] \\
\hline 87 & Indioside J & S. violaceum & Whole & & [392] \\
\hline 88 & Indioside $\mathrm{K}$ & S. violaceum & Whole & & [392] \\
\hline \multirow[t]{2}{*}{89} & \multirow[t]{2}{*}{ Yamoscin } & S. torvum & Aerial & Anti-inflammatory & [331] \\
\hline & & S. violaceum & Whole & Anticancer & [392] \\
\hline 90 & Zingiberoside A1 & S. violaceum & Whole & & [392] \\
\hline 91 & Solanolactoside A & S. torvum & Aerial & & [316] \\
\hline 92 & Solanolactoside B & S. torvum & Aerial & & [316] \\
\hline 93 & Solanolactoside C & S. torvum & Aerial & & [436] \\
\hline 94 & Solanolide & S. torvum & Aerial & & [316] \\
\hline \multirow[t]{2}{*}{95} & \multirow[t]{2}{*}{ Torvoside J } & S. surattense & Aerial & Anticonvulsant & [305] \\
\hline & & S. torvum & Aerial & & {$[323,331,452]$} \\
\hline \multirow[t]{2}{*}{96} & \multirow[t]{2}{*}{ Torvoside K } & S. surattense & Aerial & Anticonvulsant, antifungal & [305] \\
\hline & & S. torvum & Aerial & & {$[323,331,452]$} \\
\hline
\end{tabular}


Table 2 (continued)

\begin{tabular}{|c|c|c|c|c|c|}
\hline No. & Compounds & Plant sources & Parts & Biological properties & References \\
\hline \multirow[t]{3}{*}{97} & \multirow[t]{3}{*}{ Torvoside L } & S. surattense & Aerial & \multirow[t]{4}{*}{ Anticonvulsant } & [305] \\
\hline & & S. torvum & Aerial & & $\begin{array}{l}{[323,331,435,} \\
452]\end{array}$ \\
\hline & & S. paniculatum & Leaf & & [260] \\
\hline 98 & $\begin{array}{l}(22 \mathrm{R}, 23 \mathrm{~S}, 25 \mathrm{~S})-3 \beta, 6 \alpha, 23 \text {-trihydroxy- } 5 \alpha- \\
\text { spirostane } 6-O-\beta-\mathrm{D}-\mathrm{xylosyl}-(1-3)-O-\beta \text {-D- } \\
\text { quinovoside }\end{array}$ & S. torvum & Aerial & & {$[323,331]$} \\
\hline 99 & $\begin{array}{l}(22 \mathrm{R}, 23 \mathrm{~S}, 25 \mathrm{R})-3 \beta, 6 \alpha, 23 \text {-trihydroxy-5 } \alpha- \\
\text { spirostane } 6-O-\beta-\mathrm{D}-\mathrm{xylosyl}-(1-3)-O-\beta \text {-D- } \\
\text { quinovoside }\end{array}$ & S. torvum & Aerial & Anti-inflammatory & [331] \\
\hline 100 & $\begin{array}{l}\text { (22R,23R,25S)-3 } \beta, 6 \alpha, 23 \text {-trihydroxy- } 5 \alpha- \\
\text { spirostane } 6-O-\beta \text {-D-xylosyl-(1-3)- } O \text { - } \beta \text {-D- } \\
\text { quinovoside }\end{array}$ & S. torvum & Aerial & Anti-inflammatory & {$[331]$} \\
\hline 101 & Gekogenin & S. torvum & Fruit & & [430] \\
\hline 102 & Sisalagenin & S. torvum & Fruit & & [430] \\
\hline 103 & $\Delta^{25(27)}$ tigogenin-3- $O-\beta$-D-glucoside & S. paniculatum & Leaf & Antiviral & [257] \\
\hline 104 & Soladulcosides A & S. dulcamara & Aerial & & [129] \\
\hline 105 & Soladulcosides B & S. dulcamara & Aerial & & [129] \\
\hline 106 & Abutiloside L & S. abutiloides & Root & & [4] \\
\hline 107 & Abutiloside M & S. abutiloides & Root & & [4] \\
\hline 108 & Abutiloside N & S. abutiloides & Root & & [4] \\
\hline 109 & Abutiloside O & S. abutiloides & Root & & {$[4]$} \\
\hline 110 & Torvoside C & S. torvum & Root & & [449] \\
\hline \multirow[t]{2}{*}{111} & \multirow[t]{2}{*}{ Torvoside D } & S. surattense & Aerial & & [305] \\
\hline & & S. torvum & Root & & {$[331,449]$} \\
\hline 112 & Torvoside G & S. torvum & Fruit, Root & & {$[313,449]$} \\
\hline 113 & Torvoside P & S. torvum & Leaf & & [445] \\
\hline 114 & Anguivioside A & S. anguivi & Fruit & & {$[41]$} \\
\hline 115 & Anguivioside B & S. anguivi & Fruit & & [41] \\
\hline 116 & Anguivioside $\mathrm{C}$ & S. anguivi & Fruit & & [41] \\
\hline 117 & Anguivioside I & S. indicum & Fruit & & [443] \\
\hline \multirow[t]{2}{*}{118} & \multirow[t]{2}{*}{ Anguivioside III } & S. anguivi & Fruit & & [43] \\
\hline & & S. indicum & Fruit & & [443] \\
\hline 119 & Anguivioside XI & S. anguivi & Fruit & & [43] \\
\hline 120 & Anguivioside XV & S. anguivi & Fruit & & [43] \\
\hline 121 & Anguivioside XVI & S. anguivi & Fruit & & [43] \\
\hline 122 & Inunigroside A & S. nigrum & Fruit & & [453] \\
\hline 123 & $\begin{array}{l}25(S)-26-O-\beta \text {-D-glucosyl- } 5 \alpha \text {-furost- } \\
22(20) \text {-en- } 3 \beta, 6 \alpha, 26 \text {-triol 6- } O \text { - }[\alpha \text {-L- } \\
\text { rhamnosyl-(1-3)- } O \text { - } \beta \text {-D-quinovoside }]\end{array}$ & S. torvum & Fruit & Anticancer & [317] \\
\hline 124 & $\begin{array}{l}\text { 25(S)-26- } O \text { - } \beta \text {-D-glucosyl-5 } \alpha \text {-furost- } \\
22(20) \text {-en-3-one- } 6 \alpha, 26 \text {-diol 6- } O \text { - }[\alpha \text {-L- } \\
\text { rhamnosyl-(1-3)- } O \text { - } \beta \text {-D-quinovoside }]\end{array}$ & S. torvum & Fruit & Anticancer & [317] \\
\hline 125 & $\begin{array}{l}25(S) \text { - } 26-O-\beta \text {-D-glucosyl- } 5 \alpha \text {-furost- } \\
22(20) \text {-en- } 3 \beta, 6 \alpha, 26 \text {-triol 6- } O \text { - } \beta \text {-D- } \\
\text { quinovoside }\end{array}$ & S. torvum & Fruit & Anticancer & [317] \\
\hline 126 & Paniculonin B & S. torvum & Leaf & & [323] \\
\hline 127 & Smilaxchinoside A & S. rostratum & Aerial & & [437] \\
\hline 128 & 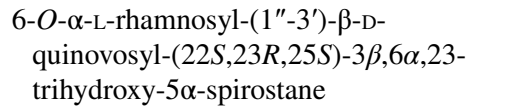 & S. paniculatum & Leaf & & [260] \\
\hline
\end{tabular}


Table 2 (continued)

\begin{tabular}{|c|c|c|c|c|c|}
\hline No. & Compounds & Plant sources & Parts & Biological properties & References \\
\hline 129 & $\begin{array}{l}\text { 6- } O-\beta \text {-D-Xylosyl- }\left(1^{\prime \prime}-3^{\prime}\right)-\beta \text {-D-quinovosyl- } \\
(23 R, 25 S)-3 \beta, 6 \alpha, 23 \text {-trihydroxy- } 5 \alpha- \\
\text { spirostane }\end{array}$ & S. paniculatum & Leaf & & {$[260]$} \\
\hline 130 & $\begin{array}{l}\text { 6- } O-\beta \text {-D-Xylosyl-(1"-3')- } \beta \text {-D-quinovosyl- } \\
\text { ( } 22 S, 23 R, 25 R)-3 \beta, 6 \alpha, 23 \text {-trihydroxy-5 } \alpha \text { - } \\
\text { spirostane }\end{array}$ & S. paniculatum & Leaf & & {$[260]$} \\
\hline 131 & 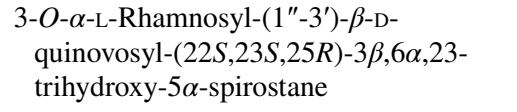 & S. paniculatum & Leaf & & [260] \\
\hline 132 & $\begin{array}{l}\text { 3- } O-\beta \text {-D-Xylosyl- }\left(1^{\prime \prime}-3^{\prime}\right)-\beta \text {-D-quinovosyl- } \\
\quad(22 S, 23 S, 25 R)-3 \beta, 6 \alpha, 23 \text {-trihydroxy-5 } \alpha \text { - } \\
\text { spirostane }\end{array}$ & S. paniculatum & Leaf & & {$[260]$} \\
\hline 133 & 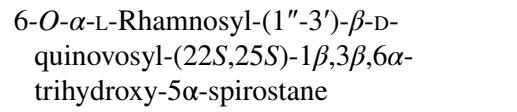 & S. paniculatum & Leaf & & {$[260]$} \\
\hline \multirow[t]{2}{*}{134} & $\begin{array}{l}\text { 6- } O-\beta \text {-D-Xylosyl- }\left(1^{\prime \prime}-3^{\prime}\right)-\beta \text {-D-quinovosyl- } \\
(22 \mathrm{~S}, 25 S)-3 \beta, 4 \beta, 6 \alpha \text {-trihydroxy- } 5 \alpha \text { - } \\
\text { spirostane }\end{array}$ & S. paniculatum & Leaf & & {$[260]$} \\
\hline & Steroidal alkaloids & & & & \\
\hline 135 & Demissine & S. tuberosum & Stem & & [101] \\
\hline 136 & Solasodiene & S. torvum & Fruit & & [430] \\
\hline 137 & Solanoside A & S. surattense & Whole & & [454] \\
\hline 138 & Solanoside B & S. surattense & Whole & & [454] \\
\hline \multirow[t]{18}{*}{139} & Solamargine & S. abutiloides & Root & & [7] \\
\hline & & S. aculeastrum & Fruit & & [19] \\
\hline & & S. asperum & Root & & {$[66,67]$} \\
\hline & & S. buddleifolium & Stem & & [79] \\
\hline & & S. americanum & Fruit & & {$[55]$} \\
\hline & & S. anguivi & Root & & {$[42]$} \\
\hline & & S. crinitum & Fruit & & [122] \\
\hline & & S. erianthum & Leaf & & {$[137,455]$} \\
\hline & & S. incanum & Root & & [156] \\
\hline & & S. khasianum & Fruit & & {$[456]$} \\
\hline & & S. lycocarpum & Fruit & $\begin{array}{l}\text { Leishmanicidal, antidia- } \\
\text { betic, schistosomicidal, } \\
\text { trypanocidal }\end{array}$ & $\begin{array}{c}{[182,183,185} \\
186,447,457]\end{array}$ \\
\hline & & S. melongena & Fruit,Root & & {$[206,439]$} \\
\hline & & S. nigrum & Whole & & [228] \\
\hline & & S. paludosum & Fruit & & {$[253]$} \\
\hline & & S. sarrachoides & Leaf & Anticancer & {$[458]$} \\
\hline & & S. surattense & Aerial & & [305] \\
\hline & & S. uporo & Root & Antibacterial, molluscicidal & [384] \\
\hline & & S. xanthocarpum & Fruit & & {$[403,406]$} \\
\hline \multirow[t]{2}{*}{140} & $\gamma$-Solamargine & S. nigrum & Whole & & [228] \\
\hline & & S. umbelliferum & Whole & & {$[380]$} \\
\hline \multirow[t]{4}{*}{141} & Khasianine & S. khasianum & Fruit & & {$[456]$} \\
\hline & & S. nigrum & Whole & & [228] \\
\hline & & S. surattense & Aerial & Anticancer & [305] \\
\hline & & S. xanthocarpum & Fruit & Antibacterial, molluscicidal & {$[403,406,407]$} \\
\hline
\end{tabular}


Table 2 (continued)

\begin{tabular}{|c|c|c|c|c|c|}
\hline No. & Compounds & Plant sources & Parts & Biological properties & References \\
\hline \multirow[t]{11}{*}{142} & \multirow[t]{11}{*}{ Solasonine } & S. americanum & Leaf & & [54] \\
\hline & & S. amygdalifolium & Aerial & & {$[56]$} \\
\hline & & S. asperum & Fruit & & {$[66,67]$} \\
\hline & & S. crinitum & Aerial & & {$[122,123,459]$} \\
\hline & & S. erianthum & Leaf & & {$[137,455]$} \\
\hline & & S. khasianum & Fruit & & {$[456]$} \\
\hline & & S. lycocarpum & Fruit & $\begin{array}{l}\text { Leishmanicidal,antidiabetic, } \\
\text { schistosomicidal }\end{array}$ & $\begin{array}{l}{[182,183,185} \\
447,457]\end{array}$ \\
\hline & & S. melongena & Fruit,Root & & {$[206,439]$} \\
\hline & & S. sarrachoides & Leaf & & [458] \\
\hline & & S. sessiliflorum & Fruit & & [460] \\
\hline & & S. sisymbriifolium & Fruit & & [294] \\
\hline 143 & $\beta 1$-Solasonine & S. nigrum & Whole & & [228] \\
\hline 144 & 12-Hydroxysolasonine & S. lycocarpum & Fruit & & {$[182,447]$} \\
\hline \multirow[t]{15}{*}{145} & \multirow[t]{15}{*}{ Solasodine } & S. americanum & Leaf & & [54] \\
\hline & & S. aculeastrum & Fruit & Anticancer & [13] \\
\hline & & S. crinitum & Aerial & & [123] \\
\hline & & S. khasianum & Fruit & & {$[172,456]$} \\
\hline & & S. laciniatum & Aerial & & {$[461,462]$} \\
\hline & & S. lycocarpum & Fruit & & [185] \\
\hline & & S. melongena & Fruit & & [206] \\
\hline & & S. nigrum & Whole & & {$[163,440]$} \\
\hline & & S. sisymbriifolium & Fruit & & [294] \\
\hline & & S. surattense & Whole & CNS depressant & [303] \\
\hline & & S. torvum & Whole & Anti-inflammatory & [463] \\
\hline & & S. trilobatum & Whole & & [358] \\
\hline & & S. villosum & Whole & & [442] \\
\hline & & S. xanthocarpum & Fruit & Antibacterial & {$[403,429]$} \\
\hline & & S. umbelliferum & Whole & & [380] \\
\hline 146 & $N$-Hydroxysolasodine & S. paludosum & Root & & [464] \\
\hline 147 & $O$-Acetylsolasodine & S. umbelliferum & Whole & & [380] \\
\hline 148 & Putuline & S. paludosum & Root & & [464] \\
\hline \multirow[t]{2}{*}{149} & \multirow[t]{2}{*}{ Anguivine } & S. anguivi & Root & & {$[42]$} \\
\hline & & S. uporo & Root & & [384] \\
\hline 150 & Isoanguivine & S. uporo & Root & & [384] \\
\hline 151 & Arudonine & S. arundo & Root & & [64] \\
\hline 152 & Solanandaine & S. asperum & Fruit & & {$[66]$} \\
\hline 153 & Robeneoside A & S. lycocarpum & Fruit & & {$[182,447]$} \\
\hline 154 & Robeneoside B & S. lycocarpum & Fruit & & {$[182,447]$} \\
\hline 155 & Lobofrutoside & S. lycocarpum & Fruit & & [447] \\
\hline 156 & Solanigroside $\mathrm{P}$ & S. nigrum & Whole & & [228] \\
\hline 157 & 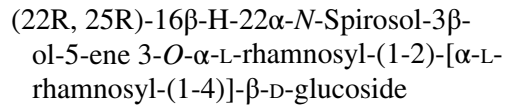 & S. surattense & Aerial & Anticancer & [305] \\
\hline 158 & Solaculine A & S. aculeastrum & Root & & [19] \\
\hline \multirow[t]{3}{*}{159} & \multirow[t]{3}{*}{$\beta$-Solamarine } & S. aculeastrum & Root & & [19] \\
\hline & & S. elaeagnifolium & Seed & & [465] \\
\hline & & S. incanum & Root & & [155] \\
\hline
\end{tabular}


Table 2 (continued)

\begin{tabular}{|c|c|c|c|c|c|}
\hline No. & Compounds & Plant sources & Parts & Biological properties & References \\
\hline \multirow[t]{4}{*}{160} & \multirow[t]{4}{*}{ Tomatidenol } & S. aculeastrum & Root & & [19] \\
\hline & & S. palodusum & Root & & [464] \\
\hline & & S. lycopersicum & Fruit & & [192] \\
\hline & & S. surattense & Aerial & & [454] \\
\hline 161 & Tomatidine 3- $O-\beta$-D-glucoside & S. arboreum & Aerial & & [63] \\
\hline 162 & Dehydrotomatine & S. lycopersicum & Fruit & & [192] \\
\hline 163 & $\begin{array}{l}\text { Tomatidine } 3-O-O-\beta \text {-D-xylosyl-1-6) } \beta \text {-D- } \\
\text { glucoside] }\end{array}$ & S. arboreum & Aerial & & [63] \\
\hline 164 & Solaverol A & S. uporo & Root & & [384] \\
\hline 165 & (23S)-23-hydroxyanguivine & S. uporo & Root & & [384] \\
\hline 166 & (23S)-23-hydroxyisoanguivine & S. uporo & Root & & [384] \\
\hline \multirow[t]{2}{*}{167} & \multirow[t]{2}{*}{ Tomatidine } & S. lycopersicum & Fruit & & [192] \\
\hline & & S. aculeastrum & Fruit & Anticancer & [13] \\
\hline \multirow[t]{3}{*}{168} & \multirow[t]{3}{*}{ Tomatine } & S. lycopersicum & Fruit & & {$[192,466]$} \\
\hline & & S. cathayanum & Whole & Neurotoxicity & [106] \\
\hline & & S. sarrachoides & Leaf & & [276] \\
\hline 169 & $\begin{array}{l}\text { 22-Imido-3-[4'-(6"-deoxy- } \alpha \text {-L- } \\
\text { mannoside)- } \beta \text {-D-glucoside]-5-dehydro } \\
\text { spirostane }\end{array}$ & S. xanthocarpum & Fruit & & [407] \\
\hline \multirow[t]{2}{*}{170} & \multirow[t]{2}{*}{ Leptinidine } & S. paludosum & Root & & [253] \\
\hline & & S. orbignianum & Aerial & & [250] \\
\hline 171 & Leptinine I & S. orbignianum & Aerial & & {$[250]$} \\
\hline 172 & Leptinine II & S. orbignianum & Aerial & & [250] \\
\hline \multirow[t]{4}{*}{173} & \multirow[t]{4}{*}{ Solanine } & S. dulcamara & Stem & & [467] \\
\hline & & S. indicum & Whole & & {$[162]$} \\
\hline & & S. tuberosum & Stem & & [441] \\
\hline & & S. villosum & Fruit & & [468] \\
\hline 174 & $\alpha$-Chaconine & S. tuberosum & Stem & & {$[372,441]$} \\
\hline 175 & $\begin{array}{l}\beta \text {-D-Glucoside, }(3 \beta, 23 \beta) 23 \text { - } \\
\text { hydroxysolanid-5-en-3-yl }\end{array}$ & S. orbignianum & Aerial & & [250] \\
\hline 176 & Solanidine & S. villosum & Fruit & & [469] \\
\hline 177 & Solanopubamine & S. schimperianum & Aerial & Antifungal & [279] \\
\hline 178 & Jurubine & S. paniculatum & Fruit & & {$[273,548]$} \\
\hline 179 & Etioline & S. spirale & Root & & [470] \\
\hline \multirow[t]{2}{*}{180} & \multirow[t]{2}{*}{ Deacetylveralosine } & S. spirale & Root & & [470] \\
\hline & & S. diphyllum & Root & & [126] \\
\hline 181 & Solaspiralidine & S. spirale & Root & & {$[470]$} \\
\hline 182 & Soladunalinidine & S. arboreum & Aerial & & [59] \\
\hline 183 & 3-epi-Soladunalinidine & S. arboreum & Aerial & & [59] \\
\hline 184 & Caavuranamide & S. caavurana & Fruit & Antibacterial & {$[80]$} \\
\hline 185 & 4-Tomatiden-3-one & S. caavurana & Fruit & & {$[80]$} \\
\hline 186 & 5-Tomatidan-3-one & S. caavurana & Fruit & & {$[80]$} \\
\hline 187 & $(22 \mathrm{~S}, 25 \mathrm{~S})-3 \beta$-aminospirosol-5-ene & S. arboreum & Aerial & & [59] \\
\hline 188 & $(22 R, 25 R) 3 \beta$-amino- $5 \alpha$-spirosolane & S. triste & Aerial & & {$[362,471]$} \\
\hline 189 & $(22 R, 25 R) 3 \beta$-amino-5-spirosolene & S. triste & Aerial & & {$[362,471]$} \\
\hline 190 & Isojuripidine & S. asterophorum & Aerial & Spasmolytic & [70] \\
\hline 191 & $\begin{array}{l}\text { 23,24-2-methyl-tetrahydrofuran)Solani- } \\
\text { dine }\end{array}$ & S. cornifolium & Aerial & & {$[472,473]$} \\
\hline 192 & Spiraloside C & S. spirale & Fruit & & {$[474]$} \\
\hline 193 & Spiraloside B & S. spirale & Fruit & & [474] \\
\hline 194 & Spiraloside A & S. spirale & Fruit & & [474] \\
\hline
\end{tabular}


Table 2 (continued)

\begin{tabular}{|c|c|c|c|c|c|}
\hline No. & Compounds & Plant sources & Parts & Biological properties & References \\
\hline 195 & Soladulcine A & S. dulcamara & Aerial & & [433] \\
\hline 196 & Soladulcine B & S. dulcamara & Aerial & & [433] \\
\hline \multirow[t]{2}{*}{197} & Esculeoside A & S. lycopersicum & Fruit & & {$[475]$} \\
\hline & Pregnane glycosides & & & & \\
\hline 198 & Solanigroside A & S. nigrum & Whole & & {$[476]$} \\
\hline 199 & Solanigroside B & S. nigrum & Whole & & {$[476]$} \\
\hline 200 & $\begin{array}{l}5 \alpha \text {-Pregn-16-en-3 } \beta \text {-ol-20-one lycotetrao- } \\
\text { side }\end{array}$ & S. nigrum & Whole & & {$[476]$} \\
\hline 201 & (5 $\alpha$ )-3-Hydroxypregn-16-en-20-one & S. lyratum & Whole & & [194] \\
\hline \multirow[t]{2}{*}{202} & Hypoglaucin $\mathrm{H}$ & S. nigrum & Whole & & {$[476]$} \\
\hline & & S. rostratum & Aerial & & [437] \\
\hline 203 & 16-Dehydropregnolone & S. lyratum & Whole & Anticancer & [194] \\
\hline 204 & $\begin{array}{l}\text { 16-dehydropregnenolone } 3-O-\alpha \text {-L- } \\
\text { rhamnosyl-1-2) } \beta \text {-D-glucosiduronic acid }\end{array}$ & S. lyratum & Whole & & [194] \\
\hline 205 & Torvpregnanoside A & S. torvum & Aerial & & {$[317,331]$} \\
\hline 206 & $\begin{array}{l}5 \alpha \text {-pregn-16-en-3,20-dione- } 6 \alpha \text {-ol-6- } O \text { - }[\alpha \text { - } \\
\text { L-rhamnosyl-(1-3)- } \beta \text {-D-quinovoside] }\end{array}$ & S. torvum & Fruit & Anticancer & [317] \\
\hline 207 & Torvpregnanoside B & S. torvum & Aerial & & {$[331]$} \\
\hline 208 & Ganaxolone & S. torvum & Aerial & & {$[323]$} \\
\hline 209 & Allopregnanolone & S. torvum & Aerial & & [323] \\
\hline \multirow[t]{2}{*}{210} & Pregnanolone & S. torvum & Aerial & & [323] \\
\hline & Triterpenes & & & & \\
\hline 211 & Betulinic acid & S. buddleifolium & Stem & & [79] \\
\hline \multirow[t]{3}{*}{212} & Lupeol & S. cathayanum & Aerial & & {$[472,473,477]$} \\
\hline & & S. schimperianum & Aerial & & {$[278]$} \\
\hline & & S. spirale & Leaf & Anticancer & [297] \\
\hline 213 & Cycloeucalenone & S. сегnиит & Leaf & Anticancer & {$[107]$} \\
\hline 214 & 24-oxo-31-norcycloartanone & S. cеrnuит & Leaf & Anticancer & {$[107]$} \\
\hline 215 & Friedelin & S. lycopersicum & Seed & & {$[478]$} \\
\hline \multirow[t]{3}{*}{216} & Ursolic acid & S. lyratum & Whole & & [197] \\
\hline & & S. torvum & Aerial & & [463] \\
\hline & & S. xanthocarpum & Root & & [427] \\
\hline 217 & $2 \alpha, 3 \beta$-Dihydroxyursolic acid & S. torvum & Aerial & & [463] \\
\hline 218 & Daturaolone & S. arundo & Whole & & {$[65]$} \\
\hline 219 & Carbenoxolone & S. сегnиит & Leaf & & [109] \\
\hline 220 & $\beta$-Amyrin & S. melongena & Aerial & & [439] \\
\hline \multirow[t]{2}{*}{221} & Oleanolic acid & S. torvum & Aerial & & [463] \\
\hline & & S. xanthocarpum & Root & & {$[427]$} \\
\hline 222 & $2 \alpha$-Hydroxyoleanolic acid & S. torvum & Aerial & & {$[463]$} \\
\hline 223 & 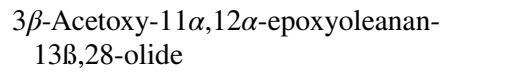 & S. torvum & Aerial & & {$[463]$} \\
\hline \multirow[t]{2}{*}{224} & Solanoglycosydane I & S. torvum & Fruit & & [314] \\
\hline & Diterpenes & & & & \\
\hline \multirow[t]{2}{*}{225} & Phytol & $\begin{array}{l}\text { S. pseudocapsi- } \\
\text { cum }\end{array}$ & Leaf & & [263] \\
\hline & & S. villosum & Leaf & & {$[434,479]$} \\
\hline 226 & Kaur-16-ene & S. aculeastrum & Leaf & & [11] \\
\hline 227 & Solanerioside A & S. erianthum & Leaf & & [138] \\
\hline \multirow[t]{2}{*}{228} & Tricalysioside U & S. violaceum & Whole & & [392] \\
\hline & Sesquiterpenes & & & & \\
\hline 229 & Roseoside & S. erianthum & Leaf & & [138] \\
\hline
\end{tabular}


Table 2 (continued)

\begin{tabular}{|c|c|c|c|c|c|}
\hline No. & Compounds & Plant sources & Parts & Biological properties & References \\
\hline 230 & $\begin{array}{l}(6 E, 10 E)-5,12 \text {-Dihydroxy- } \beta \text {-nerolidol } \\
5-O-\beta \text {-D-glucoside }\end{array}$ & S. erianthum & Leaf & & [138] \\
\hline 231 & Amarantholidoside IV & S. erianthum & Leaf & & [138] \\
\hline \multirow[t]{2}{*}{232} & $3 \beta$-Hydroxysolavetivone & S. abutiloides & Root & Antifungal & [3] \\
\hline & & S. aethiopicum & Root & & [29] \\
\hline \multirow[t]{4}{*}{233} & Solavetivone & S. abutiloides & Root & Antifungal & [3] \\
\hline & & S. aethiopicum & Root & & [29] \\
\hline & & S. indicum & Root & & {$[163]$} \\
\hline & & S. jabrense & Aerial & & [166] \\
\hline \multirow[t]{2}{*}{234} & 13-Hydroxysolavetivone & S. buddleifolium & Stem & & [79] \\
\hline & & S. aethiopicum & Root & & [29] \\
\hline \multirow[t]{2}{*}{235} & Lubimin & S. abutiloides & Root & Antifungal & [3] \\
\hline & & S. aethiopicum & Root & & [29] \\
\hline 236 & Lubiminoic acid & S. aethiopicum & Root & & [29] \\
\hline 237 & Epilubimin & S. aethiopicum & Root & & [29] \\
\hline 238 & Epilubiminoic acid & S. aethiopicum & Root & & [29] \\
\hline 239 & Lubiminol & S. aethiopicum & Root & & [29] \\
\hline 240 & $\alpha$-Farnesene & S. aculeastrum & Leaf & & {$[11]$} \\
\hline 241 & Nerolidol & S. aculeastrum & Leaf & & {$[11]$} \\
\hline 242 & 2,7,10-Trimethyldodecane & S. aculeastrum & Leaf & & {$[11]$} \\
\hline 243 & Aethione & S. aethiopicum & Root & & [29] \\
\hline 244 & Anhydro- $\beta$-rotunol & S. aethiopicum & Root & & [29] \\
\hline 245 & $\begin{array}{l}(4 S, 5 R, 7 S)-4,11 \text {-Dihydroxy-guaia- } \\
\text { 1(2),9(10)-dien }\end{array}$ & S. erianthum & Stem & & [480] \\
\hline 246 & Caryophyllene & S. erianthum & Fruit & & [481] \\
\hline 247 & Cadina-1(10),4-diene & S. erianthum & Fruit & & [481] \\
\hline 248 & $\alpha$-Gurjunene & S. erianthum & Fruit & & [481] \\
\hline 249 & Globulol & S. erianthum & Fruit & & [481] \\
\hline 250 & $\alpha$-Guaiene & S. erianthum & Fruit & & [481] \\
\hline 251 & $\alpha$-Calacorene & S. erianthum & Fruit & & [481] \\
\hline 252 & 2-naphthalenemethanol & S. erianthum & Fruit & & [481] \\
\hline 253 & $\begin{array}{l}\text { Octahydro-2,2-dimethyl-4a,7a-ethano- } \\
5 H \text {-cyclobut }[e] \text { inden-5-ol }\end{array}$ & S. erianthum & Fruit & & [481] \\
\hline 254 & 4,5-Dehydroisolongifolene & S. erianthum & Fruit & & [481] \\
\hline 255 & $\alpha$-Caryophyllene & S. erianthum & Fruit & & [481] \\
\hline 256 & Solafuranone & S. indicum & Root & & [163] \\
\hline \multirow[t]{2}{*}{257} & Lyratol D & S. lyratum & Whole & Anticancer & [199] \\
\hline & & S. septemlobum & Whole & & [482] \\
\hline \multirow[t]{2}{*}{258} & Solajiangxin B & S. lyratum & Whole & Anticancer & [198] \\
\hline & & S. septemlobum & Whole & & [482] \\
\hline 259 & Septemlobin D & S. septemlobum & Whole & & [483] \\
\hline 260 & Blumenol A & S. lyratum & Whole & Anticancer & {$[199,484]$} \\
\hline 261 & Blumenol C & S. lyratum & Whole & & [484] \\
\hline 262 & Dehydrovomifoliol & S. lyratum & Whole & Anticancer & {$[199,484]$} \\
\hline 263 & Grasshopper ketone & S. lyratum & Whole & & [484] \\
\hline 264 & $6 \alpha$-Epoxy-7-megastigmen-9-one & S. lyratum & Whole & & [484] \\
\hline 265 & $\begin{array}{l}\left(1^{\prime} R, 2 R, 5 S, 10 R\right) 2-1^{\prime}, 2^{\prime} \text {-dihydroxy-1'- } \\
\text { methylethyl)6,10-dimethylspiro[4, 5] } \\
\text { dec-6-en-8-one }\end{array}$ & S. lyratum & Whole & & [484] \\
\hline
\end{tabular}


Table 2 (continued)

\begin{tabular}{|c|c|c|c|c|c|}
\hline No. & Compounds & Plant sources & Parts & Biological properties & References \\
\hline 266 & $\begin{array}{l}\left(1^{\prime} S, 2 R, 5 S, 10 R\right) 2-1^{\prime}, 2^{\prime} \text {-dihydroxy-1'- } \\
\text { methylethyl)6,10-dimethylspiro[4, 5] } \\
\text { dec-6-en-8-one }\end{array}$ & S. lyratum & Whole & & [484] \\
\hline 267 & $\begin{array}{l}\text { 2-1',2'-dihydroxy-1'-methylethyl)6,10- } \\
\text { dimethyl-9-hydroxyspiro[4, 5]dec-6-en- } \\
\text { 8-one }\end{array}$ & S. lyratum & Whole & & {$[200,484]$} \\
\hline 268 & Boscialin & S. lyratum & Whole & & [484] \\
\hline 269 & $1 \beta$-Hydroxy-1,2-dihydro- $\alpha$-santonin & S. lyratum & Whole & & {$[193,484]$} \\
\hline 270 & Lyratol A & S. lyratum & Whole & & [485] \\
\hline \multirow[t]{2}{*}{271} & Lyratol B & S. lyratum & Whole & & {$[485]$} \\
\hline & & S. septemlobum & Whole & & {$[482]$} \\
\hline 272 & Lyratol C & S. lyratum & Whole & Anticancer & [199] \\
\hline 273 & Lyratol G & S. lyratum & Whole & & [196] \\
\hline 274 & Solajiangxin A & S. lyratum & Whole & Anticancer & [198] \\
\hline 275 & Solajiangxin C & S. lyratum & Whole & Anticancer & [198] \\
\hline \multirow[t]{2}{*}{276} & Solajiangxin D & S. lyratum & Whole & Anticancer & [200] \\
\hline & & S. septemlobum & Whole & & [482] \\
\hline 277 & Solajiangxin E & S. lyratum & Whole & Anticancer & [200] \\
\hline \multirow[t]{2}{*}{278} & Solajiangxin F & S. lyratum & Whole & Anticancer & [197] \\
\hline & & S. septemlobum & Whole & & {$[482]$} \\
\hline 279 & Solajiangxin G & S. lyratum & Whole & Anticancer & [197] \\
\hline 280 & 2-hydroxysolajiangxin E & S. lyratum & Whole & Anticancer & {$[200]$} \\
\hline \multirow[t]{2}{*}{281} & Dehydrocarissone & S. lyratum & Stem & & [486] \\
\hline & & S. septemlobum & Whole & & [482] \\
\hline 282 & Atractylenolide I & S. lyratum & Stem & & [486] \\
\hline 283 & Ligucyperonol & S. septemlobum & Whole & & [482] \\
\hline 284 & Nardoeudesmol A & S. septemlobum & Whole & & [482] \\
\hline 285 & Solanerianone A & S. septemlobum & Whole & & [482] \\
\hline 286 & Pterocarptriol & S. torvum & Root & & [487] \\
\hline 287 & Selina- $3 \beta, 4 \alpha, 11$-triol & S. torvum & Root & & [487] \\
\hline 288 & $\begin{array}{l}\text { 2-(1',2'-dihydroxy-1'-methylethyl)-6,10- } \\
\text { dimethylspiro[4, 5]dec-6,9-dien-8-one }\end{array}$ & S. torvum & Root & & [487] \\
\hline 289 & $10 \beta, 12,14$-Trihydroxy-allo-aromadendrane & S. torvum & Root & & [487] \\
\hline 290 & $10 \beta, 13,14$-Trihydroxy-allo-aromadendrane & S. torvum & Root & & [487] \\
\hline 291 & $\begin{array}{l}\text { 2-(1',2'-dihydroxy-1'-methylethyl)-6,10- } \\
\text { dimethyl-9-hydroxy-spirodec-6-en-8-one }\end{array}$ & S. torvum & Root & & [487] \\
\hline 292 & $\begin{array}{l}1 \beta, 10 \beta, 12,14 \text {-Tetrahydroxy-allo- } \\
\text { aromadendrane }\end{array}$ & S. torvum & Root & & [487] \\
\hline 293 & $\begin{array}{l}1 \beta, 10 \beta, 13,14-\text { Tetrahydroxy-allo- } \\
\text { aromadendrane }\end{array}$ & S. torvum & Root & & [487] \\
\hline 294 & Teferidin & S. schimperianum & Aerial & & {$[278]$} \\
\hline 295 & Teferin & S. schimperianum & Aerial & & {$[278]$} \\
\hline 296 & Ferutinin & S. schimperianum & Aerial & & [278] \\
\hline 297 & Bisabolol & S. sessiliflorum & Fruit & & [488] \\
\hline 298 & 11,12 - $O$-Isopropylidenesolajiangxin $\mathrm{F}$ & S. septemlobum & Whole & & [483] \\
\hline 299 & Eudesmane & S. septemlobum & Whole & & [281] \\
\hline 300 & Vitispirane & S. septemlobum & Whole & & [281] \\
\hline 301 & Septemlobin A & S. septemlobum & Whole & Anticancer & [281] \\
\hline 302 & Septemlobin B & S. septemlobum & Whole & Anticancer & [281] \\
\hline 303 & Septemlobin C & S. septemlobum & Whole & Anticancer & {$[281]$} \\
\hline
\end{tabular}


Table 2 (continued)

\begin{tabular}{|c|c|c|c|c|c|}
\hline No. & Compounds & Plant sources & Parts & Biological properties & References \\
\hline 304 & $\begin{array}{l}3 \beta, 11 \text {-dihydroxy- } 4,14- \\
\text { oxideenantioeudesmane }\end{array}$ & S. torvum & Root & & [487] \\
\hline 305 & Aromadendrene oxide & S. erianthum & Fruit & & [481] \\
\hline 306 & Thujopsene & S. betaceum & Fruit & & [77] \\
\hline 307 & $\alpha$-Cedrene & S. betaceum & Fruit & & [77] \\
\hline 308 & Cedrol & S. betaceum & Fruit & & [77] \\
\hline 309 & $\alpha$-Hexylcinnamaldehyde & S. betaceum & Fruit & & [77] \\
\hline \multirow[t]{2}{*}{310} & $\beta$-Cadinene & S. betaceum & Fruit & & [77] \\
\hline & Monoterpenes & & & & \\
\hline 311 & Decanal & S. aculeastrum & Leaf & & [11] \\
\hline 312 & Decane & S. aculeastrum & Leaf & & [11] \\
\hline 313 & 2,4-Decadienal & S. aculeastrum & Leaf & & [11] \\
\hline 314 & 1,8-Cineole & S. betaceum & Fruit & & [77] \\
\hline 315 & Terpinen-4-ol & S. betaceum & Fruit & & [77] \\
\hline 316 & Linalool & S. vestissimum & Fruit & & {$[489,490]$} \\
\hline 317 & Geraniol & S. vestissimum & Fruit & & [490] \\
\hline 318 & Limonene & S. vestissimum & Fruit & & [490] \\
\hline 319 & $\beta$-Cyclocitral & S. aculeastrum & Leaf & & [11] \\
\hline \multirow[t]{3}{*}{320} & $\beta$-Ionone & S. aculeastrum & Leaf & & [11] \\
\hline & & $\begin{array}{l}\text { S. pseudocapsi- } \\
\text { cum }\end{array}$ & Leaf & & [263] \\
\hline & & S. betaceum & Fruit & & [77] \\
\hline 321 & 1, 2-Dihydro-1,1,6-trimethyl-naphthalene & S. aculeastrum & Leaf & & {$[11]$} \\
\hline 322 & trans- $\beta$-Damascenone & S. aculeastrum & Leaf & & [11] \\
\hline \multirow[t]{3}{*}{323} & Loliolide & S. erianthum & Leaf & & [137] \\
\hline & & S. americanum & Aerial & & [49] \\
\hline & & $\begin{array}{l}\text { S. pseudocapsi- } \\
\text { cum }\end{array}$ & Leaf & & {$[263]$} \\
\hline 324 & Hotrienol & S. vestissimum & Fruit & & {$[468,490]$} \\
\hline 325 & Neroloxide & S. vestissimum & Fruit & & [468] \\
\hline 326 & $\begin{array}{l}\text { 5-Ethynyltetrahydro- } \alpha, \alpha, 5 \text {-trimethyl-2- } \\
\text { furanmethanol }\end{array}$ & S. vestissimum & Fruit & & [490] \\
\hline 327 & Nerol & S. vestissimum & Fruit & & [490] \\
\hline 328 & 8-Hydroxylinalool & S. vestissimum & Fruit & & [491] \\
\hline 329 & $(R)$-Linalyl $\beta$-D-glucoside & S. vestissimum & Fruit & & [492] \\
\hline 330 & $\begin{array}{l}\text { (1R,4E)-1-Ethenyl-6-hydroxy-1,5-dime- } \\
\text { thyl-4-hexen-1-yl } \beta \text {-D-glucoside }\end{array}$ & S. vestissimum & Fruit & & [492] \\
\hline 331 & $(R)$-Linalyl $\beta$-vicianoside & S. vestissimum & Fruit & & [492] \\
\hline 332 & $\begin{array}{l}\text { 6- } O \text {-linked } \beta \text {-D-glucoside of }(R) E) 2,6- \\
\text { dimethyl-3,7-octadiene-2,6-diol }\end{array}$ & S. vestissimum & Fruit & & {$[468]$} \\
\hline 333 & $\begin{array}{l}\text { (3E,6R)-2,6-Dimethyl-3,7-octadiene- } \\
\text { 2,6-diol }\end{array}$ & S. vestissimum & Fruit & & {$[468]$} \\
\hline 334 & $p$-Cymenene & S. betaceum & Fruit & & [77] \\
\hline 335 & Dihydroactinidiolide & S. erianthum & Leaf & & [137] \\
\hline 336 & Apiole & S. sessiliflorum & Fruit & & [488] \\
\hline 337 & $\alpha$-Terpinen-7-al & S. betaceum & Fruit & & [77] \\
\hline \multirow[t]{2}{*}{338} & 1,3,8-p-Menthatriene & S. betaceum & Fruit & & [77] \\
\hline & Flavonoids & & & & \\
\hline 339 & Vitecetin & S. agrarium & Aerial & & [31] \\
\hline
\end{tabular}


Table 2 (continued)

\begin{tabular}{|c|c|c|c|c|c|}
\hline No. & Compounds & Plant sources & Parts & Biological properties & References \\
\hline \multirow[t]{7}{*}{340} & \multirow[t]{7}{*}{ Quercetin } & S. anguvi & Fruit & \multirow[t]{8}{*}{ Anticancer } & [31] \\
\hline & & S. elaeagnifolium & Seed & & [493] \\
\hline & & S. incanum & Aerial & & [494] \\
\hline & & S. melongena & Stem & & [205] \\
\hline & & S. muricatum & Whole & & [215] \\
\hline & & S. nigrum & Leaf & & $\begin{array}{l}{[92-98,230-238,} \\
495-497]\end{array}$ \\
\hline & & S. torvum & Whole & & [498] \\
\hline 341 & Kaempferol 7- $O$-rhamnoside & S. asperum & Fruit & & {$[67]$} \\
\hline \multirow[t]{5}{*}{342} & \multirow[t]{5}{*}{ Rutin } & S. anguvi & Fruit & \multirow[t]{17}{*}{ Anticancer } & [31] \\
\hline & & S. melongena & Stem & & {$[499,500]$} \\
\hline & & S. muricatum & Fruit & & [215] \\
\hline & & S. nigrum & Leaf & & [230] \\
\hline & & S. spirales & Aerial & & {$[470]$} \\
\hline 343 & Kaempferol 3-rutinoside-7-rhamnoside & S. asperum & Fruit & & {$[67]$} \\
\hline 344 & Afzelin & S. сегпит & Leaf & & {$[109,112,501]$} \\
\hline \multirow[t]{2}{*}{345} & \multirow[t]{2}{*}{ Quercitrin } & S. cernuит & Leaf & & [109] \\
\hline & & S. melongena & Stem & & [205] \\
\hline \multirow[t]{4}{*}{346} & \multirow[t]{4}{*}{ Astragalin } & S. cernuит & Leaf & & [501] \\
\hline & & S. crinitum & Aerial & & [459] \\
\hline & & S. incanum & Aerial & & [494] \\
\hline & & S. elaeagnifolium & Aerial & & {$[502]$} \\
\hline 347 & $\begin{array}{l}\text { Kaempferol 3- } O \text {-[ } \alpha \text {-apiofuranosyl-(1-2)]- } \\
\alpha \text {-rhamnoside }\end{array}$ & S. сегпиит & Leaf & & {$[501]$} \\
\hline 348 & $\begin{array}{l}\text { Kaempferol 3-O-[ } \alpha \text {-apiofuranosyl-(1-2)]- } \\
\quad \beta \text {-galactoside }\end{array}$ & S. сегпиит & Leaf & & [501] \\
\hline \multirow[t]{4}{*}{349} & \multirow[t]{4}{*}{ Tiliroside } & S. asperum & Fruit & & {$[67]$} \\
\hline & & S. crinitum & Aerial & & {$[123,459]$} \\
\hline & & S. elaeagnifolium & Whole & Anticancer & {$[503]$} \\
\hline & & S. cеrnuит & Leaf & & [501] \\
\hline \multirow[t]{2}{*}{350} & \multirow[t]{2}{*}{ cis-Tiliroside } & S. сегпиит & Leaf & & [501] \\
\hline & & S. elaeagnifolium & Aerial & & {$[502]$} \\
\hline \multirow[t]{7}{*}{351} & \multirow[t]{7}{*}{ Kaempferol } & S. crinitum & Aerial & & [459] \\
\hline & & S. elaeagnifolium & Whole & & [504] \\
\hline & & S. incanum & Aerial & & [494] \\
\hline & & S. indicum & Whole & & [505] \\
\hline & & S. nigrum & Leaf & & [227] \\
\hline & & S. surattense & Whole & & [99] \\
\hline & & S. torvum & Whole & & [498] \\
\hline 352 & Camelliaside C & S. erianthum & Leaf & & [137] \\
\hline 353 & Baimaside & S. incanum & Aerial & & [506] \\
\hline 354 & Narcissin & S. glabratum & Aerial & & [141] \\
\hline 355 & Isorhamnetin 3-glucoside & S. incanum & Aerial & & [506] \\
\hline 356 & Populnin & S. elaeagnifolium & Aerial & & [502] \\
\hline 357 & Quercetin 3-O-robinoside & S. paniculatum & Aerial & & [258] \\
\hline 358 & $\begin{array}{l}\text { Kaempferol 3-O-(6"-O-cis- } p \text {-coumaroyl)- } \\
O \text { - } \beta \text {-galactoside }\end{array}$ & S. elaeagnifolium & Aerial & & [502] \\
\hline 359 & Myricetin-3-galactoside & S. melongena & Stem & & [205] \\
\hline \multirow[t]{2}{*}{360} & \multirow[t]{2}{*}{ Apigenin } & S. lyratum & Whole & & [507] \\
\hline & & S. torvum & Whole & & [498] \\
\hline
\end{tabular}


Table 2 (continued)

\begin{tabular}{|c|c|c|c|c|c|}
\hline No. & Compounds & Plant sources & Parts & Biological properties & References \\
\hline 361 & Pelanin & S. tuberosum & Stem & & {$[508]$} \\
\hline 362 & Petanin & S. tuberosum & Stem & & {$[508]$} \\
\hline 363 & Peonanin & S. tuberosum & Stem & & [508] \\
\hline 364 & Keracyanin & S. betaceum & Fruit & Anticancer & {$[75,76]$} \\
\hline 365 & Pelargonidin 3-rutinoside & S. betaceum & Fruit & Anticancer & {$[75,76]$} \\
\hline 366 & Tulipanin & S. betaceum & Fruit & Anticancer & {$[75,76]$} \\
\hline 367 & $\begin{array}{l}\text { Delphinidin 3- } O-\alpha \text {-L-rhamnosyl-(1-6)- } \beta \text {-D- } \\
\text { glucoside-3'- } O-\beta \text {-D-glucoside }\end{array}$ & S. betaceum & Fruit & Anticancer & {$[75,76]$} \\
\hline 368 & Cyanidin 3-O-(2"-O-xylosyl)rutinoside & S. betaceum & Fruit & & [76] \\
\hline 369 & Asterin & S. betaceum & Fruit & & [76] \\
\hline 370 & $\begin{array}{l}\text { Biochanin A-7- } O-\beta \text {-D-apiofuranosyl-1- } \\
\text { 5) } \beta \text {-D-apiofuranosyl-1-6) } \beta \text {-D-glucoside }\end{array}$ & S. crinitum & Fruit & & [122] \\
\hline 371 & $\begin{array}{l}\text { 2R,3R-5,7,4'-trihydroxy-dihydroflavon-3- } \\
O \text { - } \alpha \text {-D-glucosyl-6"- } O \text { - } \beta \text {-D-glucoside- } 6^{\prime \prime \prime} \text { - } \\
\text { p-hydroxy benzoate }\end{array}$ & S. elaeagnifolium & Whole & Anticancer & {$[503]$} \\
\hline 372 & $6,2^{\prime}, 3^{\prime \prime}, 5^{\prime \prime}, 4^{\prime \prime \prime}$-Pentahydroxy-3,7"-biflavone & S. dulcamara & Fruit & & {$[130]$} \\
\hline 373 & Kaempferol 8- $C$ - $\beta$-D-galactoside & S. elaeagnifolium & Aerial & Hepatoprotective & [502] \\
\hline 374 & Kaempferol 8-C-glucoside & S. elaeagnifolium & Aerial & & [502] \\
\hline 375 & Kaempferol 6- $C$-glucoside & S. elaeagnifolium & Aerial & & [502] \\
\hline 376 & Vitexin & S. elaeagnifolium & Aerial & & [502] \\
\hline 377 & Vicenin II & S. elaeagnifolium & Aerial & & [502] \\
\hline 378 & Quercetin 6-C- $\beta$-glucoside & S. elaeagnifolium & Aerial & & {$[502]$} \\
\hline 379 & Quercetin 3-O- $\beta$-galactoside & S. elaeagnifolium & Aerial & & [502] \\
\hline \multirow[t]{4}{*}{380} & Isoquercitrin & S. elaeagnifolium & Aerial & & {$[502-504]$} \\
\hline & & S. incanum & Aerial & & [494] \\
\hline & & S. torvum & Root & & [338] \\
\hline & & S. melongena & Stem & & [205] \\
\hline 381 & $\begin{array}{l}\text { Quercetin 3-O- } \beta \text {-apiofuranosyl-(1-2)-O- } \beta \text { - } \\
\text { galactoside }\end{array}$ & S. elaeagnifolium & Aerial & & {$[502]$} \\
\hline 382 & 5-Hydroxy, 7,2',3',5'-tetramethoxyflavone & S. glabratum & Whole & & [140] \\
\hline 383 & Combretol & S. glabratum & Whole & & [140] \\
\hline 384 & Baicalin & S. incanum & Aerial & & [506] \\
\hline 385 & $\begin{array}{l}\text { Kaempferol 3-O- }\left(6^{\prime \prime \prime}-O-2,5-\right. \\
\text { dihydroxycinnamoyl)- } \beta \text {-D-glucosyl(1-2) } \\
\beta \text {-D-glucoside }\end{array}$ & S. incanum & Aerial & & [506] \\
\hline \multirow[t]{4}{*}{386} & $( \pm)$-Naringenin & S. indicum & Whole & & {$[505]$} \\
\hline & & S. nienkui & Whole & & [509] \\
\hline & & S. sessiliflorum & Fruit & & [510] \\
\hline & & S. surattense & Whole & & [99] \\
\hline 387 & Manghaslin & S. lycopersicum & Fruit & & [511] \\
\hline \multirow[t]{2}{*}{388} & Genkwanin & S. jabrense & Aerial & & [167] \\
\hline & & S. palodusum & Aerial & & [512] \\
\hline 389 & Ombuine & S. jabrense & Aerial & & [167] \\
\hline \multirow[t]{2}{*}{390} & Rhamnocitrin & S. jabrense & Aerial & & [167] \\
\hline & & S. palodusum & Aerial & & [513] \\
\hline \multirow[t]{4}{*}{391} & Retusin & S. jabrense & Aerial & & [167] \\
\hline & & S. palodusum & Aerial & & [512] \\
\hline & & S. schimperianum & Aerial & & [278] \\
\hline & & S. torvum & Fruit & & [322] \\
\hline 392 & Pentamethoxyquercetin & S. jabrense & Aerial & & [167] \\
\hline
\end{tabular}


Table 2 (continued)

\begin{tabular}{|c|c|c|c|c|c|}
\hline No. & Compounds & Plant sources & Parts & Biological properties & References \\
\hline \multirow[t]{2}{*}{393} & \multirow[t]{2}{*}{ 3-O-Methylquercetin } & S. jabrense & Aerial & & [167] \\
\hline & & S. palodusum & Aerial & & [513] \\
\hline \multirow{2}{*}{394} & \multirow{2}{*}{ Kumatakenin } & S. jabrense & Aerial & & {$[167]$} \\
\hline & & S. palodusum & Aerial & & [513] \\
\hline \multirow[t]{2}{*}{395} & \multirow[t]{2}{*}{ 3'-Hydroxyflindulatin } & S. jabrense & Aerial & & [167] \\
\hline & & S. palodusum & Aerial & & {$[513]$} \\
\hline 396 & 3,7,8-Trimethylherbacetin & S. jabrense & Aerial & & [167] \\
\hline \multirow[t]{2}{*}{397} & \multirow[t]{2}{*}{ 3,7,8,3',4'-Pentamethylgossypetin } & S. jabrense & Aerial & & [167] \\
\hline & & S. palodusum & Aerial & & {$[512,513]$} \\
\hline 398 & Diosmetin & S. nienkui & Whole & & [509] \\
\hline 399 & Formononetin & S. lyratum & Whole & & [514] \\
\hline 400 & Ononin & S. lyratum & Whole & & [514] \\
\hline 401 & Daidzein & S. lyratum & Whole & & {$[507,514]$} \\
\hline 402 & Genistin & S. lyratum & Whole & & [514] \\
\hline 403 & 5-Hydroxylononin & S. lyratum & Whole & & [514] \\
\hline 404 & $\begin{array}{l}\text { 2,7-Dihydroxy-3-(4-hydroxyphenyl)- } \\
\text { 5-methoxy-4H-1-benzopyran-4-one }\end{array}$ & S. nienkui & Whole & & [509] \\
\hline 405 & 5-hydroxy-3,7,4'-trimethoxyflavone & S. schimperianum & Aerial & & [278] \\
\hline 406 & Kaempferol-3- $O-\beta$-D-glucoside & S. schimperianum & Aerial & & [278] \\
\hline 407 & Luteolin & S. schimperianum & Aerial & & {$[278]$} \\
\hline 408 & Tamarixin & S. torvum & Whole & & [498] \\
\hline 409 & Torvanol A & S. torvum & Root & Antidepressant, antiviral & {$[322,332]$} \\
\hline 410 & $\begin{array}{l}\text { 5-methoxy-(3,4"-dihydro-3",4"- } \\
\text { diacetoxy)-2", } 2^{\prime} \text {-dimethyl-(7,8:5",6")- } \\
\text { flavone }\end{array}$ & S. erianthum & Leaf & & [137] \\
\hline 411 & $\begin{array}{l}\text { 5,7,8,4'-tetrahydroxy-3-methoxyflavone- } 8 \text { - } \\
O \text { - } \beta \text {-D-xyloside }\end{array}$ & S. rostratum & Aerial & & {$[515]$} \\
\hline 412 & 3-O-Methylquercetin 3-O- $\beta$-D-galactoside & S. rostratum & Whole & & [516] \\
\hline \multirow[t]{2}{*}{413} & 3-O-Methylquercetin 3-O- $\beta$-D-glucoside & S. rostratum & Whole & & [516] \\
\hline & Lignans & & & & \\
\hline 414 & Isolariciresinol & S. buddleifolium & Stem & & [79] \\
\hline 415 & 5-Methoxyisolariciresinol & S. buddleifolium & Stem & & [79] \\
\hline 416 & Polystachyol & S. buddleifolium & Stem & & [79] \\
\hline 417 & (+)-Lyoniresinol 3-O-D-glucoside & S. buddleifolium & Stem & & [79] \\
\hline 418 & (-)-Lyoniresinol 3-O-D-glucoside & S. buddleifolium & Stem & & [79] \\
\hline 419 & Alangilignoside $\mathrm{C}$ & S. buddleifolium & Stem & & [79] \\
\hline 420 & $\begin{array}{l}(+)-\left(7 S, 8 R, 7^{\prime} E\right)-4 \text {-Hydroxy-3,5,5', } 9^{\prime} \text { - } \\
\text { tetram ethoxy-4',7-epoxy-8,3'-neo-lign- } \\
\text { 7'-en-9-ol }\end{array}$ & S. erianthum & Stem & & [480] \\
\hline 421 & $\begin{array}{l}\text { (-)-( }\left(7 R, 8 S, 7^{\prime} E\right)-4 \text {-Hydroxy-3,5,5',9'- } \\
\text { tetramethoxy-4',7-epoxy-8,3'-neo-lign- } \\
\text { 7'-en-9-ol }\end{array}$ & S. erianthum & Stem & & {$[480]$} \\
\hline 422 & Liriodendrin & S. lyratum & Whole & & [517] \\
\hline \multirow[t]{3}{*}{423} & \multirow[t]{3}{*}{ Syringaresinol } & S. lyratum & Whole & & [517] \\
\hline & & S. nigrum & Whole & & [496] \\
\hline & & S. surattense & Whole & & [518] \\
\hline 424 & Melongenamide A & S. melongena & Root & & [210] \\
\hline 425 & Cannabisin D & S. melongena & Root & Anti-inflammatory & [210] \\
\hline 426 & Melongenamide B & S. melongena & Root & Anti-inflammatory & [210] \\
\hline 427 & Grossamide & S. melongena & Root & Anti-inflammatory & {$[210]$} \\
\hline 428 & Melongenamide C & S. melongena & Root & Anti-inflammatory & [210] \\
\hline
\end{tabular}


Table 2 (continued)

\begin{tabular}{|c|c|c|c|c|c|}
\hline No. & Compounds & Plant sources & Parts & Biological properties & References \\
\hline 429 & Cannabisin F & S. melongena & Root & Anti-inflammatory & [210] \\
\hline 430 & Melongenamide D & S. melongena & Root & Anti-inflammatory & {$[210]$} \\
\hline 431 & Cannabisin $\mathrm{G}$ & S. melongena & Root & Anti-inflammatory & [210] \\
\hline 432 & $\begin{array}{l}\text { 1,2-dihydro-6,8-dimethoxy-7-hydroxy- } \\
\text { 1-(3,5-dimethoxy-4-hydroxyphenyl)- } \\
N^{1}, N^{2} \text {-bis-[2-(4-hydroxyphenyl)ethyl]- } \\
\text { 2,3-naphthalene dicarboxamide }\end{array}$ & S. melongena & Root & & [210] \\
\hline 433 & Sisymbrifolin & S. sisymbriifolium & Fruit & & [519] \\
\hline 434 & Grossamide $\mathrm{K}$ & S. melongena & Root & & [210] \\
\hline 435 & Pinoresinol & S. nigrum & Whole & & [496] \\
\hline 436 & Pinoresinol 4- $O-\beta$-D-glucoside & S. nigrum & Whole & & {$[520]$} \\
\hline \multirow[t]{2}{*}{437} & Medioresinol & S. nigrum & Whole & & [496] \\
\hline & & S. torvum & Stem & & [436] \\
\hline 438 & Syringaresinol-4'-O- $\beta$-D-glucoside & S. nigrum & Whole & & {$[520]$} \\
\hline 439 & Glycosmisic acid & S. surattense & Whole & & [518] \\
\hline 440 & Simulanol & S. surattense & Whole & & [518] \\
\hline 441 & Balanophonin & S. surattense & Whole & & [518] \\
\hline 442 & Ficusal & S. melongena & Root & & [209] \\
\hline 443 & Tribulusamide A & S. surattense & Whole & & [518] \\
\hline \multirow[t]{2}{*}{444} & Clemastanin B & S. torvum & Fruit & & {$[521]$} \\
\hline & Other alkaloids & & & & \\
\hline 445 & Xylogranatinine & S. cathayanum & Stem & & [477] \\
\hline 446 & Cernumidine & S. cernuum & Leaf & & {$[109,111,112]$} \\
\hline 447 & Isocernumidine & S. сегпиит & Leaf & & [111] \\
\hline 448 & Cernidine & S. сегпиит & Leaf & & {$[501]$} \\
\hline 449 & Ethyl orotate & S. cathayanum & Stem & & {$[103,477]$} \\
\hline 450 & 3-Indolecarboxylic acid & S. americanum & Aerial & & [49] \\
\hline 451 & L-Valyl-L-isoleucyl-L-leucine & S. asperum & Fruit & & [67] \\
\hline 452 & 2-Methyltetrahydro- $\beta$-carboline & S. jabrense & Aerial & & [166] \\
\hline 453 & Proline & S. asperum & Fruit & & [67] \\
\hline 454 & Acetamide & S. schimperianum & Aerial & & [277] \\
\hline 455 & Stearamide & S. schimperianum & Aerial & & [277] \\
\hline 456 & $\begin{array}{l}(6 E, 9 E) N, N \text {-dimethyloctadeca-6,9-dien- } \\
\text { amide }\end{array}$ & S. schimperianum & Aerial & & [277] \\
\hline 457 & $\begin{array}{l}\text { (2E)-3-(4-Hydroxyphenyl)- } \mathrm{N}-[(2 S)-2- \\
\text { (4-hydroxyphenyl)-2-methoxyethyl]-2- } \\
\text { propenamide }\end{array}$ & S. torvum & Aerial & & {$[450]$} \\
\hline \multirow[t]{7}{*}{458} & 4-Coumaroyltyramine & S. buddleifolium & Stem & & [79] \\
\hline & & S. cathayanum & Stem & & {$[522]$} \\
\hline & & S. indicum & Root & & {$[163]$} \\
\hline & & S. melongena & Root & & [209] \\
\hline & & S. surattense & Whole & & [518] \\
\hline & & S. torvum & Aerial & & [338] \\
\hline & & S. lyratum & Whole & & {$[507]$} \\
\hline \multirow[t]{5}{*}{459} & $N$-trans-Feruloyltyramine & S. buddleifolium & Stem & & [79] \\
\hline & & S. cathayanum & Stem & & {$[522]$} \\
\hline & & S. indicum & Root & & [163] \\
\hline & & S. melongena & Root & Antidiabetic & [209] \\
\hline & & S. lyratum & Whole & & [507] \\
\hline
\end{tabular}


Table 2 (continued)

\begin{tabular}{|c|c|c|c|c|c|}
\hline No. & Compounds & Plant sources & Parts & Biological properties & References \\
\hline \multirow[t]{2}{*}{460} & \multirow[t]{2}{*}{$N$-trans-Feruloylmethoxytyramine } & S. buddleifolium & Stem & & [79] \\
\hline & & S. cathayanum & Stem & & [522] \\
\hline 461 & $N$-trans-Caffeoyltyramine & S. buddleifolium & Stem & & [79] \\
\hline 462 & $N$-trans-Feruloyldopamine & S. buddleifolium & Stem & & [79] \\
\hline \multirow[t]{2}{*}{463} & \multirow[t]{2}{*}{$N$-trans-Feruloyloctopamine } & S. cathayanum & Stem & & {$[522]$} \\
\hline & & S. septemlobum & Aerial & & [523] \\
\hline \multirow[t]{2}{*}{464} & \multirow[t]{2}{*}{$N$-trans-p-coumaroyloctopamine } & S. americanum & Aerial & Antidiabetic & [49] \\
\hline & & S. torvum & Aerial & & {$[524]$} \\
\hline 465 & $N$-trans- $p$-feruloyloctopamine & S. americanum & Aerial & Antidiabetic & [49] \\
\hline \multirow[t]{2}{*}{466} & \multirow[t]{2}{*}{$N$-trans-p-coumaroyltyramine } & S. americanum & Aerial & Antidiabetic & [49] \\
\hline & & S. melongena & Root & & \\
\hline \multirow[t]{2}{*}{467} & \multirow[t]{2}{*}{$N$-trans- $p$-feruloytyramine } & S. americanum & Aerial & Antidiabetic & [49] \\
\hline & & S. torvum & Aerial & & {$[524]$} \\
\hline 468 & $N$-cis-p-Coumaroyltyramine & S. melongena & Root & & [209] \\
\hline 469 & Caffeoylputrescine & S. melongena & Stem & & [205] \\
\hline 470 & $\begin{array}{l}\text { 3-(3,4-Dihydroxyphenyl)- } N \text {-[3-[[4-[[3- } \\
\text { (3,4-dihydroxyphenyl)-1-oxo-2-propen- } \\
\text { 1-yl] amino]butyl]amino]propyl]-2-pro- } \\
\text { penamide }\end{array}$ & S. melongena & Stem & & [205] \\
\hline 471 & Aurantiamide acetate & S. torvum & Aerial & & {$[524]$} \\
\hline 472 & $N^{1}, N^{4}, N^{8}$-Tris(dihydrocaffeoyl) spermidine & S. sessiliflorum & Fruit & & {$[525]$} \\
\hline 473 & $\begin{array}{l}N \text {-(4-Aminobutyl)- } N \text {-[3-[[3-(3,4- } \\
\text { dihydroxyphenyl)-1-oxopropyl] amino] } \\
\text { propyl]-3,4-dihydroxybenzenepropan- } \\
\text { amide }\end{array}$ & S. sessiliflorum & Fruit & & {$[525]$} \\
\hline 474 & $\begin{array}{l}N \text {-(3-Aminopropyl)- } N \text {-[4-[[3-(3,4- } \\
\text { dihydroxyphenyl)-1-oxopropyl] amino] } \\
\text { butyl]-3,4-dihydroxybenzenepropana- } \\
\text { mide }\end{array}$ & S. sessiliflorum & Fruit & & {$[525]$} \\
\hline \multirow[t]{2}{*}{475} & Soya-cerebroside I & S. torvum & Root & & {$[435]$} \\
\hline & \multicolumn{5}{|l|}{ Sterols } \\
\hline 476 & Cilistol G & S. capsicoides & Leaf & & {$[85]$} \\
\hline 477 & Capsisteroid A & S. capsicoides & Leaf & & {$[85]$} \\
\hline 478 & Capsisteroid B & S. capsicoides & Leaf & & {$[85]$} \\
\hline 479 & Capsisteroid C & S. capsicoides & Leaf & & [85] \\
\hline 480 & Capsisteroid D & S. capsicoides & Leaf & & [85] \\
\hline 481 & Capsisteroid E & S. capsicoides & Leaf & & [85] \\
\hline 482 & Capsisteroid F & S. capsicoides & Leaf & & [85] \\
\hline \multirow[t]{13}{*}{483} & \multirow[t]{13}{*}{$\beta$-Sitosterol } & S. cathayanum & Stem & & {$[477,522]$} \\
\hline & & S. anguvi & Fruit & & {$[34]$} \\
\hline & & S. cornifolium & Aerial & & {$[472,473]$} \\
\hline & & S. dulcamara & Fruit & & [130] \\
\hline & & S. elaeagnifolium & Whole & & {$[134,504]$} \\
\hline & & S. indicum & Whole & & {$[160]$} \\
\hline & & S. lycopersicum & Seed & & [478] \\
\hline & & S. melongena & Aerial & & {$[206,439]$} \\
\hline & & S. schimperianum & Aerial & & {$[278]$} \\
\hline & & S. surattense & Aerial & & [518] \\
\hline & & S. torvum & Root & & {$[526]$} \\
\hline & & S. trilobatum & Whole & & [356] \\
\hline & & S. xanthocarpum & Fruit & & [398] \\
\hline
\end{tabular}


Table 2 (continued)

\begin{tabular}{|c|c|c|c|c|c|}
\hline No. & Compounds & Plant sources & Parts & Biological properties & References \\
\hline \multirow[t]{8}{*}{484} & Daucosterol & S. cathayanum & Stem & & {$[522]$} \\
\hline & & S. chrysotrichum & Leaf & & {$[120]$} \\
\hline & & S. elaeagnifolium & Whole & & [504] \\
\hline & & S. glabratum & Whole & & [140] \\
\hline & & S. ligustrinum & Aerial & & [179] \\
\hline & & S. septemlobum & Aerial & & [523] \\
\hline & & S. torvum & Root & & [526] \\
\hline & & S. violaceum & Whole & & [392] \\
\hline \multirow[t]{2}{*}{485} & Campesterol & S. elaeagnifolium & Seed & & [134] \\
\hline & & S. melongena & Root & & [439] \\
\hline \multirow[t]{2}{*}{486} & Cholesterol & S. lycopersicum & Seed & & [478] \\
\hline & & S. sessiliflorum & Fruit & & [285] \\
\hline 487 & $\gamma$-Sitosterol & S. lycopersicum & Seed & & [478] \\
\hline 488 & 7-Oxositosterol & S. violaceum & Aerial & & [391] \\
\hline 489 & 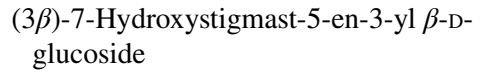 & S. violaceum & Whole & & [392] \\
\hline \multirow[t]{8}{*}{490} & Stigmasterol & S. cornifolium & Aerial & & {$[472,473]$} \\
\hline & & S. dulcamara & Fruit & & {$[130]$} \\
\hline & & S. elaeagnifolium & Whole & & {$[134,504]$} \\
\hline & & S. lycopersicum & Seed & & {$[478]$} \\
\hline & & S. melongena & Aerial & & [439] \\
\hline & & S. septemlobum & Aerial & & {$[523]$} \\
\hline & & S. surattense & Aerial & & [527] \\
\hline & & S. xanthocarpum & Fruit & & [398] \\
\hline 491 & Brassicasterol & S. elaeagnifolium & Seed & & [134] \\
\hline 492 & Poriferasterol monoglucoside & S. glabratum & Whole & & [140] \\
\hline 493 & 7-Oxostigmasterol & S. violaceum & Aerial & & [391] \\
\hline 494 & $\begin{array}{l}\beta \text {-stigmasteryl-3-O- } \beta \text {-D-6-palmityl) } \\
\text { glucoside }\end{array}$ & S. septemlobum & Aerial & & {$[523]$} \\
\hline 495 & Clerosterol & S. elaeagnifolium & Seed & & [134] \\
\hline 496 & 7-Sitoster-3 $\beta$-ol & S. elaeagnifolium & Seed & & [134] \\
\hline 497 & $(3 \beta, 5 \alpha)$ Cholest-7-en-3-ol & S. lycopersicum & Seed & & [478] \\
\hline \multirow[t]{2}{*}{498} & Stigmasta-5,24(28)-dien-3-ol & S. elaeagnifolium & Seed & & [134] \\
\hline & & S. torvum & Leaf & & [318] \\
\hline 499 & Avenasterol & S. elaeagnifolium & Seed & & [134] \\
\hline 500 & 5,24-Stigmastadienol & S. elaeagnifolium & Seed & & [134] \\
\hline \multirow[t]{2}{*}{501} & $\gamma$-Tocopherol & S. lycopersicum & Seed & & [478] \\
\hline & & S. villosum & Leaf & & [479] \\
\hline 502 & Ergosterol & S. lycopersicum & Seed & & [478] \\
\hline 503 & Lanosterol & S. lycopersicum & Seed & & [478] \\
\hline \multirow[t]{2}{*}{504} & Peroxyergosterol & S. lyratum & Stem & & [486] \\
\hline & & S. violaceum & Aerial & & [391] \\
\hline \multirow[t]{2}{*}{505} & 9,11-Dehydroergosterol peroxide & S. lyratum & Stem & & [486] \\
\hline & & S. violaceum & Aerial & & [391] \\
\hline 506 & Nigralanostenone & S. nigrum & Leaf & & {$[528]$} \\
\hline 507 & Tumacone A & S. nudum & Leaf & & {$[242,247]$} \\
\hline 508 & Tumacone B & S. nudum & Leaf & & {$[242,247]$} \\
\hline 509 & Tumacoside A & S. nudum & Leaf & Antiplasmodial & {$[242,247]$} \\
\hline 510 & Tumacoside B & S. nudum & Leaf & Antiplasmodial & {$[242,247]$} \\
\hline 511 & SN-1 & S. nudum & Aerial & Antiplasmodial & [245] \\
\hline
\end{tabular}


Table 2 (continued)

\begin{tabular}{|c|c|c|c|c|c|}
\hline No. & Compounds & Plant sources & Parts & Biological properties & References \\
\hline 512 & $\mathrm{SN}-2$ & S. nudum & Aerial & Antiplasmodial & [245] \\
\hline 513 & $\mathrm{SN}-3$ & S. nudum & Aerial & Antiplasmodial & [245] \\
\hline 514 & $\mathrm{SN}-4$ & S. nudum & Aerial & Antiplasmodial & {$[245]$} \\
\hline 515 & $\mathrm{SN}-5$ & S. nudum & Aerial & Antiplasmodial & [245] \\
\hline 516 & $9 \alpha, 11 \alpha$-epidioxyergosta-6,22-dien-3 $\beta$-ol & S. septemlobum & Aerial & & [523] \\
\hline \multirow[t]{2}{*}{517} & \multirow[t]{2}{*}{ Carpesterol } & S. capsicoides & Seed & \multirow[t]{2}{*}{ Anticancer, antifungal } & [86] \\
\hline & & S. sisymbriifolium & Fruit & & [519] \\
\hline 518 & Carpesterol methyl ether & S. xanthocarpum & Fruit & Antifungal & [401] \\
\hline 519 & Carpesterol ethyl ether & S. xanthocarpum & Fruit & Antifungal & [401] \\
\hline 520 & $\begin{array}{l}\text { Stigmast-7-en-6-one, } 3 \text { - } \beta \text {-D- } \\
\text { glucosyloxy)22-hydroxy-4-methyl- } \\
(3 \beta, 4 \alpha, 5 \alpha, 22 \mathrm{R})\end{array}$ & S. xanthocarpum & Fruit & Antifungal & [401] \\
\hline 521 & $\begin{array}{l}\text { Stigmast-7-en-6-one, } 3-\beta \text {-D- } \\
\text { glucosyloxy)22-methoxy-4-methyl- } \\
(3 \beta, 4 \alpha, 5 \alpha, 22 \mathrm{R})\end{array}$ & S. xanthocarpum & Fruit & Antifungal & [401] \\
\hline 522 & Toptriol & S. glaucophyllum & Leaf & & [529] \\
\hline 523 & Cholecalciferol & S. glaucophyllum & Leaf & & {$[530]$} \\
\hline 524 & $\begin{array}{l}\beta \text {-D-Glucoside, }(1 \alpha, 3 \beta, 5 Z, 7 E)-3,25- \\
\text { dihydroxy-9,10-secocholesta -5,7,10(19) } \\
\text {-trien -1-yl }\end{array}$ & S. glaucophyllum & Leaf & & {$[530]$} \\
\hline 525 & Dehydrocholesterol & & & & \\
\hline 526 & $\begin{array}{l}\text { 3,4-Dihydro-3,5,8-trimethyl-3-(4,8,12- } \\
\text { trimethyltridecyl)-2H-1-benzopyran-7-yl } \\
\text { acetate }\end{array}$ & S. villosum & Leaf & & [479] \\
\hline 527 & Tumaquenone & S. nudum & Aerial & & [247] \\
\hline 528 & Abutiloside A & S. abutiloides & Root & & {$[5,7-9]$} \\
\hline 529 & Abutiloside B & S. abutiloides & Root & & [5] \\
\hline 530 & Abutiloside $\mathrm{H}$ & S. abutiloides & Root & & [5] \\
\hline 531 & Abutiloside I & S. abutiloides & Root & & [5] \\
\hline 532 & Abutiloside J & S. abutiloides & Root & & [5] \\
\hline 533 & Abutiloside K & S. abutiloides & Root & & [5] \\
\hline 534 & Abutiloside C & S. abutiloides & Root & & {$[7,8]$} \\
\hline 535 & Abutiloside D & S. abutiloides & Root & & [6] \\
\hline 536 & Abutiloside E & S. abutiloides & Root & & [6] \\
\hline 537 & Abutiloside F & S. abutiloides & Root & & [6] \\
\hline 538 & Abutiloside G & S. abutiloides & Root & & [6] \\
\hline 539 & Aethioside A & S. aethiopicum & Stem & & [28] \\
\hline 540 & Aethioside B & S. aethiopicum & Stem & & [28] \\
\hline \multirow[t]{2}{*}{541} & Aethioside C & S. aethiopicum & Stem & & [28] \\
\hline & Phenolic compounds & & & & \\
\hline \multirow[t]{2}{*}{542} & 4-Caffeoylquinic acid & S. melongena & Stem,Leaf & & {$[205,531]$} \\
\hline & & S. lyratum & Whole & & [517] \\
\hline \multirow[t]{2}{*}{543} & 5-Caffeoylquinic acid & S. melongena & Stem & & [205] \\
\hline & & S. sessiliflorum & Fruit & & {$[525]$} \\
\hline 544 & $\begin{array}{l}(1 R, 3 R, 4 S, 5 R)-3 \text {-(Acetyloxy)-5-[[(2E)-3- } \\
\text { (3,4-dihydroxyphenyl)-1-oxo-2-propen- } \\
\text { 1-yl]oxy] -1,4-dihydroxycyclohexanecar- } \\
\text { boxylic acid }\end{array}$ & S. melongena & Stem & & [205] \\
\hline 545 & $\begin{array}{l}(1 S, 3 R, 4 R, 5 R)-3 \text {-(Acetyloxy)-4-[[(2E)-3- } \\
\text { (3,4-dihydroxyphenyl)-1-oxo-2-propen- } \\
\text { 1-yl]oxy] -1,5-dihydroxycyclohexanecar- } \\
\text { boxylic acid }\end{array}$ & S. melongena & Stem & & [205] \\
\hline
\end{tabular}


Table 2 (continued)

\begin{tabular}{|c|c|c|c|c|c|}
\hline No. & Compounds & Plant sources & Parts & Biological properties & References \\
\hline \multirow[t]{7}{*}{546} & \multirow[t]{7}{*}{ Chlorogenic acid } & S. anguvi & Fruit & \multirow[t]{12}{*}{ Anticancer } & [31] \\
\hline & & S. guaraniticum & Leaf & & [146] \\
\hline & & S. incanum & Aerial & & [494] \\
\hline & & S. lycocarpum & Fruit & & [532] \\
\hline & & S. lyratum & Whole & & [517] \\
\hline & & S. melongena & Stem,Leaf & & {$[205,531]$} \\
\hline & & S. surattense & Whole & & [99] \\
\hline 547 & Neochlorogenic acid & S. lyratum & Whole & & {$[517]$} \\
\hline \multirow[t]{2}{*}{548} & \multirow[t]{2}{*}{ Rosmarinic acid } & S. betaceum & Fruit & & [78] \\
\hline & & S. guaraniticum & Leaf & & [146] \\
\hline 549 & 3,5-Dicaffeoylquinic acid & S. melongena & Stem & & [91] \\
\hline 550 & (Z)-Neochlorogenic acid & S. melongena & Stem & & [91] \\
\hline \multirow[t]{4}{*}{551} & \multirow[t]{4}{*}{ Gallic acid } & S. anguvi & Fruit & \multirow[t]{13}{*}{ Anticancer } & [31] \\
\hline & & S. сегпиит & Leaf & & [112] \\
\hline & & S. spirale & Aerial & & [299] \\
\hline & & S. surattense & Whole & & [99] \\
\hline \multirow[t]{2}{*}{552} & \multirow[t]{2}{*}{ 4-hydroxybenzoic acid } & S. crinitum & Fruit & & [122] \\
\hline & & S. americanum & Aerial & & [49] \\
\hline \multirow[t]{3}{*}{553} & \multirow[t]{3}{*}{ Protocatechuic acid } & S. lyratum & Whole & & [514] \\
\hline & & S. spirale & Leaf & & [297] \\
\hline & & S. nigrum & Whole & & {$[520]$} \\
\hline \multirow[t]{4}{*}{554} & \multirow[t]{4}{*}{ Vanillic acid } & S. lyratum & Whole & & [514] \\
\hline & & S. sessiliflorum & Fruit & & [510] \\
\hline & & S. nigrum & Whole & & {$[520]$} \\
\hline & & S. vestissimum & Fruit & & [491] \\
\hline \multirow[t]{9}{*}{555} & \multirow[t]{9}{*}{ Caffeic acid } & S. anguvi & Fruit & \multirow[t]{23}{*}{ Anticancer } & [31] \\
\hline & & S. guaraniticum & Leaf & & [146] \\
\hline & & S. incanum & Aerial & & {$[506]$} \\
\hline & & S. lycocarpum & Fruit & & [532] \\
\hline & & S. lyratum & Whole & & [194] \\
\hline & & S. melongena & Stem & & [205] \\
\hline & & S. muricatum & Whole & & [215] \\
\hline & & S. surattense & Whole & & {$[99,518]$} \\
\hline & & S. xanthocarpum & Root & & [427] \\
\hline 556 & $P$-Coumaric acid & S. americanum & Aerial & & [49] \\
\hline 557 & Isoferulic acid & S. сегпиит & Leaf & & {$[109,112]$} \\
\hline 558 & 2,4,6-Trimethoxyphenol & S. torvum & Stem & & [533] \\
\hline 559 & Propionylsyringol & S. torvum & Stem & & {$[533]$} \\
\hline 560 & Resveratrol & S. americanum & Fruit & & [45] \\
\hline 561 & cis-p-Coumaric acid ethyl ester & S. crinitum & Fruit & & {$[122]$} \\
\hline 562 & $c i s-p$-Coumaric acid & S. crinitum & Fruit & & [122] \\
\hline 563 & trans- $p$-Coumaric acid ethyl ester & S. crinitum & Fruit & & [122] \\
\hline \multirow[t]{2}{*}{564} & \multirow[t]{2}{*}{ trans- $p$-Coumaric acid } & S. crinitum & Fruit & & [122] \\
\hline & & S. incanum & Aerial & & {$[506]$} \\
\hline 565 & $\begin{array}{l}\text { Erythro-1,2-bis-(4-hydroxy- } \\
\text { 3-methoxyphenyl)-1,3-propanediol }\end{array}$ & S. lyratum & Whole & & {$[517]$} \\
\hline 566 & $\begin{array}{l}\text { Threo-1,2-bis-(4-hydroxy- } \\
\text { 3-methoxyphenyl)-1,3-propanediol }\end{array}$ & S. lyratum & Whole & & [517] \\
\hline 567 & Evofolin B & S. surattense & Whole & & {$[518]$} \\
\hline 568 & Ethyl caffeate & S. nienkui & Whole & & [509] \\
\hline
\end{tabular}


Table 2 (continued)

\begin{tabular}{|c|c|c|c|c|c|}
\hline No. & Compounds & Plant sources & Parts & Biological properties & References \\
\hline \multirow[t]{2}{*}{569} & \multirow[t]{2}{*}{ Methyl salicylate } & S. nienkui & Whole & & [509] \\
\hline & & S. aculeastrum & Leaf & & [11] \\
\hline 570 & p-Hydroxybenzoic acid & S. nigrum & Whole & & {$[520]$} \\
\hline 571 & Vanillin & S. nienkui & Whole & & [509] \\
\hline 572 & Protocatechuic aldehyde & S. nienkui & Whole & & [509] \\
\hline 573 & 3,5-Diethoxyphenol & S. nigrum & Leaf & & {$[528]$} \\
\hline 574 & Quinic acid & S. sessiliflorum & Fruit & & {$[525]$} \\
\hline 575 & Phenol & S. sessiliflorum & Fruit & & [525] \\
\hline 576 & Salicylic acid & S. torvum & Aerial & & [524] \\
\hline 577 & Violaxanthin & S. sessiliflorum & Fruit & & [525] \\
\hline 578 & Lutein & S. sessiliflorum & Fruit & & {$[525]$} \\
\hline 579 & $\alpha$-Carotene & S. sessiliflorum & Fruit & & {$[525]$} \\
\hline 580 & Kryptoxanthin & S. sessiliflorum & Fruit & & {$[525]$} \\
\hline 581 & Luteoxanthin & S. sessiliflorum & Fruit & & {$[525]$} \\
\hline 582 & 15 -cis- $\beta$-Carotene & S. sessiliflorum & Fruit & & {$[525]$} \\
\hline 583 & Foliaxanthin & S. sessiliflorum & Fruit & & {$[525]$} \\
\hline 584 & Physoxanthin & S. sessiliflorum & Fruit & & {$[525]$} \\
\hline 585 & Coniferol & S. surattense & Whole & & {$[518]$} \\
\hline 586 & $\begin{array}{l}\text { 1,2-Bis(4-hydroxy-3-methoxyphenyl)- } \\
\text { 1,3-propanediol }\end{array}$ & S. surattense & Whole & & [518] \\
\hline 587 & $\begin{array}{l}\text { Threo-1-(4-Hydroxy-3-methoxyphenyl)-2- } \\
\text { [4-[(E)-3-hydroxy-1-propenyl]-2-meth- } \\
\text { oxy phenoxy]-1,3-propanediol }\end{array}$ & S. surattense & Whole & & {$[518]$} \\
\hline 588 & Tyrosol C & S. validinervium & Aerial & & {$[534]$} \\
\hline 589 & (E)-Coniferaldehyde & S. melongena & Root & & [209] \\
\hline 590 & trans-Cinnamic acid & S. spirale & Leaf & Antibacterial & [297] \\
\hline 591 & Methyl caffeate & S. torvum & Fruit & Antibacterial,antidiabetic & $\begin{array}{l}{[315,320,} \\
335-337]\end{array}$ \\
\hline 592 & $\begin{array}{l}\text { (E)-2,3-dihydroxycyclopentyl-3-(3',4'- } \\
\text { dihydroxyphenyl)acrylate }\end{array}$ & S. torvum & Fruit & Antihypertensive & {$[521]$} \\
\hline \multirow[t]{2}{*}{593} & Eugenol & S. torvum & Stem & & {$[533]$} \\
\hline & Coumarins and coumestans & & & & \\
\hline \multirow[t]{3}{*}{594} & \multirow[t]{3}{*}{ Scopolin } & S. cathayanum & Stem & Anticancer & {$[104,105]$} \\
\hline & & S. lyratum & Whole & & [194] \\
\hline & & S. septemlobum & Aerial & & [523] \\
\hline \multirow[t]{3}{*}{595} & \multirow[t]{3}{*}{ Scopoletin } & S. glabratum & Whole & & {$[140]$} \\
\hline & & S. indicum & Seed & & {$[535]$} \\
\hline & & S. ligustrinum & Aerial & & [179] \\
\hline \multirow[t]{3}{*}{596} & \multirow[t]{3}{*}{ Coumarin } & S. incanum & Leaf & & [494] \\
\hline & & S. surattense & Whole & & [99] \\
\hline & & S. vestissimum & Fruit & & [491] \\
\hline 597 & Fraxetin & S. indicum & Seed & & [536] \\
\hline 598 & Isofraxidin & S. indicum & Seed & & [536] \\
\hline 599 & Umbelliferone & S. lycopersicum & Aerial & & [438] \\
\hline 600 & $\begin{array}{l}\text { 7-hydroxy-6,8-dimethoxy-3-(4'-hydroxy- } \\
\text { 3'-methoxyphenyl)-coumarin }\end{array}$ & S. indicum & Seed & & {$[536]$} \\
\hline 601 & Cleosandrin & S. indicum & Seed & & {$[535]$} \\
\hline 602 & 4,4'-Biisofraxidin & S. indicum & Seed & & [535] \\
\hline 603 & Arteminorin A & S. indicum & Seed & & [535] \\
\hline 604 & Indicumin E & S. indicum & Seed & & {$[536]$} \\
\hline 605 & Bergaptin & S. lycopersicum & Aerial & & [438] \\
\hline
\end{tabular}


Table 2 (continued)

\begin{tabular}{|c|c|c|c|c|c|}
\hline No. & Compounds & Plant sources & Parts & Biological properties & References \\
\hline \multirow[t]{2}{*}{606} & \multirow[t]{2}{*}{ Aesculetin } & S. lycopersicum & Aerial & & [438] \\
\hline & & S. validinervium & Aerial & & {$[534,537]$} \\
\hline 607 & 6, 7-Dimethoxycoumarin & S. melongena & Root & & [209] \\
\hline 608 & Escopoletin & S. nigrum & Whole & & {$[520]$} \\
\hline 609 & Isoscopoletin & S. validinervium & Aerial & & {$[534,537]$} \\
\hline 610 & $\begin{array}{l}\text { 1'-O-7-esculetin-4'-O-1"-ethylenglycol- } \beta \text { - } \\
\text { D-glucose }\end{array}$ & S. validinervium & Aerial & & [534] \\
\hline 611 & Coumestrol & S. lyratum & Whole & Anti-inflammatory & [88] \\
\hline 612 & $\begin{array}{l}\text { 9-hydroxy-2',2'-dimethyl[5',6':2,3]- } \\
\text { coumestan }\end{array}$ & S. lyratum & Whole & Anti-inflammatory & [88] \\
\hline \multirow[t]{2}{*}{613} & Solalyratin A & S. lyratum & Whole & Anti-inflammatory & [88] \\
\hline & \multicolumn{5}{|l|}{ Coumarinolignoids } \\
\hline 614 & Indicumine A & S. indicum & Seed & Anti-HBV & {$[535]$} \\
\hline 615 & Indicumine B & S. indicum & Seed & Anti-HBV & {$[535]$} \\
\hline 616 & Indicumine $\mathrm{C}$ & S. indicum & Seed & & {$[535]$} \\
\hline \multirow[t]{2}{*}{617} & Indicumine D & S. indicum & Seed & & {$[535]$} \\
\hline & \multicolumn{5}{|l|}{ Fatty acids and esters } \\
\hline \multirow[t]{3}{*}{618} & \multirow[t]{3}{*}{ Hexadecanoic acid } & S. aculeastrum & Leaf & & [11] \\
\hline & & S. vestissimum & Fruit & & [490] \\
\hline & & S. villosum & Leaf & & {$[434,479]$} \\
\hline \multirow[t]{2}{*}{619} & \multirow[t]{2}{*}{ Octadecanoic acid, } & S. aculeastrum & Leaf & & {$[11]$} \\
\hline & & S. erianthum & Leaf & & [137] \\
\hline \multirow[t]{2}{*}{620} & \multirow[t]{2}{*}{ Linoleic acid } & S. aculeastrum & Leaf & & [11] \\
\hline & & S. glabratum & Whole & & {$[140]$} \\
\hline 621 & Lignoceric acid & S. cathayanum & Stem & & [477] \\
\hline 622 & Corchorifatty acid B & S. americanum & Aerial & & [49] \\
\hline \multirow[t]{2}{*}{623} & \multirow[t]{2}{*}{ Linolenic acid } & S. erianthum & Leaf & & {$[137]$} \\
\hline & & S. glabratum & Whole & & [140] \\
\hline 624 & $9(Z), 11(E)$-Octadecadienoic acid & S. erianthum & Leaf & & [137] \\
\hline 625 & $\begin{array}{l}13 S \text {-Hydroxy- } 9(Z), 11(E) \text {-octadecadienoic } \\
\text { acid }\end{array}$ & S. erianthum & Leaf & & [137] \\
\hline 626 & $\begin{array}{l}9 S \text {-Hydroxy-10(E),12(Z),15(Z)-octadec- } \\
\text { atrienoic acid }\end{array}$ & S. erianthum & Leaf & & [137] \\
\hline 627 & Decosahexaenoic acid & S. glabratum & Whole & & {$[140]$} \\
\hline 628 & Decosapentaenoic acid & S. glabratum & Whole & & [140] \\
\hline 629 & Oleic acid & S. glabratum & Whole & & {$[140]$} \\
\hline 630 & Eicosapentaenoic acid & S. glabratum & Whole & & [140] \\
\hline 631 & Lauric acid & S. glabratum & Whole & & {$[140]$} \\
\hline 632 & Palmitoleic acid & S. glabratum & Whole & & {$[140]$} \\
\hline \multirow[t]{2}{*}{633} & \multirow[t]{2}{*}{ Arachidonic acid } & S. glabratum & Whole & & [140] \\
\hline & & S. trilobatum & Whole & & [356] \\
\hline 634 & Myristic acid & S. glabratum & Whole & & [140] \\
\hline 635 & Gamma-linolenic acid & S. glabratum & Whole & & {$[140]$} \\
\hline 636 & 9-Oxо-(10E, 12Z)-octadecadienoic acid & S. melongena & Calyx & & [91] \\
\hline 637 & $\begin{array}{l}(10 Z, 12 E)-9-O x o-10,12 \text {-octadecadienoic } \\
\text { acid }\end{array}$ & S. melongena & Calyx & & [91] \\
\hline 638 & Eicosanoic acid & S. torvum & Root & & {$[526]$} \\
\hline 639 & Octacosanoic acid & S. torvum & Root & & [526] \\
\hline \multirow[t]{2}{*}{640} & $\begin{array}{l}\text { 4-(3,5-Di-Tert-Butyl-4-Hydroxy Phenyl) } \\
\text { butyl Acrylate }\end{array}$ & S. villosum & Leaf & & [479] \\
\hline & Others & & & & \\
\hline
\end{tabular}


Table 2 (continued)

\begin{tabular}{|c|c|c|c|c|c|}
\hline No. & Compounds & Plant sources & Parts & Biological properties & References \\
\hline 641 & Puerariafuran & S. lyratum & Whole & Anti-inflammatory & [88] \\
\hline 642 & 1,2-Benzenedicarboxylic acid & S. aculeastrum & Leaf & & [11] \\
\hline 643 & 1, 4-Dimethyl-benzene & S. aculeastrum & Leaf & & [11] \\
\hline 644 & $n$-Nonane & S. aculeastrum & Leaf & & [11] \\
\hline 645 & $n$-Octanol & S. aculeastrum & Leaf & & {$[11]$} \\
\hline 646 & Methyl hexadecanoate & S. aculeastrum & Leaf & & [11] \\
\hline 647 & Dodecane & S. aculeastrum & Leaf & & [11] \\
\hline 648 & Undecanal & S. aculeastrum & Leaf & & [11] \\
\hline 649 & Nonanal & S. aculeastrum & Leaf & & {$[11]$} \\
\hline \multirow[t]{2}{*}{650} & Eicosane & S. aculeastrum & Leaf & & {$[11]$} \\
\hline & & S. betaceum & Fruit & & {$[77]$} \\
\hline 651 & Methyl-9,12-octadecadienoate & S. aculeastrum & Leaf & & {$[11]$} \\
\hline 652 & Hexadecane & S. aculeastrum & Leaf & & {$[11]$} \\
\hline 653 & 9,17-Octadecadienal & S. aculeastrum & Leaf & & [11] \\
\hline 654 & Hexanal & S. betaceum & Fruit & & {$[78]$} \\
\hline 655 & Ethyl butanoate & S. betaceum & Fruit & & {$[78]$} \\
\hline 656 & 4-Hydroxy-4-methyl-2-pentanone & S. betaceum & Fruit & & [78] \\
\hline 657 & 2,3-Butanediol & S. betaceum & Fruit & & [78] \\
\hline 658 & cis-3-Hexen-1-ol & S. betaceum & Fruit & & [78] \\
\hline 659 & 3(Z)-Hexenal & S. betaceum & Fruit & & [78] \\
\hline 660 & Ethyl- $\alpha$-D-arabinofuranoside & S. lyratum & Whole & & [514] \\
\hline 661 & Solalyratin B & S. lyratum & Whole & Anti-inflammatory & [88] \\
\hline 662 & $\begin{array}{l}\text { 1- }\{1-[2-(2 \text { hydroxypropoxy) propoxy] } \\
\text { propan-2-yloxy }\} \text { propan-2-ol }\end{array}$ & S. schimperianum & Aerial & & [277] \\
\hline 663 & 5-Hydroxymethyl furfural & S. torvum & Stem & & {$[533]$} \\
\hline 664 & Solanesol & S. tuberosum & Leaf & & [538] \\
\hline 665 & 3-Hydroxymethyl-7-methoxywutaifuranol & S. cathayanum & Whole & & [102] \\
\hline 666 & $\begin{array}{l}\text { Phenylmethyl } 2-O-\beta \text {-D-xylosyl- } \beta \text {-D- } \\
\text { glucoside }\end{array}$ & S. incanum & Aerial & & [506] \\
\hline 667 & Zizybeoside I & S. lycopersicum & Fruit & & {$[511]$} \\
\hline 668 & $\begin{array}{l}\text { Methyl salicylate } 2-O-\beta \text {-D-glucosyl-(1-2)- } \\
{[\mathrm{O}-\beta \text {-D-xylosyl-(1-6)]- } O \text { - } \beta \text {-D-glucoside }}\end{array}$ & S. lycopersicum & Fruit & & [511] \\
\hline 669 & $\begin{array}{l}\text { Phenethyl alcohol 8-O- } \beta \text {-D-glucosyl-(1- } \\
\text { 2)-[O- } \alpha \text {-L-arabinosyl-(1-6)]-O- } \beta \text {-D- } \\
\text { glucoside }\end{array}$ & S. lycopersicum & Fruit & & [511] \\
\hline 670 & $\begin{array}{l}\text { Benzyl alcohol 7- } O-\beta \text {-D-glucosyl-(1-2)- } \\
{[O-\alpha \text {-L-arabinosyl-(1-6)]- } \beta \text {-D-glucoside }}\end{array}$ & S. lycopersicum & Fruit & & {$[511]$} \\
\hline
\end{tabular}

An avenacoside-type saponin (51) was isolated from aerial parts of $S$. surattense [305]. Two 23-keto-spirostanol glycosides, torvoside Q (18) and paniculonin B (126) were obtained from aerial parts of $S$. torvum $[323,331]$. Torvosides A (64), B (65), F (67) and G (112) displayed a positive reaction with Ehrlich reagent, suggesting these to be furostanol glycosides [449]. Abutilosides L (106), M (107) and $\mathrm{N}(\mathbf{1 0 8})$, a $22 S, 25 S$-epoxy-furost-5-ene type glycosides, and abutiloside O, being a 20,22-seco-type steroidal glycoside, were isolated from the fresh fruits of $S$. abutiloides [4].

Anguiviosides III (118) and XI (119) are hydroxylated at C-23 and C-26 on the spirostanol and furostanol skeletons, resp. Anguiviosides XV (120) and XVI (121) are based on a 16, 22-dicarbonyl aglycon, with $\mathbf{1 2 1}$ hydroxylated at C-23 and $\mathrm{C}-26$ followed by ring closure. The biogenetic pathway of 16,22-dicarbonyl compounds such as $\mathbf{1 2 0}$ and $\mathbf{1 2 1}$ might be considered via a $17 R$-hydroxy spirostanol such as pennogenin, 11 or via a $3 \beta, 16 \beta, 22,26$-tetrahydroxycholesterol glycoside such as anguivioside A (114) [43].

Solanum saponins were reported to have various bioactivies, e.g. cytotoxic [257], anticancer [316, 317, 392], hepatoprotective [242, 247], antihypertensive [289, 291], antimelanogenesis [211], antifungal [113, 114, 117], anti-inflammatory [331, 392, 448] anticonvulsant [305] and antiviral [257]. 


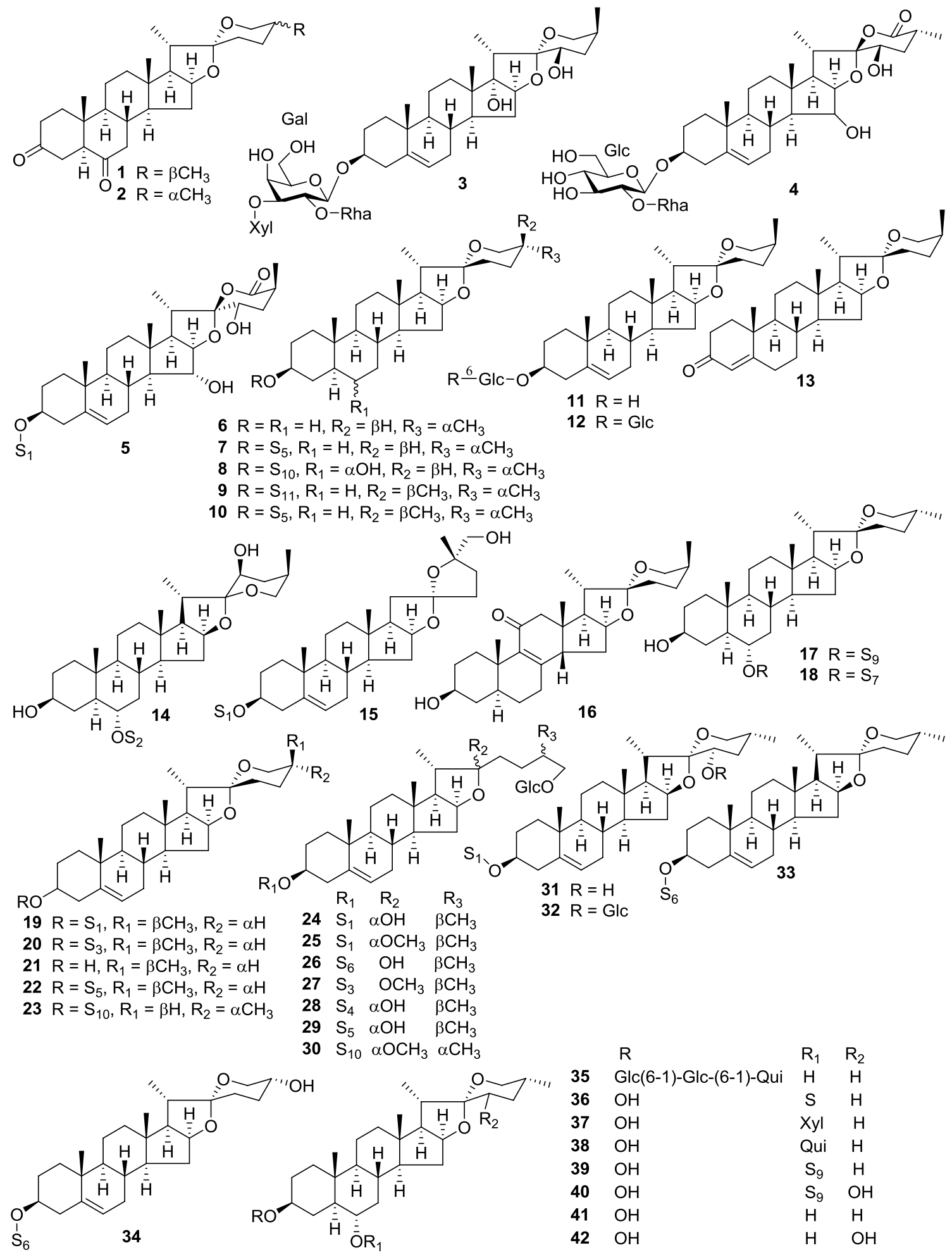

Fig. 1 Steroidal saponins 1-134 from Solanum

Nuatigenosido (15) from the roots of S. sisymbriifolium presented anti-hypertensive effect in experimental hypertensive rats [291]. Dioscin (19) showed antimelanogenesis effect on $\alpha$-melanocyte stimulating hormone ( $\alpha$-MSH) induced melanogenesis in B16 murine melanoma cells. It significantly downregulated the expression of tyrosinase, TRP-1, and TRP-2, which led to the reduction of $\alpha-\mathrm{MSH}-$ induced melanogenesis in B16 cells [211]. Degraded 

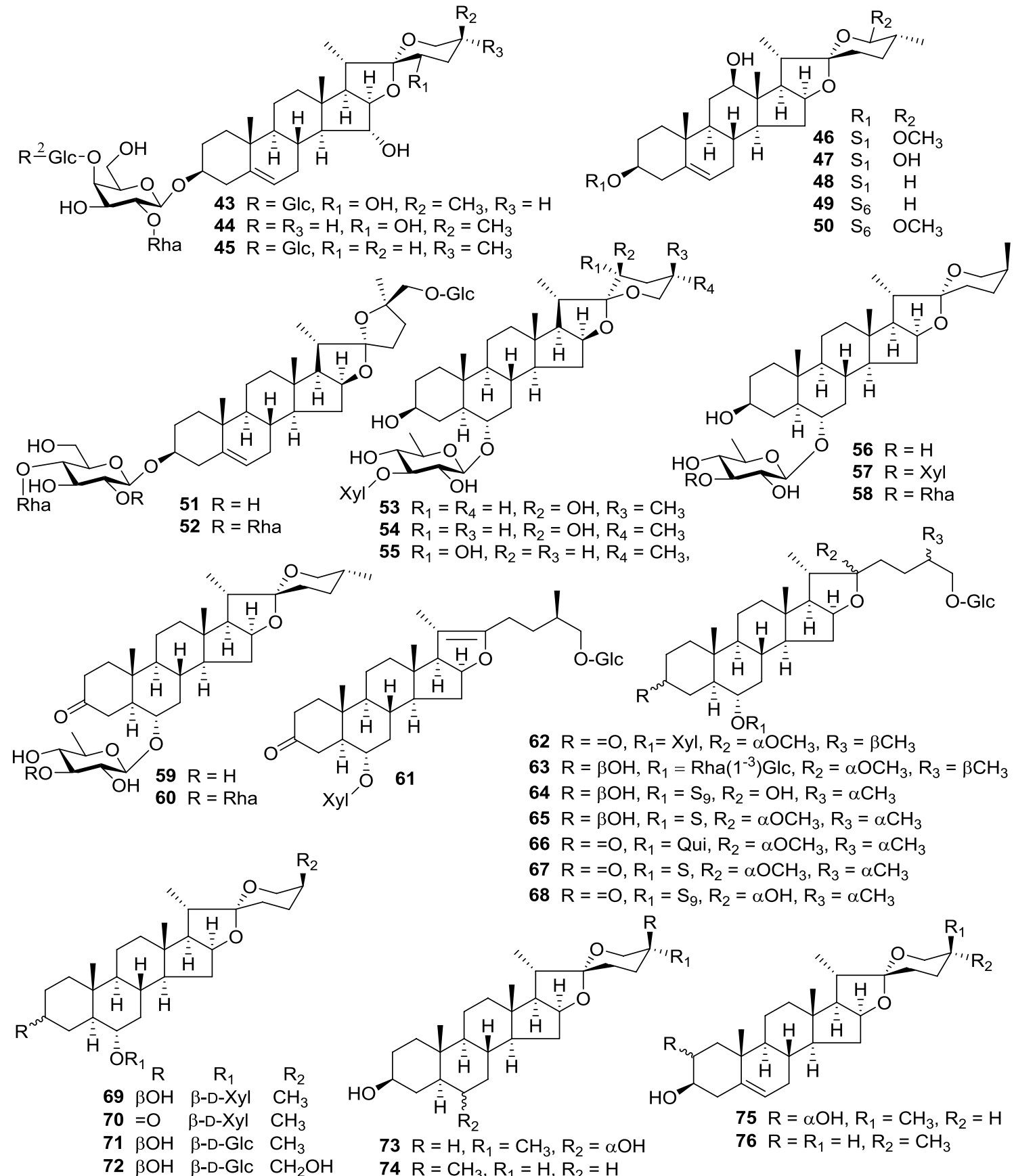

$$
\begin{aligned}
& =\mathrm{CH}_{3} \\
& =\mathrm{CH}_{3},
\end{aligned}
$$<smiles>CCC[C@H](C)CO[GeH3]</smiles>

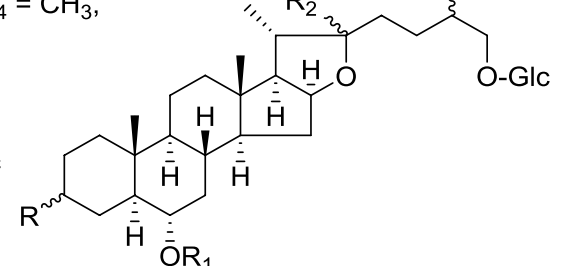
$62 \mathrm{R}==\mathrm{O}, \mathrm{R}_{1}=\mathrm{Xyl}, \mathrm{R}_{2}=\alpha \mathrm{OCH}_{3}, \mathrm{R}_{3}=\beta \mathrm{CH}_{3}$ $63 \mathrm{R}=\beta \mathrm{OH}, \mathrm{R}_{1}=\mathrm{Rha}\left(1^{-3}\right) \mathrm{Glc}, \mathrm{R}_{2}=\alpha \mathrm{OCH}_{3}, \mathrm{R}_{3}=\beta \mathrm{CH}_{3}$ $64 \mathrm{R}=\beta \mathrm{OH}, \mathrm{R}_{1}=\mathrm{S}_{9}, \mathrm{R}_{2}=\mathrm{OH}, \mathrm{R}_{3}=\alpha \mathrm{CH}_{3}$ $65 \mathrm{R}=\beta \mathrm{OH}, \mathrm{R}_{1}=\mathrm{S}, \mathrm{R}_{2}=\alpha \mathrm{OCH}_{3}, \mathrm{R}_{3}=\alpha \mathrm{CH}_{3}$ $66 \mathrm{R}==\mathrm{O}, \mathrm{R}_{1}=$ Qui, $\mathrm{R}_{2}=\alpha \mathrm{OCH}_{3}, \mathrm{R}_{3}=\alpha \mathrm{CH}_{3}$ $67 \mathrm{R}==\mathrm{O}, \mathrm{R}_{1}=\mathrm{S}, \mathrm{R}_{2}=\alpha \mathrm{OCH}_{3}, \mathrm{R}_{3}=\alpha \mathrm{CH}_{3}$ $68 \mathrm{R}==\mathrm{O}, \mathrm{R}_{1}=\mathrm{S}_{9}, \mathrm{R}_{2}=\alpha \mathrm{OH}, \mathrm{R}_{3}=\alpha \mathrm{CH}_{3}$

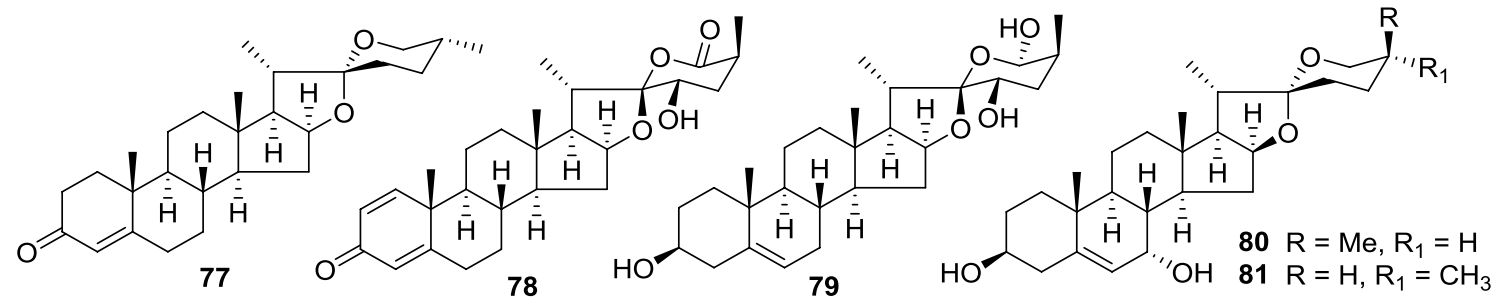

Fig. 1 (continued) 


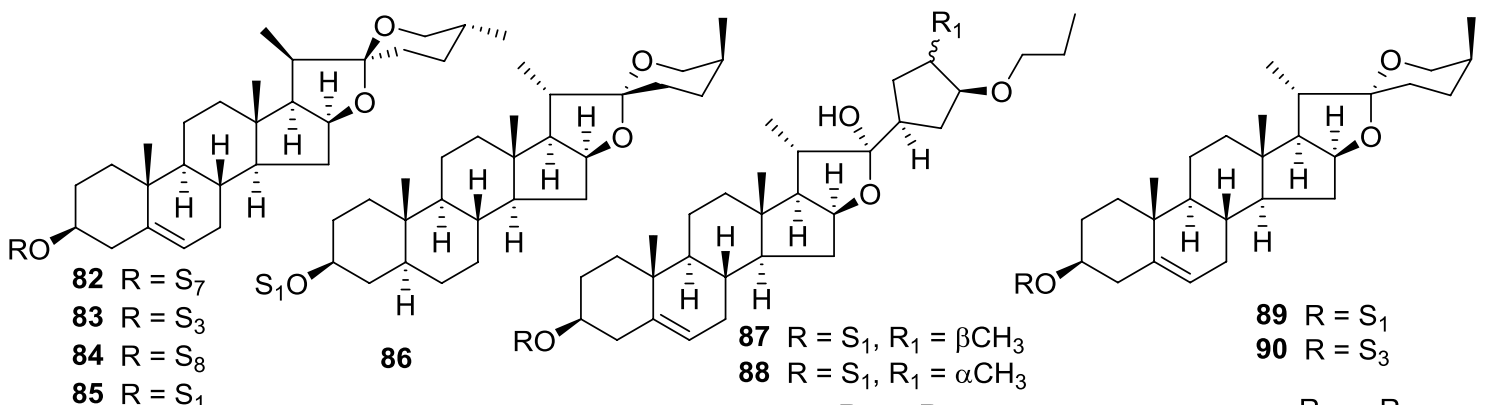

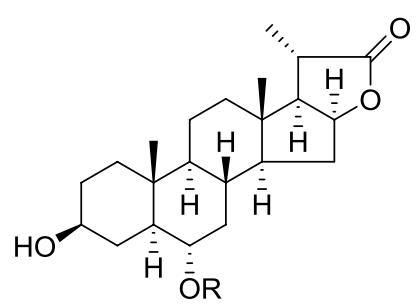

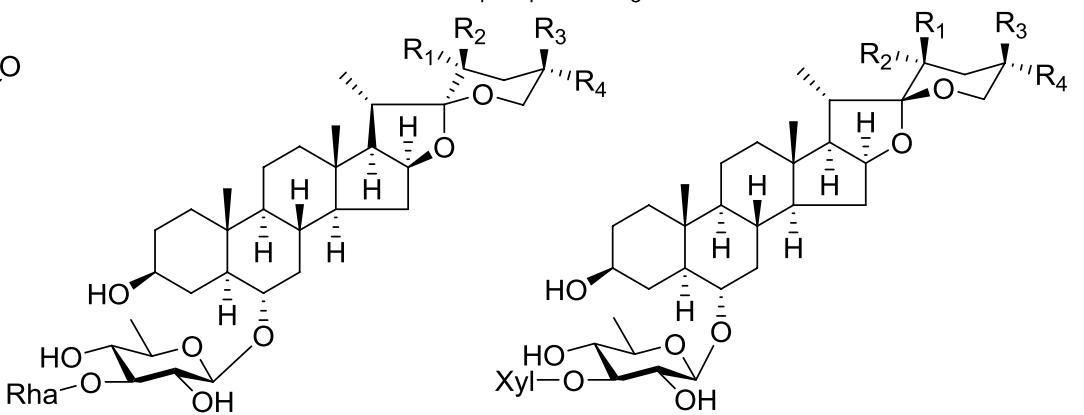
$95 \mathrm{R}_{1}=\mathrm{R}_{3}=\mathrm{H}, \mathrm{R}_{2}=\mathrm{OH}, \mathrm{R}_{4}=\mathrm{CH}_{3}$
$\begin{array}{rl}93 & R=\beta-D-Q u i \\ 94 R & =H\end{array}$
$96 \mathrm{R}_{1}=\mathrm{R}_{4}=\mathrm{H}, \mathrm{R}_{2}=\mathrm{OH}, \mathrm{R}_{3}=\mathrm{CH}_{3}$
$98 \mathrm{R}_{1}=\mathrm{OH}, \mathrm{R}_{2}=\mathrm{R}_{3}=\mathrm{H}, \mathrm{R}_{4}=\mathrm{CH}_{3}$
$97 \mathrm{R}_{1}=\mathrm{OH}, \mathrm{R}_{2}=\mathrm{R}_{3}=\mathrm{H}, \mathrm{R}_{4}=\mathrm{CH}_{3}$
$99 \mathrm{R}_{1}=\mathrm{OH}, \mathrm{R}_{2}=\mathrm{R}_{4}=\mathrm{H}, \mathrm{R}_{3}=\mathrm{CH}_{3}$
$100 R_{1}=R_{3}=H, R_{2}=O H, R_{4}=C_{3}$

$91 \mathrm{R}=\mathrm{S}$

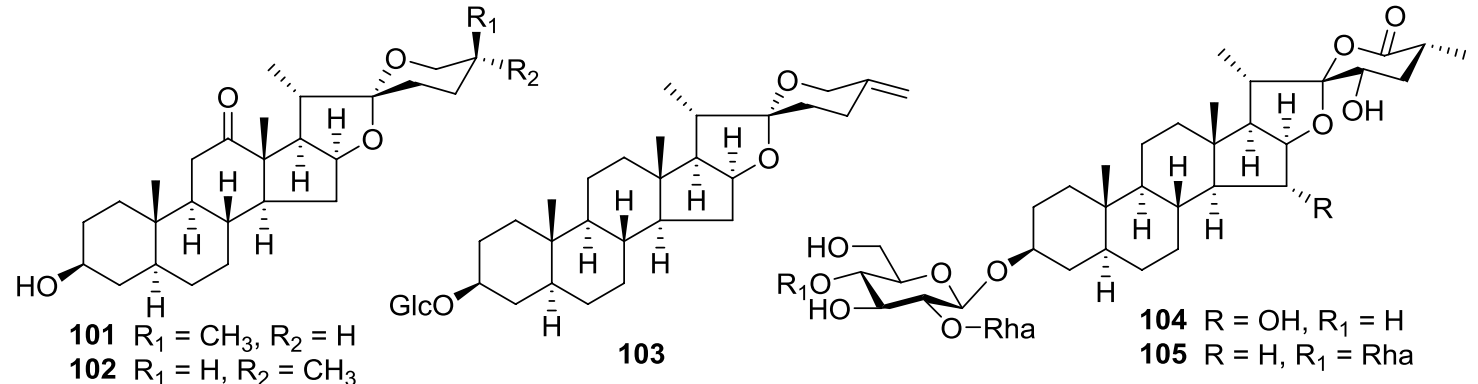

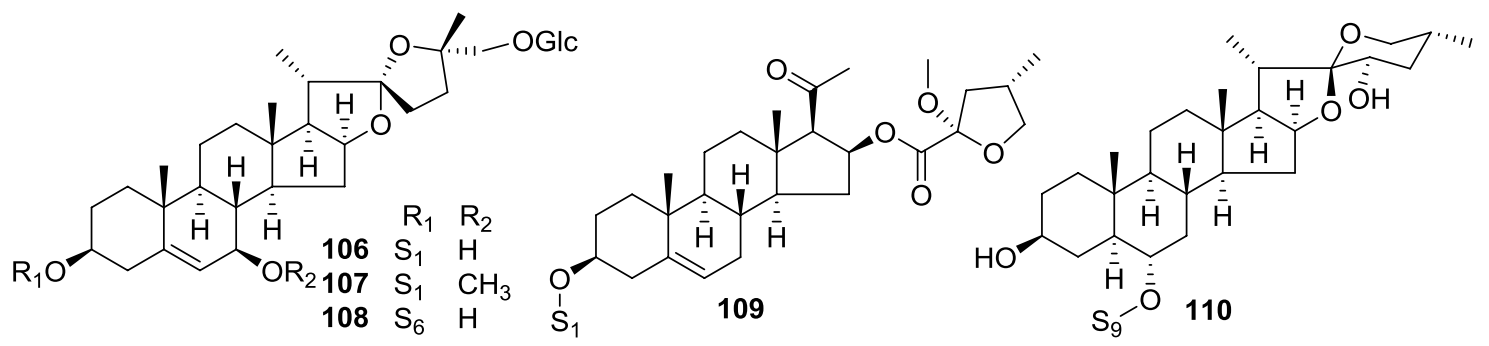

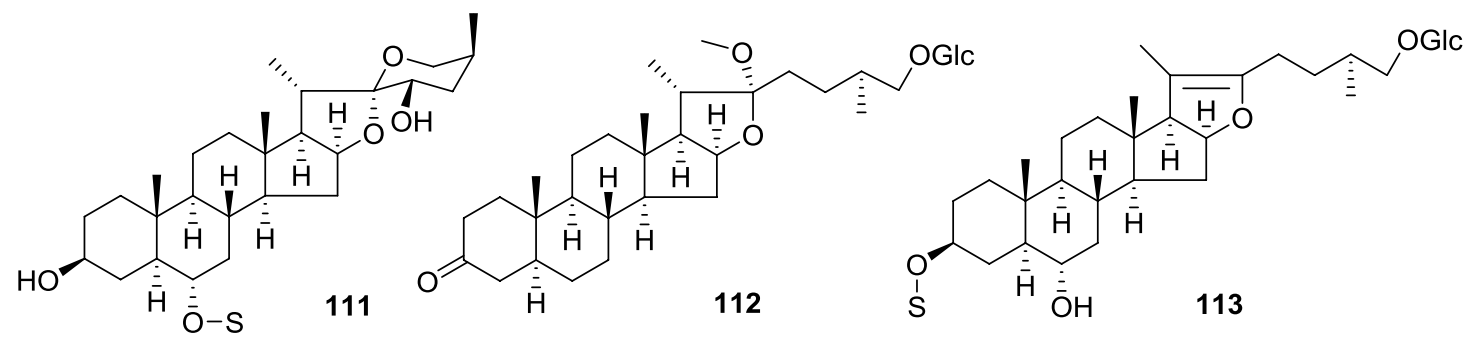

Fig. 1 (continued) 


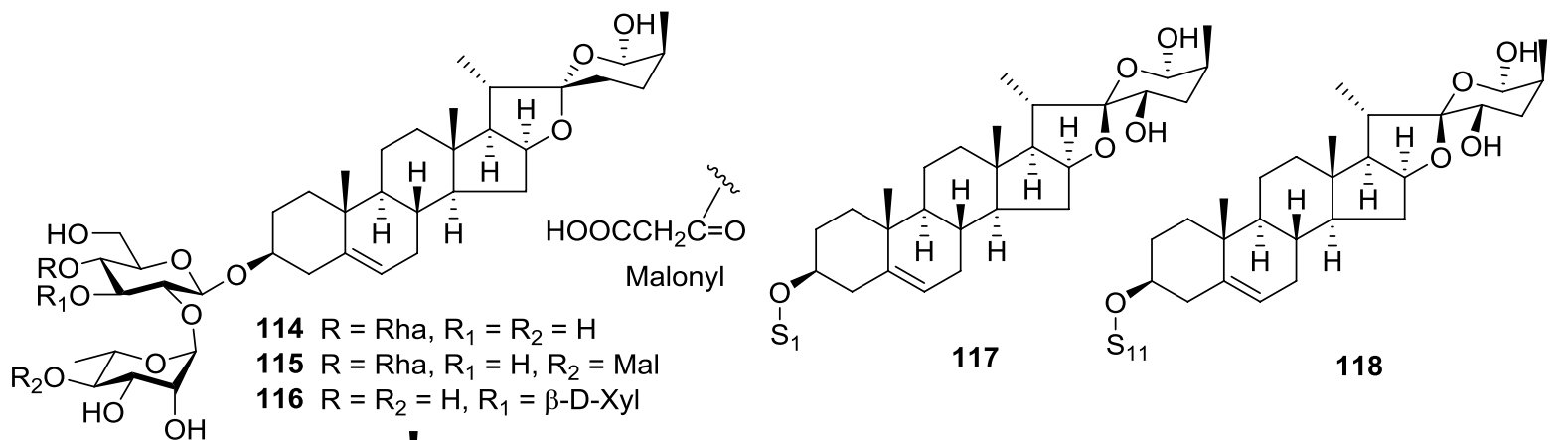

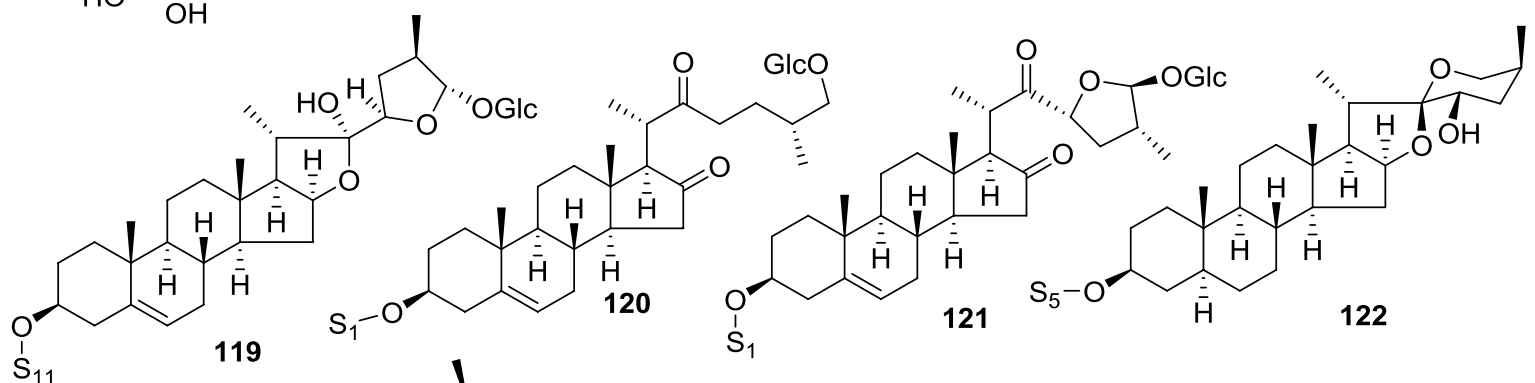

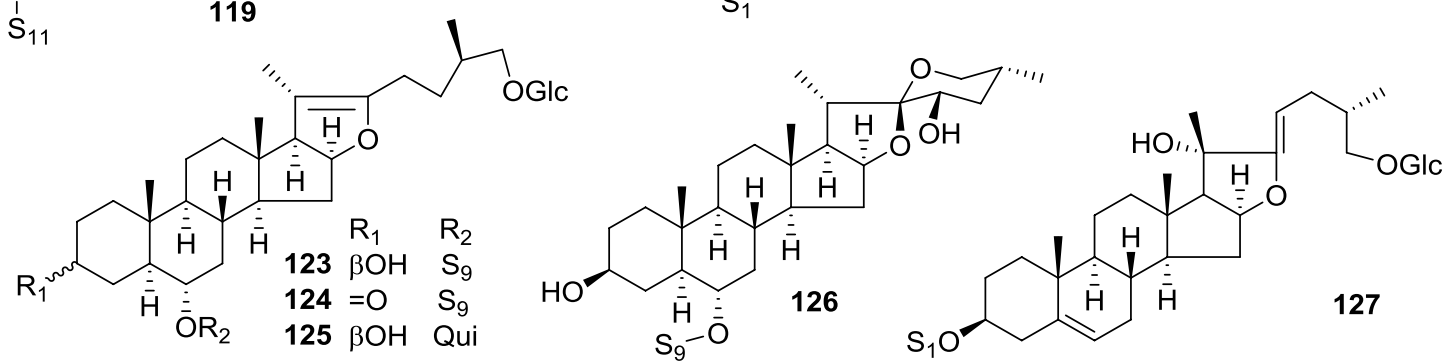

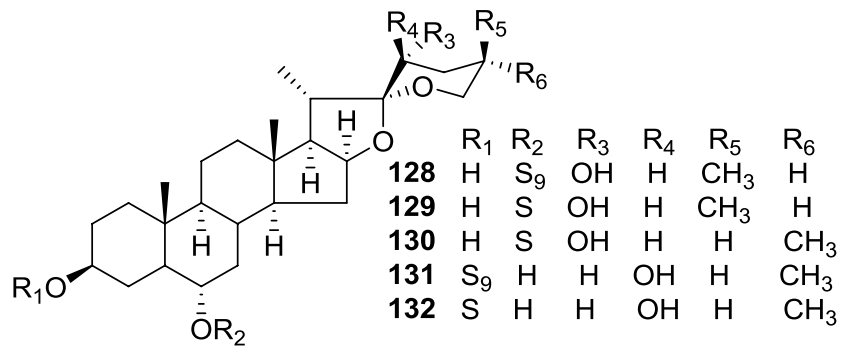

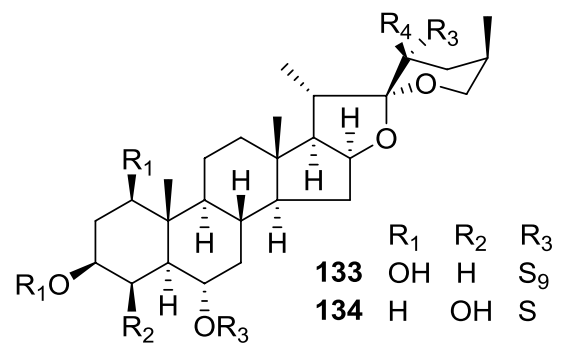

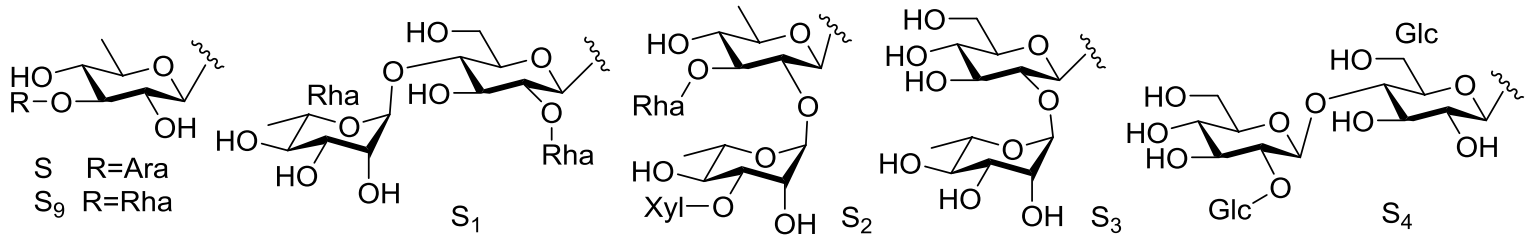

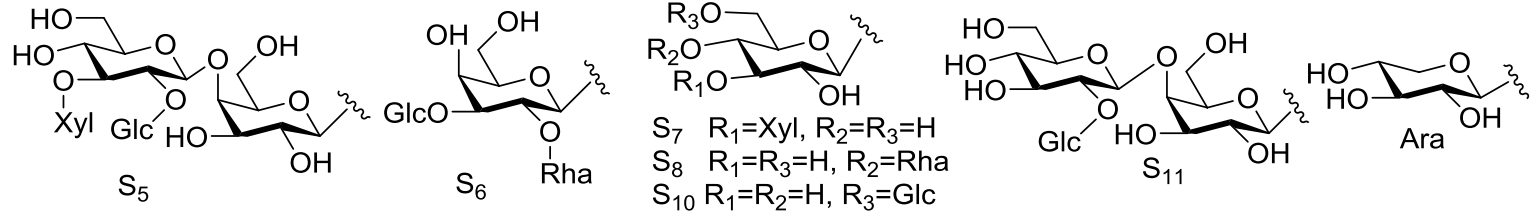

Fig. 1 (continued)

diosgenone (13) from $S$. nudum exhibited hepatoprotective effect on the liver of mice infected with Plasmodium berghei; necrosis of hepatocytes in mice infected with malaria decreased 47-65 [249].
Spirostanic saponins SC2-SC6 (36-40) from the leaves of S. chrysotrichum displayed activity against dermatophytes and yeasts. $\mathbf{3 6}$ was the most active in indicating fungicidal 
effect against Candida albicans and non-albicans strains $[113,114,117]$.

Indioside $\mathrm{H}(\mathbf{8 3})$, borassoside $\mathrm{E}(\mathbf{8 5})$, indioside I (86) and yamoscin (89) demonstrated cytotoxic activity against six human cancer cell lines (HepG2, Hep3B, A549, Ca922, MDA-MB-231, and MCF-7) $\left(\mathrm{IC}_{50}=1.83-8.04 \mu \mathrm{g} / \mathrm{mL}\right)$ [392]. Seperately, $\mathbf{8 5}$ and $\mathbf{8 6}$ presented inflammation inhibitory effects on SAG $\left(\mathrm{IC}_{50}=0.62 \pm 0.03\right.$ and $2.84 \pm 0.18 \mu \mathrm{g} /$ $\mathrm{mL}$, resp.). Compound 85 also inhibited elastase release with $\mathrm{IC}_{50}$ values of $111.05 \pm 7.37 \mu \mathrm{g} / \mathrm{mL}$ [392], while $\mathbf{8 9}$ showed anti-neutrophilic inflammatory activity against SAG with an $\mathrm{IC}_{50}$ value of $3.59 \mu \mathrm{M}$ [331].

Torvosides N (8) and M (23) revealed significant cytotoxicity against MGC-803, HepG2, A549 and MCF-7 as compared to the positive control, CDDP [316]. Torvosides J-L (95-97), isolated from the leaves of $S$. torvum, exhibited substantial anticonvulsant activity in zebrafish seizure assays [323], while 96 also showed considerable antifungal activity against Aspergillus flavus and Fusarium verticillioides with MIC ranging from 31.25 to $250 \mu \mathrm{g} / \mathrm{mL}$ [318]. Compounds 99 and 100 inhibited both inflammatory mediators SAG $\left(\mathrm{IC}_{50}=3.49\right.$ and $\left.2.87 \mu \mathrm{M}\right)$ and elastase release $\left(\mathrm{IC}_{50}=2.69\right.$ and $0.66 \mu \mathrm{M})$ [331], while 123-125 convinced cytotoxicities against melanoma A375 [317].

\subsection{Steroidal Alkaloids}

Sixty-three steroidal alkaloids (135-197), as other principal components in Solanum were reported from this genus (Fig. 2). Compounds 139-156 are derivatives of solasodine (145), one of the main glycoalkaloid constituents in Solanum spp., even as indicated by several numbers of species from which it has been isolated. Solamargine (139) is the major steroidal alkaloid constituent of Solanum plants and literature data showed that it has been revealed in 18 species.

Compounds such as 139, solasonine (142), $\beta 1$-solasonine (143) and solanigroside $\mathrm{P}(\mathbf{1 5 6})$ with three sugar units and $\alpha$-L-rhamnose at C-2 or a hydroxyl group on the steroidal backbone may be potential candidates for the treatment of gastric cancer [228].

Featured here are steroidal pseudoalkaloid oligoglycosides, robeneosides A (153) and B (154) and lobofrutoside (155) from the fruits of $S$. lycocarpum $[182,447]$, and a rare $16 \beta$-H steroidal alkaloid (157) from aerial parts of $S$. surattense [305]. Also included are leptinine I (171) and II (172), the solanidane alkaloid glycosides, isolated from aerial parts of S. orbignianum [46].

Two rare C-3 amino steroidal alkaloids, 188 and 189, were isolated from aerial parts of $S$. triste $[362,471]$. Three C-27 steroidal glycoalkaloids, spiralosides A (194), B (193), C (192), were obtained from the fruits of S. spirale [474]. Esculeoside A (197), a tomato saponin, is a significant component of ripened tomatoes isolated by Toshihiro et al. [475].
Various bioactivities e.g. antibacterial [80, 384, 403, 406, 407], anticancer [13, 305, 458], antidiabetic [182, 183], antifungal [279], anti-inflammatory [303], CNS depressant [294], leishmanicidal [182, 183], molluscicidal [384, 403, 406, 407], neurotoxicity [106], schistosomicidal [185, 186, 447,457 ], spasmolytic [70] and trypanocidal [185, 186, 447, 457] were highlighted as have been exhibited by steroidal alkaloids of Solanum.

Antioxidant activity of $\mathbf{1 4 5}$ and tomatidine (167) from the berries of $S$. aculeastrum was investigated using DPPH, ABTS and reducing power assays, and the highest inhibition was observed when the two compounds were combined, followed by 145 and 167 [13]. Furthermore, $\mathbf{1 4 5}$ exhibited significant anti-inflammatory activity at doses of $30 \mathrm{mg} / \mathrm{kg}$, with a maximum inhibition of $77.75 \%$ in carrageenan-induced rat paw edema, comparing to indomethacin $(81.69 \%)$. It also showed stronger (46.79effect in xylene induced ear edema in mice [303]. Intraperitoneal injection of $\mathbf{1 4 5}(25 \mathrm{mgkg})$ significantly delayed latency of hind limb tonic extensor phase in the picrotoxin-induced convulsions, and it also potentiated thiopental-provoked sleep in a dose-dependent manner [294]. Moreover, 145 exhibited not only the antibacterial activity against Klebsiella and Staphylococcus spp. at concentration of $1 \mathrm{mg}$, together with 139 and 141 [403], but also a potent stemness and invasion inhibitory effect on human colorectal cancer HCT116 cells [155]. Colony Spheroid formation assay showed that solasodine dosedependently prohibited HCT116 cell stemness. CD133, CD44, Nanog, Oct-4 and Sox-2 were inhibited by 145 to reverse stemness and similar mechanism was stimulated in vivo. Transwell and scratch wound assays revealed that 145 impeded HCT116 cell invasion and migration potential strengthened by TGF- $\beta 1$. Moreover, solasodine attenuated TGF- $\beta 1$-induced EMT and decreased MMPs while in vivo study showed the same trend. The results of this study implied that $\mathbf{1 4 5}$ may be a novel therapeutic drug for CRC treatment [155].

Burger et al. documented that the crude extract and aqueous fraction containing 139 displayed potent nonselective cytotoxicity ( $\left.\mathrm{IC}_{50} 15.62 \mu \mathrm{gmL}\right)$ and noteworthy 9.1-fold P-glycoprotein inhibition at $100 \mu \mathrm{gmL}$ [15]. Zhang et al. assessed the molecular mechanism underlying the anti-cancer effect of $\mathbf{1 3 9}$ in human cholangiocarcinoma QBC939 cells. The results revealed that 139 inhibited the viability of QBC939 cells in a dose-dependent manner. Furthermore, it significantly induced the apoptosis of QBC939 cells and altered the mitochondrial membrane potential of cells. Quantitative polymerase chain reaction analysis revealed that $\mathbf{1 3 9}$ decreased the mRNA level of B cell lymphoma-2 (Bcl-2) Bcl-extra-large and X-linked inhibitor of apoptosis protein but increased the mRNA level of Bcl-2-associated X protein (Bax) In addition, 


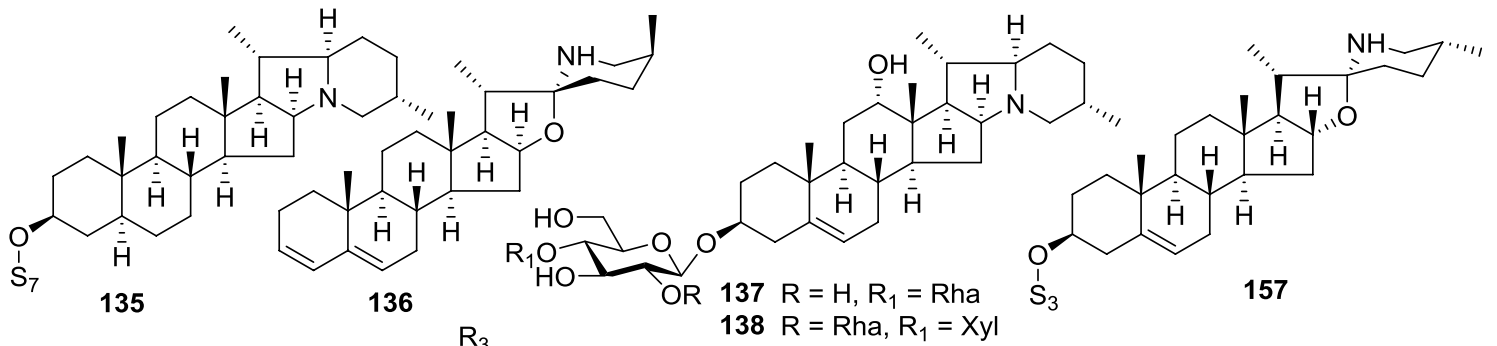

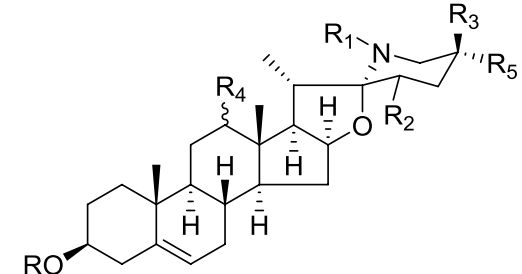

$139 \mathrm{R}=\mathrm{S}_{3}, \mathrm{R}_{1}=\mathrm{R}_{2}=\mathrm{R}_{4}=\mathrm{R}_{5}=\mathrm{H}, \mathrm{R}_{3}=\mathrm{CH}_{3}$

$140 \mathrm{R}=\mathrm{Glc}, \mathrm{R}_{1}=\mathrm{R}_{2}=\mathrm{R}_{4}=\mathrm{R}_{5}=\mathrm{H}, \mathrm{R}_{3}=\mathrm{CH}_{3}$

$141 \mathrm{R}=\mathrm{S}_{6}, \mathrm{R}_{1}=\mathrm{R}_{2}=\mathrm{R}_{4}=\mathrm{R}_{5}=\mathrm{H}, \mathrm{R}_{3}=\mathrm{CH}_{3}$

$142 \mathrm{R}=\mathrm{S}_{5}, \mathrm{R}_{1}=\mathrm{R}_{2}=\mathrm{R}_{4}=\mathrm{R}_{5}=\mathrm{H}, \mathrm{R}_{3}=\mathrm{CH}_{3}$

$143 R=S_{8}, R_{1}=R_{2}=R_{4}=R_{5}=H, R_{3}=C_{3}$

$144 \mathrm{R}=\mathrm{S}_{5}, \mathrm{R}_{1}=\mathrm{R}_{2}=\mathrm{R}_{5}=\mathrm{H}, \mathrm{R}_{3}=\mathrm{CH}_{3}, \mathrm{R}_{4}=\beta \mathrm{OH}$

$145 \mathrm{R}=\mathrm{R}_{1}=\mathrm{R}_{2}=\mathrm{R}_{4}=\mathrm{R}_{5}=\mathrm{H}, \mathrm{R}_{3}=\mathrm{CH}_{3}$

$146 \mathrm{R}=\mathrm{H}, \mathrm{R}_{1}=\mathrm{OH}, \mathrm{R}_{2}=\mathrm{R}_{3}=\mathrm{R}_{4}=\mathrm{H}, \mathrm{R}_{5}=\mathrm{CH}_{3}$

$147 \mathrm{R}=\mathrm{Ac}, \mathrm{R}_{1}=\mathrm{R}_{2}=\mathrm{R}_{4}=\mathrm{R}_{5}=\mathrm{H}, \mathrm{R}_{3}=\mathrm{CH}_{3}$

$148 \mathrm{R}=\mathrm{Glc}, \mathrm{R}_{1}=\mathrm{OH}, \mathrm{R}_{2}=\mathrm{R}_{4}=\mathrm{R}_{5}=\mathrm{H}, \mathrm{R}_{3}=\mathrm{CH}_{3}$

$149 \mathrm{R}=\mathrm{S}_{1}, \mathrm{R}_{1}=\mathrm{R}_{2}=\mathrm{R}_{4}=\mathrm{R}_{5}=\mathrm{H}, \mathrm{R}_{3}=\mathrm{CH}_{3}$

$150 \mathrm{R}=\mathrm{S}_{2}, \mathrm{R}_{1}=\mathrm{R}_{2}=\mathrm{R}_{4}=\mathrm{R}_{5}=\mathrm{H}, \mathrm{R}_{3}=\mathrm{CH}_{3}$

$151 \mathrm{R}=\mathrm{S}_{4}, \mathrm{R}_{1}=\mathrm{R}_{2}=\mathrm{R}_{4}=\mathrm{R}_{5}=\mathrm{H}, \mathrm{R}_{3}=\mathrm{CH}_{3}$

$152 \mathrm{R}=\mathrm{S}_{3}, \mathrm{R}_{1}=\mathrm{R}_{2}=\mathrm{R}_{4}=\mathrm{R}_{5}=\mathrm{H}, \mathrm{R}_{3}=\mathrm{CH}_{2} \mathrm{OH}$

$153 \mathrm{R}=\mathrm{S}_{3}, \mathrm{R}_{1}=\mathrm{R}_{2}=\mathrm{R}_{5}=\mathrm{H}, \mathrm{R}_{3}=\mathrm{CH}_{3}, \mathrm{R}_{4}=\alpha \mathrm{OH}$

$154 \mathrm{R}=\mathrm{S}_{5}, \mathrm{R}_{1}=\mathrm{R}_{2}=\mathrm{R}_{5}=\mathrm{H}, \mathrm{R}_{3}=\mathrm{CH}_{3}, \mathrm{R}_{4}=\alpha \mathrm{OH}$

$155 \mathrm{R}=\mathrm{S}_{3}, \mathrm{R}_{1}=\mathrm{R}_{2}=\mathrm{R}_{5}=\mathrm{H}, \mathrm{R}_{3}=\mathrm{CH}_{3}, \mathrm{R}_{4}=\beta \mathrm{OH}$

$156 \mathrm{R}=\mathrm{S}_{6}, \mathrm{R}_{1}=\mathrm{R}_{2}=\mathrm{R}_{5}=\mathrm{H}, \mathrm{R}_{3}=\mathrm{CH}_{3}, \mathrm{R}_{4}=\beta \mathrm{OH}$
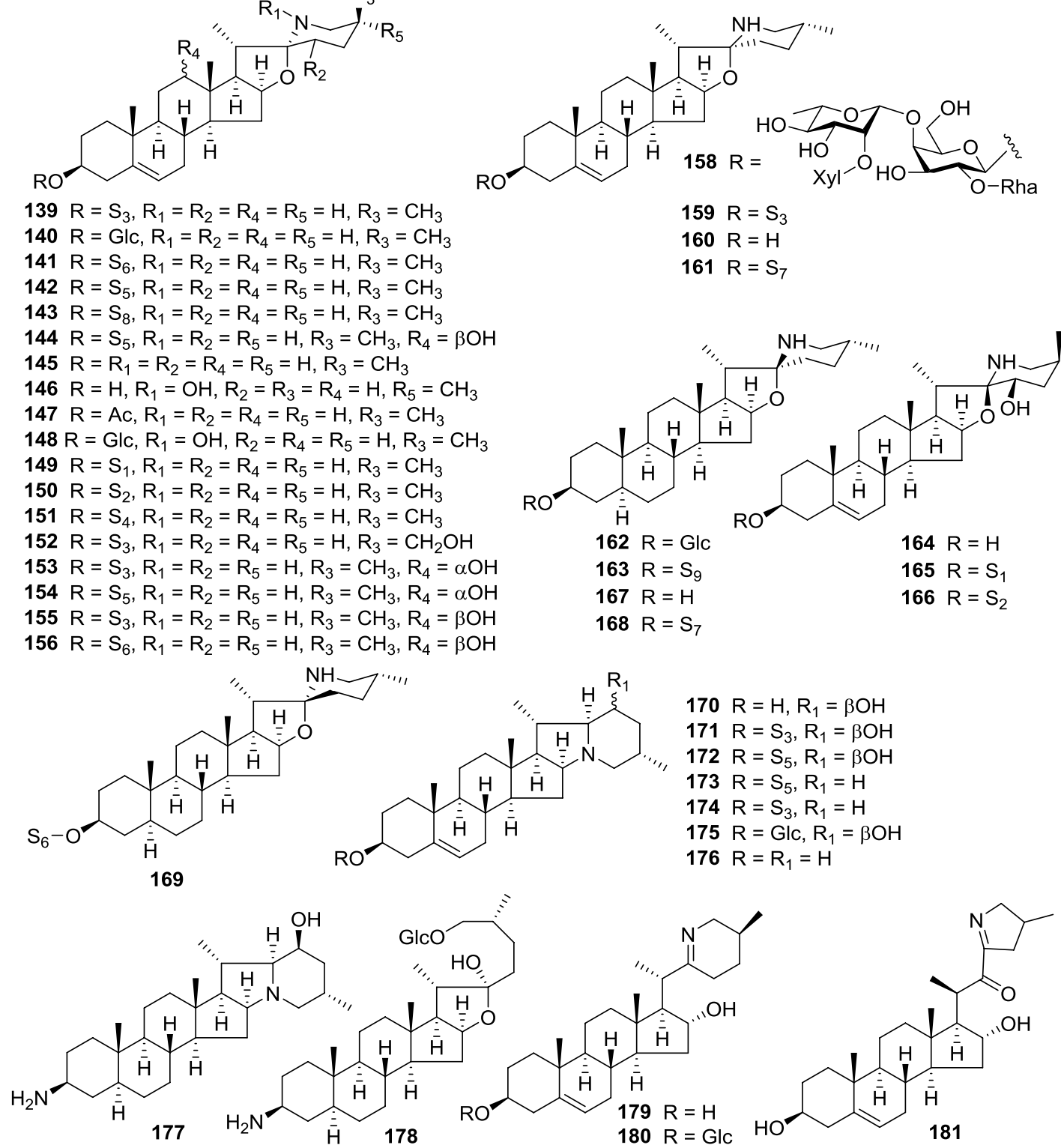

Fig. 2 Steroidal alkaloids 135-197 from Solanum

western blot analysis demonstrated that $\mathbf{1 3 9}$ inhibited the protein expression of $\mathrm{Bcl}-2$ and poly ADP ribose polymerase (PARP) and promoted the protein expression of Bax, cleaved PARP, caspase 3, cleaved caspase 3 and caspase [97].
Compounds 139, 141 and 157 demonstrated cytotoxicity against A549, whereas 139 and $\mathbf{1 5 6}$ showed cytotoxicity against HepG2 cell lines [305]. Compounds $\mathbf{1 3 9}$ and $\mathbf{1 4 1}$ were confirmed as the effective components for Oncomelania snail control. The death rate of Oncomelania snails was 94.2 at a concentration of $2.50 \mathrm{mg} / \mathrm{L} \mathrm{(139)} \mathrm{[406],} \mathrm{while} 141$ 


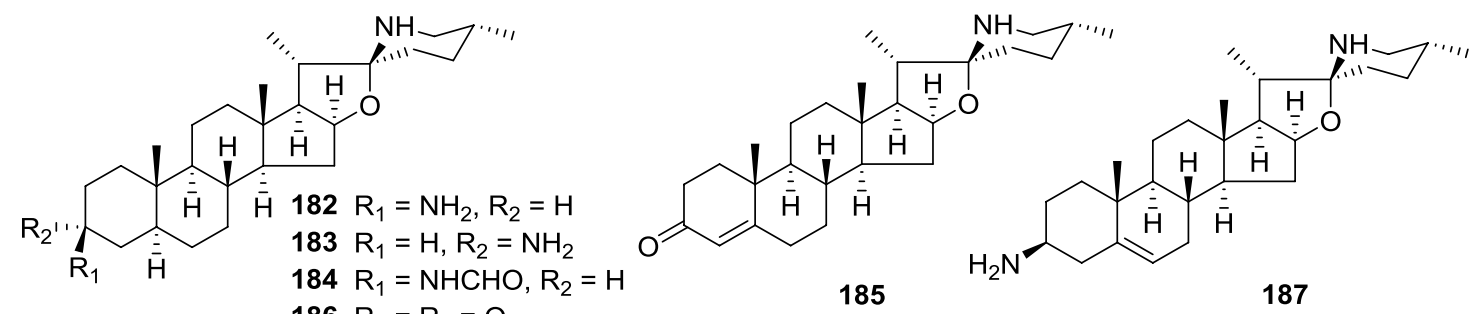

$185 \quad 187$

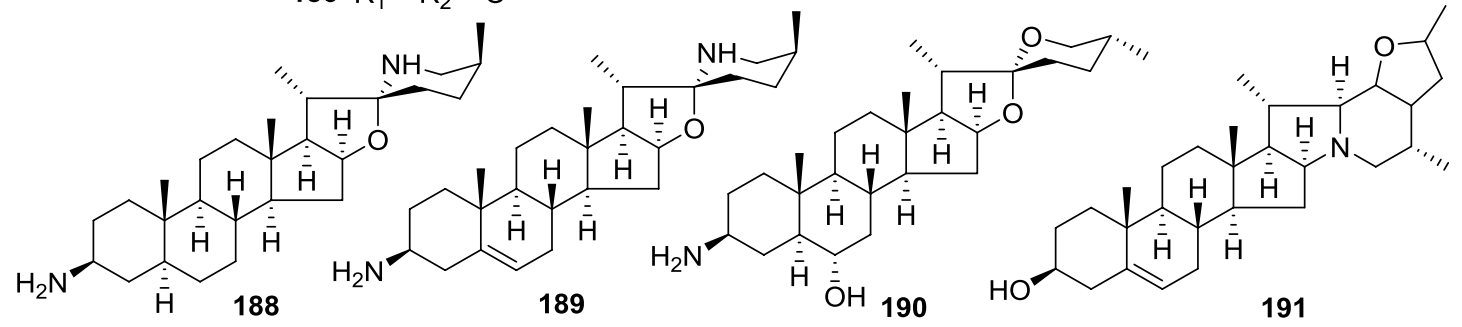

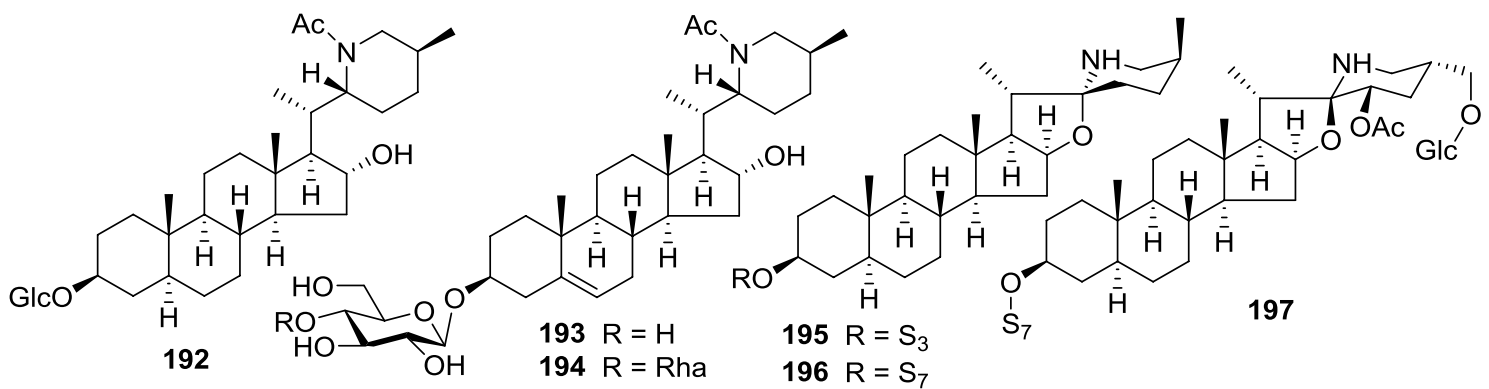

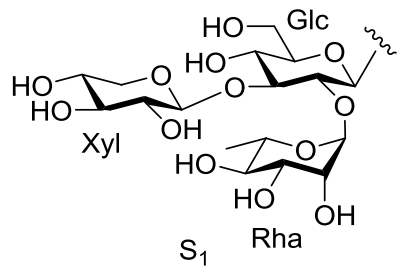

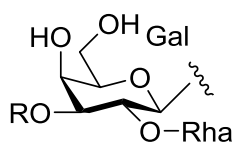

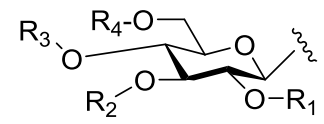

$S_{3} \quad R_{1}=R_{3}=R h a, R_{2}=R_{4}=H$

$\begin{array}{lll}S_{2} R=X y l & S_{4} & R \\ S_{1} & R=R_{3}=R h a, R_{2}=X y l, R_{4}=H\end{array}$

$S_{5} R=G l c \quad S_{6} R_{1}=R_{2}=R_{4}=H, R_{3}=R h a$

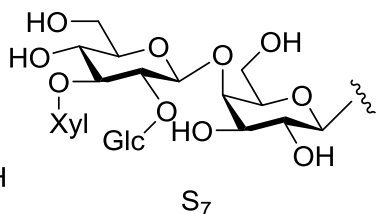

$S_{9} R_{1}=R_{2}=R_{3}=H, R_{4}=X y l$

Fig. 2 (continued)

exhibited a lethality of 100against $O$. hupensis [407]. Moreover, 139 and solasonine (142) displayed not only leishmanicidal activity against promastigote forms of Leishmania amazonensis [185], but also antidiabetic activity by inhibiting the serum glucose increase in oral sucrose-loaded rats and suppressing gastric emptying in mice [182]. A synergistic effect was observed for a mixture of the compounds [183]. Compound 139 also expressed stronger trypanocidal activity $\left(\mathrm{IC}_{50}=15.3 \mu \mathrm{g} / \mathrm{mL}\right.$ ), when compared to benznidazol $\left(\mathrm{IC}_{50}=9.0 \mu \mathrm{g} / \mathrm{mL}\right)$, the only drug used to treat Chagas' disease [186].

Tomatine (168) was illustrated to exert significant neuroprotective effect on $\mathrm{H}_{2} \mathrm{O}_{2}$-induced SH-SY5Y cells, by enhancing intracellular anti-oxidant enzyme activity and brain-derived neurotrophic factor expression and restraining $\mathrm{H}_{2} \mathrm{O}_{2}$-induced oxidative stress [106]. Isojuripidine (190) displayed spasmolytic activity by hindering phasic contractions induced by both histamine and acetylcholinein guineapig ileum [69].

\subsection{Pregnane Glycosides}

Compounds 198-210 from Solanum comprise pregnane glycosides (Fig. 3). These compounds coexist in small amounts and could be biosynthesised from steroidal glycosides [194]. Solanigrosides A (198), B (199), 200 and hypoglaucin H (202) were isolated from S. nigrum [476]. Aerial parts of $S$. torvum gave the highest number of pregnane glycosides, torvpregnanosides A (205) and B (207), ganaxolone (208), allopregnanolone (209) and pregnanolone (210). The whole plant of S. lyratum afforded compounds 203 and 204 [194].

Pregnane glycosides have reportedly demonstrated anticancer properties [194, 317]. Compound 203 exhibited substantial cytotoxic activity against A375-S2, HeLa, SGC7901, and Bel-7402 cell lines, with $\mathrm{IC}_{50}$ values of 13.1 to $49.8 \mu \mathrm{g} / \mathrm{mL}$ [194]. Compound 206 indicated cytotoxicity against human melanoma A375 $\left(\mathrm{IC}_{50}=39.66 \mu \mathrm{M}\right)$ [317]. 

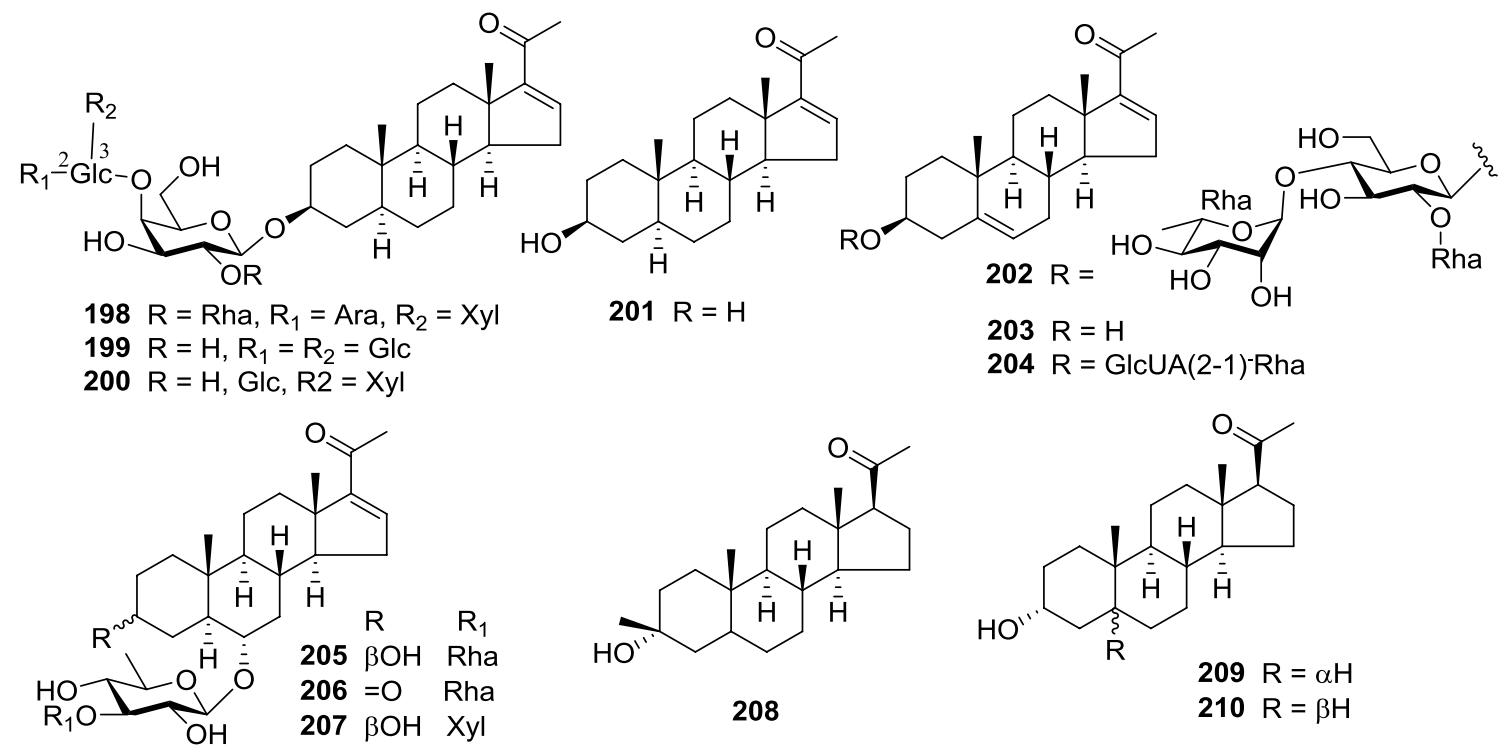

Fig. 3 Pregnane glycosides 198-210 from Solanum

\subsection{Triterpenes}

Fourteen triterpenes (211-224) were identified in Solanum spp. (Figure 4), with lupeol (212) from S. cathayanum [472, 473, 477], S. schimperianum [278], S. spirale [297] and ursolic acid (216) from S. lyratum [197], S. torvum [463] and S. xanthocarpum [427], as the major ones. Six triterpenes 216-217 and 221-224 were reported from the aerial parts of $S$. torvum $[314,463]$. Two cycloartane triterpenoids, cycloeucalenone (213) and 24-oxo-31-norcycloartanone (214) are the main constituents of $S$. cernuum leaves [107]. Daturaolone (218) was isolated for the first time from $S$. arundo [65].

Solanum triterpenes have indicated to possess anticancer properties. For instance, $\mathbf{2 1 3}$ presented significant activity against KB-Oral cavity cancer $\left(\mathrm{IC}_{50}=26.73\right.$ $\mu \mathrm{gmL})$ [297], while $\mathbf{2 1 3}$ exhibited selective activity against lung tumor cell line (NCIH460). The anti-nociceptive activity observed for $\mathbf{2 1 3}$ and $\mathbf{2 1 4}$ was found to be related to the inhibition of different mediators involved in inflammation and nociceptive process. Both compounds decreased cyclooxygenase $2(\mathrm{COX}-2)$ protein expression, although only 214 reached a significant response $(P<0.05$ vs control) [107].

\subsection{Diterpenes}

Four diterpenes, e.g., phytol (225) from S. pseudocapsicum [263], kaur-16-ene (226) from S. aculeastrum [11], solanerioside A (227) from S. erianthum [138], and tricalysioside U (228) from S. violaceum [392] were reported from Solanum spp. (Figure 5). Solanerioside A (227) was the first example of a diterpenoid glucoside featuring a 14, 15-dinor-cyclophytane scaffold [138].

\subsection{Sesquiterpenes}

Sesquiterpenes, 229-310, have been characterized from Solanum spp. (Figure 6). Majority of these compounds, 260-282, were from S. lyratum [196, 197, 199, 200, 484-486] and S. septemlobum [281, 482, 483]. Likewise, 283-285 and 298-303 were reported from S. septemlobum [281, 482, 483]. Compounds 229-231 and 245-255 were isolated from the leaves and fruits of S. erianthum [138, 481], while 286-293 were from the roots of $S$. torvum [487]. Compounds 236-239 were isolated from the roots of S. aethiopicum [29], while 240-242 were obtained from the leaves of S. aculeastrum [11]. The fruits of S. betaceum yielded compounds 306-310 [77].

The bioactivities notedly displayed by sesquiterpenes include anticancer [197-200, 281, 484] and antifungal [3]. 3- $\beta$-Hydroxysolavetivone (232), solavetivone (233) and lubimin (235) from the roots of $S$. abutiloides exhibited anti-fungal activities against Fusarium oxysporum f. sp. Melongenae [3]. The eudesmane-type, solajiangxin D (276), and vetispirane-type, solajiangxin E (277) from $S$. lyratum demonstrated crucial cytotoxicities $\left(\mathrm{ED}_{50}=2.1-3.7 \mu \mathrm{g} / \mathrm{mL}\right)$ against three human cancer lines (P-388, HONE-1, and HT-29) [200]. Solajiangxin B (258), A (274) and C (275) from the whole plant of $S$. lyratum [198] and Septemlobin D (259), and 11,12-O-isopropylidene solajiangxin $F$ (298) [483] also showed significant cytotoxicities $\left(\mathrm{ED}_{50}=1.9-3.7\right.$, and 3.0-7.3 $\mu \mathrm{M}$, resp.) 


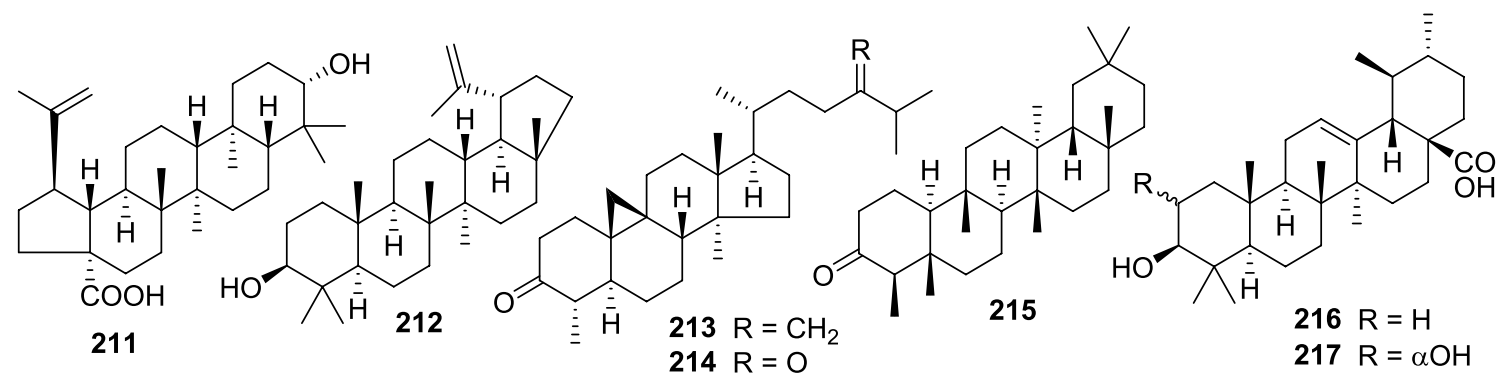

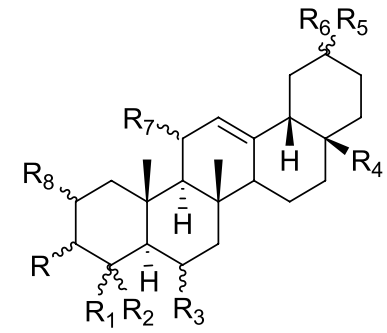

Succinate $=\mathrm{HOOC} \Upsilon \mathrm{COO}-\xi$

$218 R==O, R_{1}=R_{2}=R_{4}=R_{5}=R_{6}=C_{3}, R_{3}=\beta O H, R_{7}=R_{8}=H$

219 R=Succinate, $R_{1}=R_{2}=R_{4}=C_{3}, R_{3}=R_{8}=H, R_{5}=\alpha C_{3}, R_{6}=C O O H, R_{7}==0$

$220 \mathrm{R}=\beta \mathrm{OH}, \mathrm{R}_{1}=\mathrm{R}_{2}=\mathrm{R}_{4}=\mathrm{R}_{5}=\mathrm{R}_{6}=\mathrm{CH}_{3}, \mathrm{R}_{3}=\mathrm{R}_{7}=\mathrm{R}_{8}=\mathrm{H}$

$221 \mathrm{R}=\beta \mathrm{OH}, \mathrm{R}_{1}=\mathrm{R}_{2}=\mathrm{R}_{5}=\mathrm{R}_{6}=\mathrm{CH}_{3}, \mathrm{R}_{3}=\mathrm{R}_{7}=\mathrm{R}_{8}=\mathrm{H}, \mathrm{R}_{4}=\mathrm{COOH}$

$222 \mathrm{R}=\beta \mathrm{OH}, \mathrm{R}_{1}=\mathrm{R}_{2}=\mathrm{R}_{5}=\mathrm{R}_{6}=\mathrm{CH}_{3}, \mathrm{R}_{3}=\mathrm{R}_{7}=\mathrm{H}, \mathrm{R}_{4}=\mathrm{COOH}, \mathrm{R}_{8}=\alpha \mathrm{OH}$

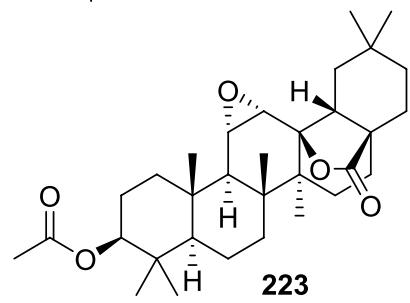

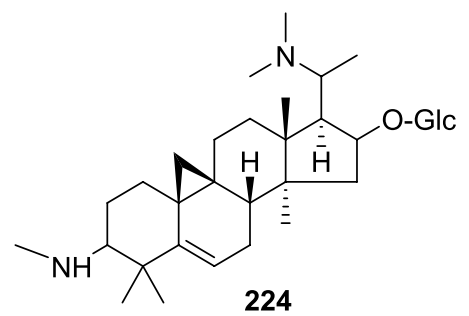

Fig. 4 Triterpenoids 211-224 from Solanum

against these three cancer cell lines. Lyratol D (257), blumenol A (260), dehydrovomifoliol (262) and lyratol C (272) from the whole plant of S. lyratum displayed critical cytotoxic activities against HONE-1 nasopharyngeal, KB oral epidermoid carcinoma, and HT29 colorectal carcinoma cells $\left(\mathrm{IC}_{50}=3.7-8.1 \mu \mathrm{M}\right)$ [199].

Eudesmane-related sesquiterpenes, septemlobins A (301) and B (302) and vetispirane-type, septemlobin $\mathrm{C}(\mathbf{3 0 3})$ exhibited significant cytotoxicities against three cancer cell lines (P-388, HONE-1, and HT-29) $\left(\mathrm{IC}_{50}=3.8-7.5 \mathrm{mM}\right)[281]$.

\subsection{Monoterpenes}

Twenty-eight monoterpenes (311-338) have been characterized from Solanum spp. (Fig. 7), with $\beta$-Ionone (320) reported from S. aculeastrum [11], S. pseudocapsicum [263] and S. betaceum [77], and loliolide (323) obtained from S. erianthum [137], S. americanum [49] and S. pseudocapsicum [263], as dominant monoterpenes. Majority of the compounds, 316-318 and 324-333 [468, 489-492], were obtained from the fruits of $S$. vestissimum. Hotrienol (324), with very sweet and flowery flavor is a well-known constituent of the leaf oil of Cinnamomum camphora. It has also been found in a large number of other natural tissues, such as tea, grapes, wines passion fruit, elderberry flowers, Achillea ligustica and papaya fruit [468]. Seven monoterpenes, 311-313 and 319-322 were reported from the leaves of $S$. aculeastrum [11], and glycosides 329-332 were the aroma precursors in S. vestissimum fruit peelings [468, 492].

\subsection{Flavonoids}

Seventy-two flavonoids 339-413 have been identified in the genus Solanum (Fig. 8), with quercetin (340) and kaempferol (351) as the primary flavonoids. Several glycosylated flavonoids, e.g., afzelin (344), astragalin (346), kaempferol 3-O-[apiofuranosyl- $(1 \rightarrow 2)$ ]- $\alpha$-rhamnoside $(347)$ and - $\beta$-galactoside (348) from $S$. cernuum [501], and camelliaside C (352) from $S$. erianthum [137] were obtained. Five kaempferol derivatives 373-377 were reported from S. elaeagnifolium [502]. Moreover, three anthocyanins 361-363 were isolated from the red and purple tubers of S. tuberosum [508], while five anthocyanin rutinosides 364-368 were reported from the fruits of S. betaceum [75, 76]. Anthocyanins are the largest group of water-soluble pigments in the plant kingdom. They are responsible for most red and blue colours in fruits, vegetables, and have been used in the food industry as pigments, owing to their bright attractive colours, high water solubility and 


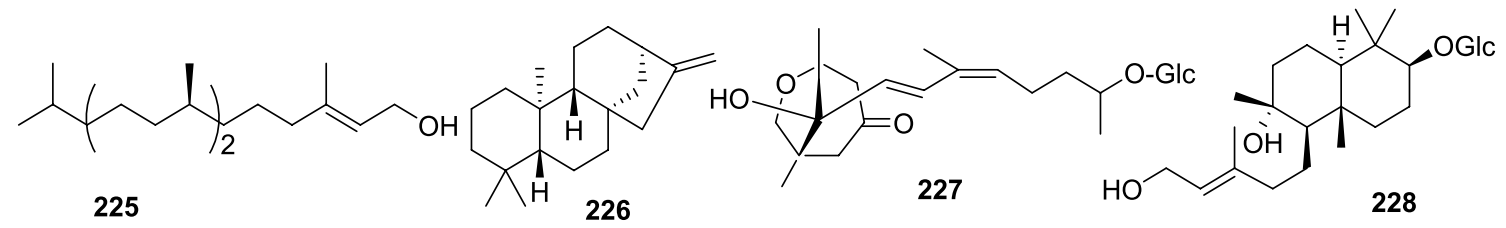

Fig. 5 Diterpenes 225-228 from Solanum<smiles>CC1=CC(=O)CC(C)(C)C1(O)/C=C/C(C)OC1CCCCC1</smiles>

229<smiles>[R]C1CCC[C@@H](C)[C@]12CC[C@@H](C(=C)C)C2</smiles>

$\mathrm{HO}$<smiles>C=CC(C)(O)CC(C=C(C)CCC=C(C)CO)OCl</smiles>

230<smiles>C=C(C)[C@@H]1CC[C@]2(C[C@H](C)C[C@@H](C)CC2=O)C1</smiles>

243 244<smiles>C=C[C@](C)(O)CC/C=C(\C)CC[C@H](O[Cl+3])C(C)(C)O</smiles>

231<smiles>[Z14][C@](C)(O)CC/C=C(/C)CCC=C(C)C</smiles>

$232 \mathrm{R}=\mathrm{OH}, \mathrm{R}_{1}=\mathrm{CH}_{3}$ $233 \mathrm{R}=\mathrm{H}, \mathrm{R}_{1}=\mathrm{CH}_{3}$ $234 \mathrm{R}=\mathrm{H}, \mathrm{R}_{1}=\mathrm{CH}_{2} \mathrm{OH}$

$235 \mathrm{R}=\alpha-\mathrm{CHO}$

$236 \mathrm{R}=\alpha-\mathrm{COOH}$

$237 \mathrm{R}=\beta-\mathrm{CHO}$

$238 \mathrm{R}=\beta-\mathrm{COOH}$

$239 \mathrm{R}=\alpha-\mathrm{CH}_{2} \mathrm{OH}$<smiles>CC1=C2CC(C)CC(C)C2CCC1C</smiles><smiles>CCCC1C(C)CCC(C)C1C</smiles><smiles>C=C(C)C1CC2=C(C(C)CC2)C(C)C1</smiles><smiles>[Z20]C(CCCCC(C)C)CCC(C)CC</smiles><smiles>C=C(C)C1CCC2(C1)C(C)=CC(=O)C=C2C</smiles>
245<smiles></smiles>

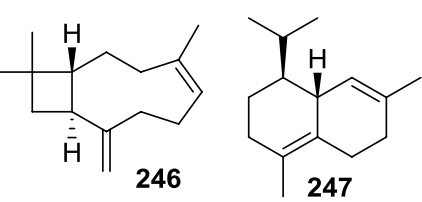<smiles>CC(C)=C1CCC(C(C)C)c2cc(C)ccc21</smiles><smiles>C=C1CCC[C@]2(C)CC[C@@H](C(C)(C)O)C[C@@H]12</smiles><smiles>CC1(C)CC2C3CCC4(CCC(O)C34)C2C1</smiles><smiles>CC1(C)CC=CC2(C)C1=CCCC2(C)C</smiles>

254<smiles>C=C(C)[C@@H]1CC[C@@H](C)C2=C(C1)[C@H](C)CC2</smiles><smiles>[R]Cc1cc([R1])cc([R2])c1C[C@H]1CC(=O)OC1(C)C</smiles>
$256 \mathrm{R}=\mathrm{R}_{1}=\mathrm{H}, \mathrm{R}_{2}=\mathrm{CH}_{3}$

259

253

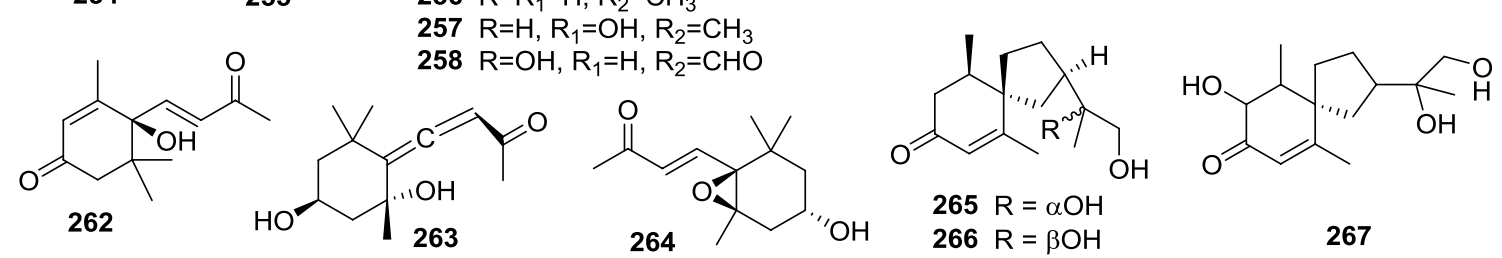

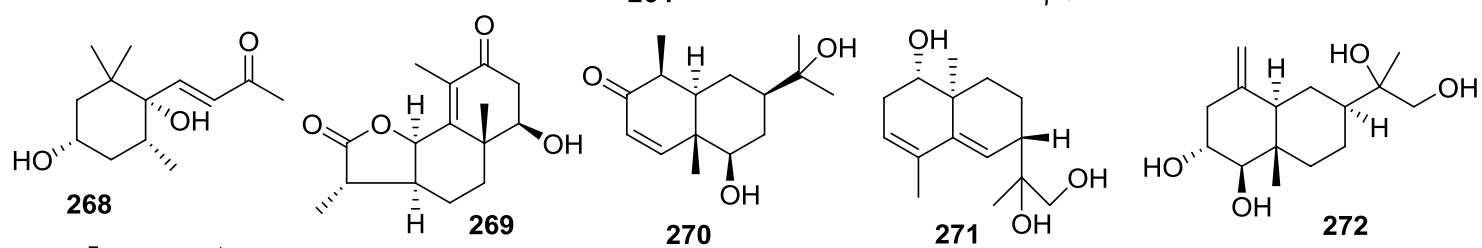<smiles>[Z10]C1=CC(=O)[C@H](O)[C@]2(C)CC[C@H](C(C)(C)O)C[C@]12O</smiles>

270

$271 \mathrm{OH}$<smiles>CC1=CC(=O)CC(C)(C)[C@H]1CC[C@H](C)O</smiles>

260

274

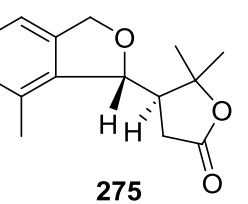<smiles>C=C1C[C@@H](O)[C@H](O)[C@]2(C)CC[C@H](C(C)C(=O)O)C[C@H]12</smiles><smiles>[R7][Y]</smiles>

Fig. 6 Sesquiterpenes 229-310 from Solanum 
<smiles>CC[C@H]1CC[C@@]2(C)C=CC(=O)C(C)=C2C[C@@H]1C(C)(O)CO</smiles>

278<smiles>C=C1CCC[C@]2(C)C=C3OC(=O)C(C)=C3C[C@H]12</smiles>

282<smiles>CC1=CC(=O)C=C(C)C12CCC(C(C)(O)CO)C2</smiles>

288<smiles>CC1=C2C[C@H](C(C)CO)[C@@H](O)C[C@]2(C)C=CC1=O</smiles>

279<smiles>CC1=CC(=O)C[C@H](C)[C@]12CC[C@@](O)(C1COC(C)(C)O1)C2</smiles>

280<smiles>CC(C)(O)[C@@H]1CC[C@@]2(C)C=CC(=O)C=C2C1</smiles>

281<smiles>C=C(C)[C@@H]1CC[C@H]2C(=C(C)C(=O)C[C@H]2O)[C@@H]1O</smiles><smiles>C=C1C[C@@H](O)[C@@H](O)[C@]2(C)CC[C@@H](C(=C)C)C[C@@H]12</smiles>

284<smiles>CC(=O)C1=C[C@]2(CC1)C(C)=CC(=O)C[C@H]2C</smiles><smiles>[R]C1C[C@]2(C)CC[C@@H](C(C)(C)O)C[C@H]2[C@@](C)(O)C1[R]</smiles>

285

$286 \mathrm{R}=\alpha-\mathrm{OH}, \mathrm{R}_{1}=\mathrm{H}$ $287 \mathrm{R}=\mathrm{H}, \mathrm{R}_{1}=\beta-\mathrm{OH}$
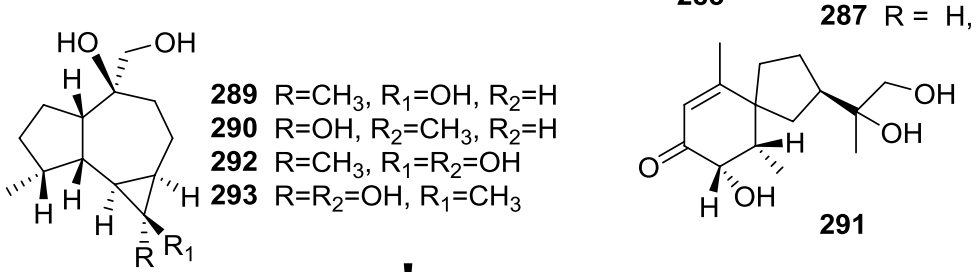<smiles>C=C1C=CCC(C)(C)C12CCC(C)O2</smiles><smiles>[R]c1ccc(C(=O)O[C@@H]2CC(C)=CC[C@]3(C)CC[C@](O)(C(C)C)[C@H]23)cc1[R2]</smiles><smiles>[R7]CC[C@@H]([C@H]1CC=C(C)CC1)[C@@](C)(O)CCC=C(C)C</smiles><smiles>CC1=C2CC([C@H]3COC(C)(C)O3)CCC2CC1=O</smiles><smiles>CC(C)[C@@H]1CC[C@]2(C)CCC[C@H](C)[C@H]2C1</smiles><smiles>[R]c1cccc(C)c1C[C@@H]1CC(=O)OC1(C)C</smiles><smiles>C=C(C)[C@H]1CC[C@@]2(C1)C(C)=CC(=O)C[C@H]2C</smiles><smiles>CC(C)(O)C1CC[C@@]23CC(C(C)(C)O)CCC24CC[C@@H](O)[C@](C)(O4)[C@H]3C1</smiles>

$294 \mathrm{R}_{1}=\mathrm{R}_{2}=\mathrm{H}$

$295 \mathrm{R}_{1}=\mathrm{OCH}_{3}, \mathrm{R}_{2}=\mathrm{OH}$
$296 \mathrm{R}_{1}=\mathrm{H}, \mathrm{R}_{2}=\mathrm{OH}$<smiles>CC1=CC[C@]2(C)CCCC(C)(C)[C@@]23CC13</smiles>

306

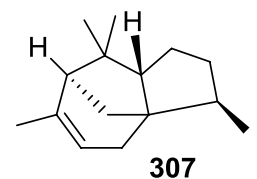

303

304
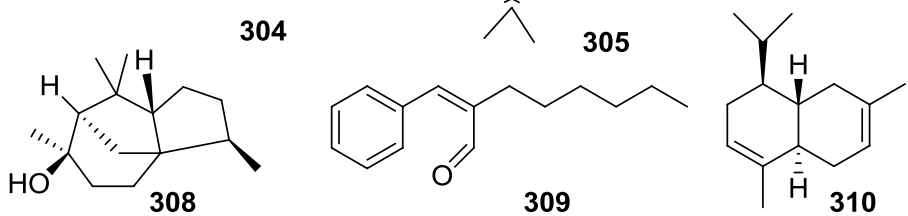

Fig. 6 (continued)

associated health benefits [76]. In addition, diverse flavonoids, such as 388-397 from $S$. jabrense [167] and $S$. palodusum [513] and 399-403 from S. lyratum [514] were reported.

Flavonoids of Solanum have displayed various biactivities e.g., anticancer [31, 75, 76, 503], anti-depressant and antiviral [322, 332] and hepatoprotective [502] characteristics. Compound $\mathbf{3 7 3}$ exhibited significant hepatoprotective and curative effects against histopathological and histochemical damage induced by paracetamol in liver [502], while $\mathbf{3 4 9}$ and $\mathbf{3 7 1}$ displayed cytotoxicity against breast MCF7 and liver HPG2 cancer cell lines [503].

Compound 340 and rutin (342) indicated potent and concentration-dependent free radical-scavenging activity [45]. They also inhibited peroxidation of cerebral and hepatic lipids subjected to iron oxidative assault. Compound 340 induced in vitro antiproliferative and apoptotic activities on Jurkat cells $\left(\mathrm{IC}_{50}=11.77 \pm 2.4 \mathrm{mg} / \mathrm{mL}\right.$ ) [23], while 364-367 showed antioxidant activities [75]. Torvanol A (409) from the roots of $S$. torvum exhibited antidepressant, anxiolytic and adaptogenic effects [316], as well as anti-HSV-1 activity $\left(\mathrm{IC}_{50}=9.6 \mu \mathrm{gmL}\right)$ [322].

\subsection{Lignans}

Lignans, widely distributed in the plant kingdom, are a family of secondary metabolites produced by oxidative dimerization of two phenylpropanoid units. Although their molecular scaffold consists only of two phenylpropane (C6-C3) units, lignans exhibit an enormous structural diversity originating from various linkage patterns of these phenylpropane units. As the C-8-C-3'/C-7-O-C-4' linked lignans containing two chiral centers (C-7 and C-8) comprise the core of 2, 3-dihydrobenzo[b]furan [480].

Lignans are rare in the genus Solanum [79], with only 31 compounds (414-444) having been isolated (Fig. 9). Compounds 414-419 were obtained from the stems of $S$. buddleifolium [79], while $\mathbf{4 2 4 - 4 3 2 , 4 3 4}$ and $\mathbf{4 4 2}$ were isolated from the roots of S. melongena [208-210]. Several neo-lignans, sisymbrifolin (433) from the fruits of S. sisimbriifolium 


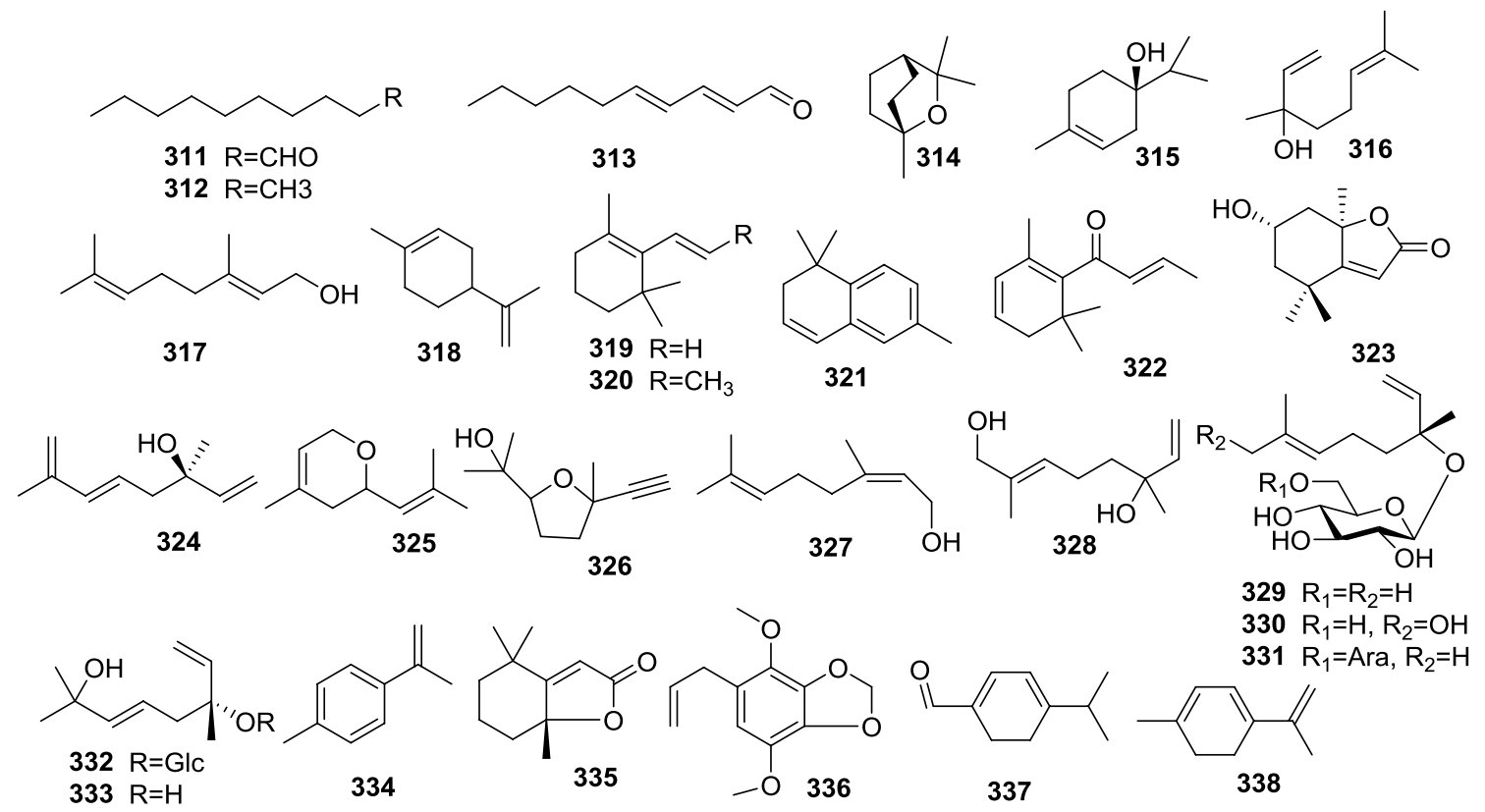

Fig. 7 Monoterpenes 311-338 from Solanum

[519], ficusal (442) from the roots of S. melongena [209], glycosmisic acid (439), simulanol (440) and balanophonin (443) from the whole plant of $S$. surattense [518] were identified. A pair of new C-8-C-3'/C-7-O-C-4' linked neolignan enantiomers, $\mathbf{4 2 0}$ and 421, were isolated from the stems of S. erianthum [480]. Lignanamides 424-432 and 434 were obtained from the roots of $S$. melongena [210].

Among lignans from the genus Solanum, only lignanamides (425-432) were reported with bioactivities. They displayed anti-inflammatory activities by inhibition of nitric oxide production in lipopoly-saccharide-induced RAW 264.7 macrophages $\left(\mathrm{IC}_{50}=16.2\right.$ to $\left.58.5 \mu \mathrm{M}\right)$ [210].

\subsection{Other Alkaloids}

The alkaloids have a natural (2-aminopyrrolidin-1-yl) carboxamidine alkaloidal base acylated with isoferulic (3-hydroxy-4-methoxycinnamic) acid with $\mathrm{Z}$ and $\mathrm{E}$ configurations, resp. [111]. Thirty-one alkaloids $\mathbf{4 4 5 - 4 7 5}$ have been isolated from Solanum spp. (Fig. 10), comprising types of cyclic guanidine alkaloids, e.g., cernumidine (446) and isocernumidine (447) from the leaves of S. cernuum [109, 111, 112]. Bioactive long chain amides, 454-456, exhibiting antimicrobial activity against Escherichia coli and Candida albicans were isolated from aerial parts of S. schimperianum [277]. Compounds 472-474 were obtained from S. sessiliflorum [525].

Antidiabetic activity was illustrated by Solanum alkaloids [49, 209]. Four amides, $N$-trans- $p$-coumaroyl -octopamine (464) and -tyramine (466), and $N$-trans- $p$-feruloyl -octopamine (465) and -tyramine (467) exhibited antidiabetic properties by enhancing $\alpha$-glucosidase inhibitory activity in a study involving dual high-resolution $\alpha$-glucosidaseradical scavenging inhibition profiling [35]. Moreover, 459, 466 and 468 demonstrated possession of inhibitory activity against $\alpha$-glucosidase $\left(\mathrm{IC}_{50}=500.6,5.3\right.$ and $46.3 \mu \mathrm{M}$, resp.) [209].

\subsection{Sterols}

Sixty-six sterols (476-541) were obtained from the genus Solanum (Fig. 11), with $\beta$-sitosterol (483), daucosterol (484) and stigmasterol (485) as the main sterol constituents. Clistol G (476) and capsisteroids A-F (477-482) were obtained from the leaves of $S$. capsicoides [85], tumacones A (507) and B (508) and tumacosides A (509) and B (510) were from the leaves of S. nudum [242-247], carpesterol (517) was isolated from the seeds of $S$. capsicoides [86], and its derivatives (518-521) were reported from the fruits of $S$. xanthocarpum [401]. From the seeds of S. elaeagnifolium, 491, 495, 496 and 498 were yielded [134]. Additionally, two 26-aminochole- stane-type glycosides, abutilosides A (528) and B (529), and five 26-hydroxycholestane-type glycosides, abutilosides C-G (534-538), were isolated from the fresh roots of $S$. abutiloides [5-9]. These compounds are important intermediates in the biogenesis of steroidal alkaloids [5].

Sterols in Solanum have indicated possession of anticancer [86], antifungal [401], and antiplasmodial [242, 245, 247] features. For instance, $\mathbf{5 0 9}$ and $\mathbf{5 1 0}$ displayed in vitro 
<smiles>[R17]Oc1c(-c2cc([R2])c(O)c([R])c2)oc2cc([R20])cc(O)c2c1=O</smiles>

$339 \mathrm{R}=\mathrm{R}_{1}=\mathrm{Me}, \mathrm{R}_{2}=\mathrm{OMe}, \mathrm{R}_{3}=\mathrm{OH}$

$340 \mathrm{R}=\mathrm{R}_{1}=\mathrm{R}_{2}=\mathrm{H}, \mathrm{R}_{3}=\mathrm{OH}$

$341 \mathrm{R}=\mathrm{Rha}, \mathrm{R}_{1}=\mathrm{R}_{2}=\mathrm{R}_{3}=\mathrm{H}$

$342 \mathrm{R}=\mathrm{H}, \mathrm{R}_{1}=$ Rutinose, $\mathrm{R}_{2}=\mathrm{H}, \mathrm{R}_{3}=\mathrm{OH}$

$343 \mathrm{R}=\mathrm{Rha}, \mathrm{R}_{1}=$ Rutinose, $\mathrm{R}_{2}=\mathrm{R}_{3}=\mathrm{H}$

$344 \mathrm{R}=\mathrm{H}, \mathrm{R}_{1}=\mathrm{Rha}, \mathrm{R}_{2}=\mathrm{R}_{3}=\mathrm{H}$

$345 \mathrm{R}=\mathrm{H}, \mathrm{R}_{1}=\mathrm{Rha}, \mathrm{R}_{2}=\mathrm{OH}, \mathrm{R}_{3}=\mathrm{H}$

$346 \mathrm{R}=\mathrm{R}_{2}=\mathrm{R}_{3}=\mathrm{H}, \mathrm{R}_{1}=\mathrm{Glc}$,

$347 \mathrm{R}=\mathrm{H}, \mathrm{R}_{1}=$ apiofuranosyl $(1 \rightarrow 2)-\mathrm{Rha}, \mathrm{R}_{2}=\mathrm{R}_{3}=\mathrm{H}$

$348 \mathrm{R}=\mathrm{H}, \mathrm{R}_{1}=$ apiofuranosyl $-(1 \rightarrow 2)-\beta-\mathrm{D}-\mathrm{Gal}, \mathrm{R}_{2}=\mathrm{R}_{3}=\mathrm{H}$

$349 \mathrm{R}=\mathrm{H}, \mathrm{R}_{1}=6$ "-trans-cynamoyl-glu, $\mathrm{R}_{2}=\mathrm{R}_{3}=\mathrm{H}$

$350 \mathrm{R}=\mathrm{H}, \mathrm{R}_{1}=6$ "-cis-cynamoyl-glu, $\mathrm{R}_{2}=\mathrm{R}_{3}=\mathrm{H}$

$351 \mathrm{R}=\mathrm{R}_{1}=\mathrm{R}_{2}=\mathrm{R}_{3}=\mathrm{H}$

$352 \mathrm{R}=\mathrm{H}, \mathrm{R}_{1}=\beta-\mathrm{D}-\mathrm{Gal}-(2 \rightarrow-1)-\beta-\mathrm{D}-\mathrm{Glc}, \mathrm{R}_{2}=\mathrm{R}_{3}=\mathrm{H}$

$353 \mathrm{R}=\mathrm{H}, \mathrm{R}_{1}=\beta$-D-Glucosyl- $(2 \rightarrow 1)-\beta$-D-Glucoside, $\mathrm{R}_{2}=\mathrm{H}, \mathrm{R}_{3}=\mathrm{OH}$

$354 \mathrm{R}=\mathrm{H}, \mathrm{R}_{1}=$ Rutinose, $\mathrm{R}_{2}=\mathrm{H}, \mathrm{R}_{3}=\mathrm{OMe}$

$355 \mathrm{R}=\mathrm{H}, \mathrm{R}_{1}=\mathrm{Glc}, \mathrm{R}_{2}=\mathrm{OMe}, \mathrm{R}_{3}=\mathrm{H}$

$356 \mathrm{R}=\mathrm{Glc}, \mathrm{R}_{1}=\mathrm{R}_{2}=\mathrm{R}_{3}=\mathrm{H}$

$357 \mathrm{R}=\mathrm{H}, \mathrm{R}_{1}=\mathrm{Rha}-(1 \rightarrow 6)-\beta$-D-gal, $\mathrm{R}_{2}=\mathrm{H}, \mathrm{R}_{3}=\mathrm{OH}$

$358 \mathrm{R}=\mathrm{R}_{2}=\mathrm{R}_{3}=\mathrm{H}, \mathrm{R}_{1}=(6$ "-O-cis- $p$-coumaroyl) galactose

$359 \mathrm{R}=\mathrm{H}, \mathrm{R}_{1}=\beta$-D-gal, $\mathrm{R}_{2}=\mathrm{R}_{3}=\mathrm{OH}$

$360 \mathrm{R}=\mathrm{R}_{1}=\mathrm{R}_{2}=\mathrm{R}_{3}=\mathrm{H}$

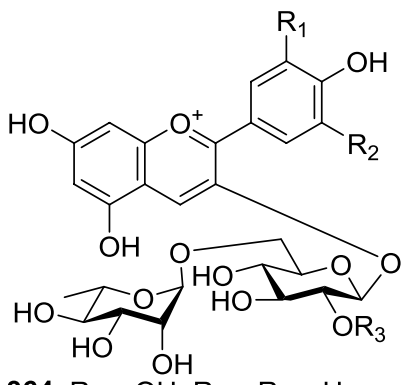

$364 \mathrm{R}_{1}=\mathrm{OH}, \mathrm{R}_{2}=\mathrm{R}_{3}=\mathrm{H}$

$365 R_{1}=R_{2}=R_{3}=H$

$366 \mathrm{R}_{1}=\mathrm{R}_{3}=\mathrm{H}, \mathrm{R}_{2}=\mathrm{OH}$

$367 \mathrm{R}_{1}=\mathrm{OH}, \mathrm{R}_{2}=\mathrm{O}-\mathrm{Glc}, \mathrm{R}_{3}=\mathrm{H}$

$368 \mathrm{R}_{1}=\mathrm{OH}, \mathrm{R}_{2}=\mathrm{H}, \mathrm{R}_{3}=\beta-\mathrm{D}-\mathrm{Xyl}$

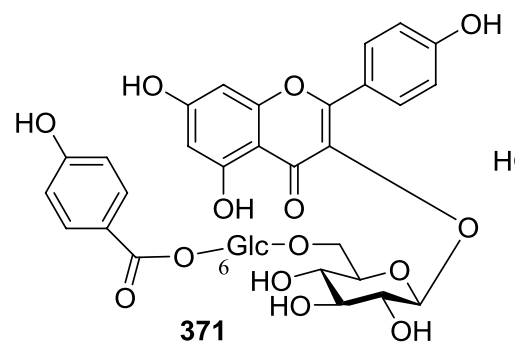<smiles>Oc1ccccc1</smiles><smiles></smiles>

369

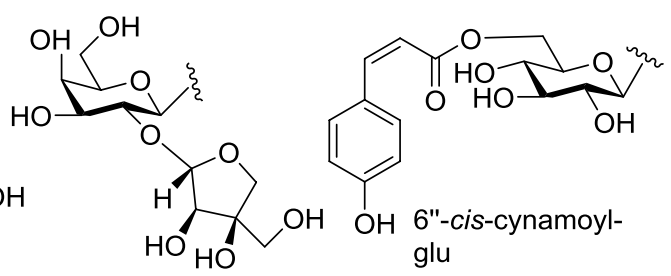

apio(f)l-(1-2)-Gal

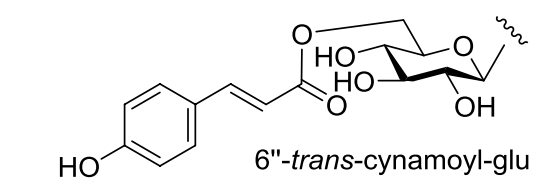

$\mathrm{HO}$<smiles></smiles>

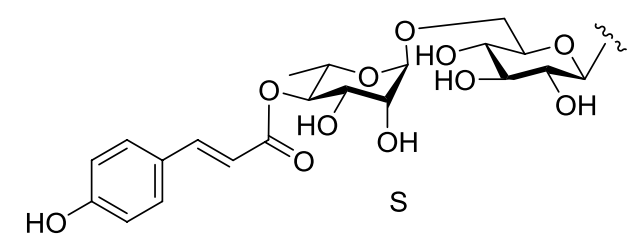

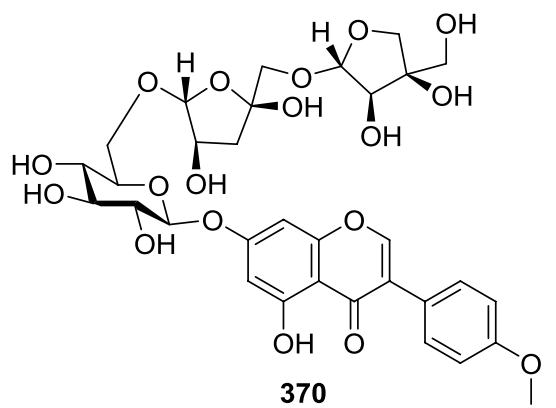

Fig. 8 Flavonoids 339-413 from Solanum

antimalarial activity against $P$. falciparum chloroquineresistant FCB-1 strain $\left(\mathrm{IC}_{50}=27\right.$ and $\left.16 \mu \mathrm{M}\right)$ [247]. Compounds 511-515 from aerial parts of $S$. nudum demonstrated antiplasmodial activity on hepatic trophozoites of $P$. vivax. All the steroids reduced the number of hepatic $P$. vivax trophozoites. Among them, 506 and $\mathbf{5 1 2}$ reduced the number 
<smiles>O=c1c(O)c(-c2ccc(O)c(O)c2)oc2cc(O)c(Cl)c(O)c12</smiles>

378<smiles>[R20]Oc1c(-c2ccc(O)c(O)c2)oc2cc(O)c([R])c(O)c2c1=O</smiles>

$379 R_{1}=H, R_{2}=$ Gal

$380 R_{1}=H, R_{2}=(B-D$-gluco $(f)$

$381 R_{1}=H, R_{2}=\operatorname{Gal}(2-1)$ apio(f)<smiles>[R]c1c(OC)cc(-c2oc3cc(OC)cc(O)c3c(=O)c2[R])c([R])c1OC</smiles>

$382 \mathrm{R}=\mathrm{R}_{2}=\mathrm{H}, \mathrm{R}_{1}=\mathrm{OCH}_{3}$

$383 \mathrm{R}=\mathrm{OMe}, \mathrm{R}_{1}=\mathrm{H}, \mathrm{R}_{2}=\mathrm{OCH}_{3}$<smiles>O=C(O)C1(CO)C(O)C(O)C(O)C(O)C1Oc1cc2oc(-c3ccccc3)cc(=O)c2c(O)c1O</smiles>

384

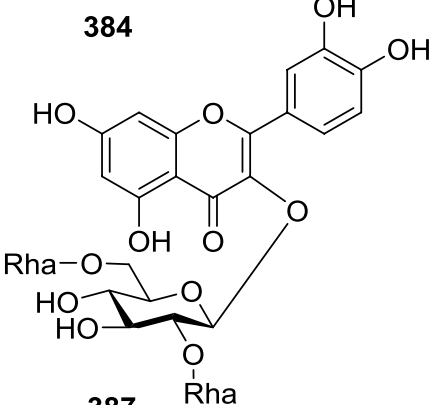

387<smiles>COc1cc(-c2c(-c3ccc(OC(=O)/C=C/c4cc(O)ccc4O)c(OC)c3)oc3cc(O)cc(O)c3c2=O)ccc1O</smiles>

385

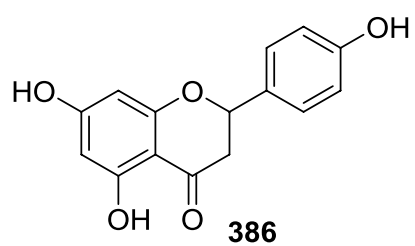<smiles>[R20]c1ccc(-c2coc3cc([R20])cc([R1])c3c2=O)cc1</smiles>

$399 \mathrm{R}=\mathrm{R}_{1}=\mathrm{H}, \mathrm{R}_{2}=\mathrm{CH}_{3}$

$400 \mathrm{R}=\mathrm{Glc}, \mathrm{R}_{1}=\mathrm{H}, \mathrm{R}_{2}=\mathrm{CH}_{3}$

$401 \mathrm{R}=\mathrm{R}_{1}=\mathrm{H}, \mathrm{R}_{2}=\mathrm{H}$

$402 \mathrm{R}=\mathrm{Glc}, \mathrm{R}_{1}=\mathrm{OH}, \mathrm{R}_{2}=\mathrm{H}$

$404 \mathrm{R}=\mathrm{Glc}, \mathrm{R}_{1}=\mathrm{OH}, \mathrm{R}_{2}=\mathrm{CH}_{3}$<smiles>COc1cc(O)cc2oc(O)c(-c3ccc(O)cc3)c(=O)c12</smiles>

404<smiles>COc1ccc2c(c1)C(OS(=O)(=O)O)[C@@H](c1cc(/C=C/C(=O)O)cc(OC)c1O)CO2</smiles><smiles>[R20]Oc1c(-c2ccc([R20])c([R3])c2)oc2cc([R20])cc(O)c2c1=O</smiles><smiles>COc1cc2c(c3oc(-c4ccccc4)cc(=O)c13)C(OC(C)=O)C(C)(C)OC2OC(C)=O</smiles>

Fig. 8 (continued)

of hepatic trophozoites by 47and 39resp. [245]. Compound 517 produced antiproliferative activity in glioma (U251), breast (MCF-7), kidney (786-0), ovary (OVCAR-03), and
K562 cell lineages [86]. In addition, 505-509 displayed antifungal activity by inhibiting radial growth of $A$. niger and T. viride [401]. 
<smiles>[R]c1cc([C@H]2c3c(cc(OC)c(O)c3[R])C[C@@H]([R2])[C@@H]2[R1])cc(OC)c1O</smiles>

$\begin{array}{ccccc} & \mathrm{R} & \mathrm{R}_{1} & \mathrm{R}_{2} & \mathrm{R}_{3} \\ 414 & \mathrm{H} & \alpha \mathrm{CH}_{2} \mathrm{OH} & \beta \mathrm{CH}_{2} \mathrm{OH} & \mathrm{H} \\ 415 & \mathrm{OCH}_{3} & \alpha \mathrm{CH}_{2} \mathrm{OH} & \beta \mathrm{CH}_{2} \mathrm{OH} & \mathrm{H}\end{array}$

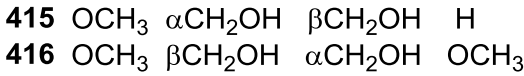

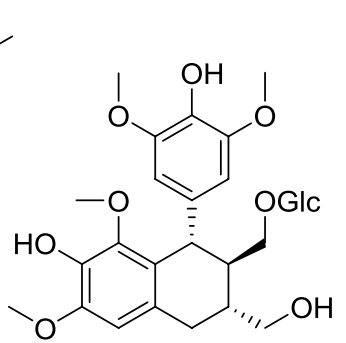
417<smiles>COC[C@H]1C(c2cc(OC)c(O)c(OC)c2)c2c(cc(OC)c(O)c2OC)C[C@@H]1CO</smiles><smiles>COc1cc(C[C@@H]2CO[C@H](c3cc(OC)c(O)c(OC)c3)C2COC(C)=O)cc(OC)c1O</smiles><smiles>[R]C1c2cc(/C=C/COC)cc(OC)c2O[C@H]1c1cc(OC)c(O)c(OC)c1</smiles>

$420 \mathrm{R}=\mathrm{BCH}_{2} \mathrm{OH}$

$421 \mathrm{R}=\alpha \mathrm{CH}_{2} \mathrm{OH}$<smiles>[R7]Oc1c([R])cc([C@@H]2OC[C@H]3[C@H](C)OC[C@@H]32)cc1OC</smiles>

$422 \mathrm{R}=\mathrm{R}_{2}=\mathrm{OCH}_{3}, \mathrm{R}_{1}=\mathrm{R}_{3}=\mathrm{Glc}$

$423 \mathrm{R}=\mathrm{R}_{2}=\mathrm{OCH}_{3}, \mathrm{R}_{1}=\mathrm{R}_{3}=\mathrm{H}$

$435 \mathrm{R}=\mathrm{R}_{1}=\mathrm{R}_{2}=\mathrm{R}_{3}=\mathrm{H}$

$436 \mathrm{R}=\mathrm{R}_{2}=\mathrm{R}_{3}=\mathrm{H}, \mathrm{R}_{1}=\mathrm{Glc}$

$437 \mathrm{R}=\mathrm{OCH}_{3}, \mathrm{R}_{1}=\mathrm{R}_{2}=\mathrm{R}_{3}=\mathrm{H}$<smiles>[R]c1c(O)c(OC)cc2c1C(c1ccc(O)c(OC)c1)C(C(=O)NCC)C(C(=O)NC)=C2</smiles>

$437 \mathrm{R}=\mathrm{R}_{2}=\mathrm{OCH}_{3}, \mathrm{R}_{1}=\mathrm{Glc}, \mathrm{R}_{3}=\mathrm{H}$

$424 \mathrm{R}=\mathrm{OCH}_{3}$<smiles>COc1cc(CO)ccc1CO</smiles><smiles>[R]C(CNC(=O)/C=C/c1cc(OC)c(OC)c(OC)c1)c1ccc(O)cc1</smiles><smiles>[Y]NC(=O)/C(=C/c1ccc(O)c([R])c1)Oc1ccc(/C=C/C(=O)NC)cc1[R]</smiles>

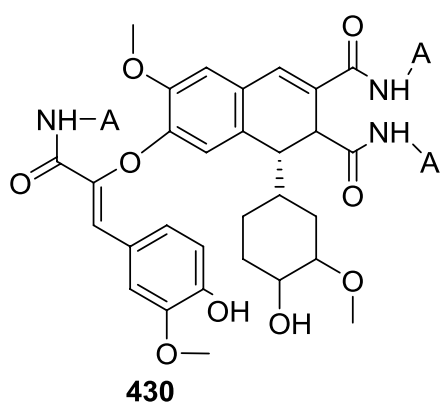<smiles>[R][R](=O)O</smiles><smiles>CCCc1ccc(O)cc1</smiles>

$427 \mathrm{R}=\mathrm{H}$<smiles>[3H]NC(=O)[C@@H]1C(C(N)=O)=Cc2cc(OC)c(O)c(OC)c2[C@H]1c1cc(OC)c(O)c(OC)c1</smiles>

432<smiles>[R]Cc1cc(OC)c2c(c1)[C@H](CO)[C@H](c1cc([R1])c(O)c(OC)c1)O2</smiles>

$439 \mathrm{R}=\mathrm{CHCOOH}, \mathrm{R}_{1}=\mathrm{H}$

$440 \mathrm{R}=\mathrm{CHCH}_{2} \mathrm{OH}, \mathrm{R}_{1}=\mathrm{OCH}_{3}$

$441 \mathrm{R}=\mathrm{CHCHO}, \mathrm{R}_{1}=\mathrm{H}$

$442 \mathrm{R}=\mathrm{O}, \mathrm{R}_{1}=\mathrm{H}$<smiles>COc1cc(C(O)C(O)CO)cc2c1OC(c1ccc(O)cc1)C2CO</smiles><smiles>CNC(=O)/C=C/c1cc(OC(C)=O)c2c(c1)[C@H](CO)[C@H](c1ccc(O)c(OC)c1)O2</smiles>

433<smiles>CNC(=O)/C=C/c1cc(OC)c2c(c1)C(C(=O)N[Tl])[C@H](c1ccc(O)c(OC)c1)O2</smiles><smiles>COc1cc(C[C@@H]2CO[C@H](c3ccc(OC4CCCCC4)c(OC)c3)[C@H]2CO)ccc1OC1CCCCC1</smiles>

Fig. 9 Lignans 414-444 from Solanum 
<smiles>COc1cc(/C=C/C(=O)N[C@H]2CCCN2C(=N)N)ccc1/C=C/C(=O)NC1CCCN1C(=N)N</smiles><smiles>CCOC(=O)c1cc(=O)[nH]c(=O)[nH]1</smiles><smiles>CO[C@H](CNC(=O)/C=C/c1ccc(O)cc1)c1ccc(O)cc1</smiles>

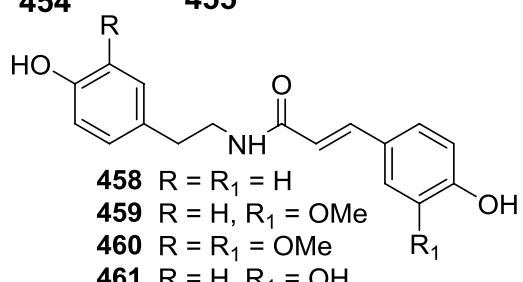

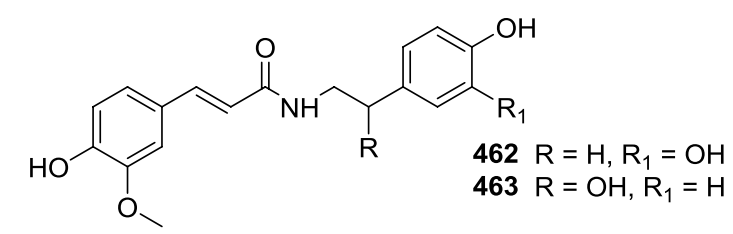

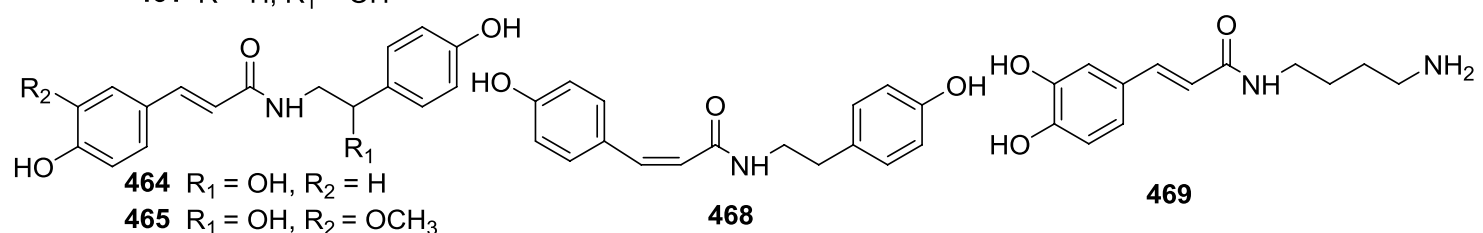
$\mathrm{R}_{1}=\mathrm{OH}, \mathrm{R}_{2}=\mathrm{OCH}_{3}$ $466 R_{1}=H, R_{2}=H$ $467 \mathrm{R}_{1}=\mathrm{H}, \mathrm{R}_{2}=\mathrm{OCH}_{3}$

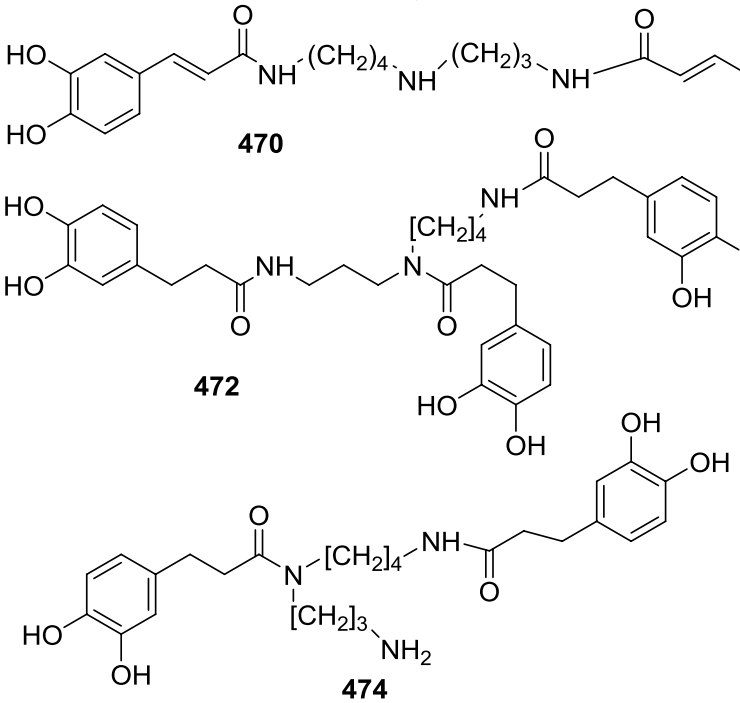<smiles>CC(=O)OC[C@H](Cc1ccccc1)NC(=O)[C@H](Cc1ccccc1)NC(=O)c1ccccc1</smiles>

471<smiles>CN(CCCNC(=O)CCc1ccc(O)c(O)c1)C(=O)CCc1ccc(O)c(O)c1</smiles>

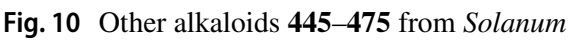

\subsection{Phenolic Compounds}

Fifty-two phenolic compounds (542-593) were recorded from Solanum (Fig. 12). The fruits of S. crinitum have yielded 552, 561-564 [122]. Aerial parts of $S$. torvum indicated a great wealth of phenolic compunds, e.g. 558-559,
576, 591-593 [315, 320, 335-337, 521, 524, 533]. The highest numbers of phenols, 542-546, 549-540, 552, 555 and $\mathbf{5 8 9}$ were reported from stems of S. melongena [205] while 574-575 and 577-584 were mentioned from the fruits $S$. sessiliflorum [525]. 


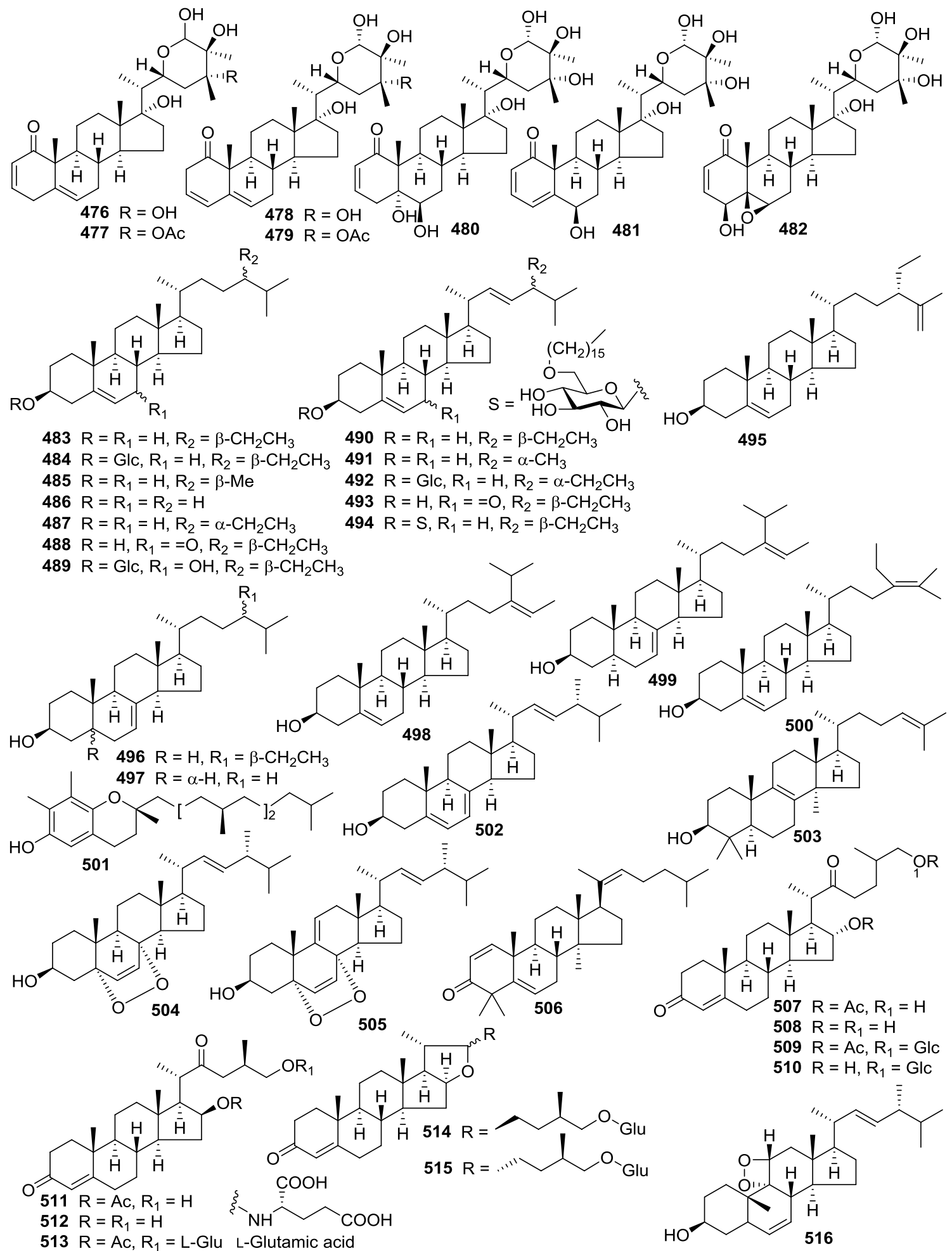

Fig. 11 Sterols 476-541 from Solanum

Phenolic compounds in Solanum have displayed antibacterial [297, 320, 335-337, 524], anticancer [31], antidiabetic [297, 320, 335-337, 524] and antihypertensive [521] activities. Chlorogenic acid (546) (21.90 \pm 0.02 mgg), gallic acid (551) (17.54 $\pm 0.04 \mathrm{mgg})$ and caffeic acid (555) (16.64 $\pm 0.01 \mathrm{mgg})$ have indicated potent and concentration-dependent DPPH radical-scavenging activity $\left(\mathrm{IC}_{50}=275.03 \pm 7.8 \mu \mathrm{g} / \mathrm{mL}\right)[31]$, and $\mathbf{5 5 1}$ and $\mathbf{5 5 5}$ 


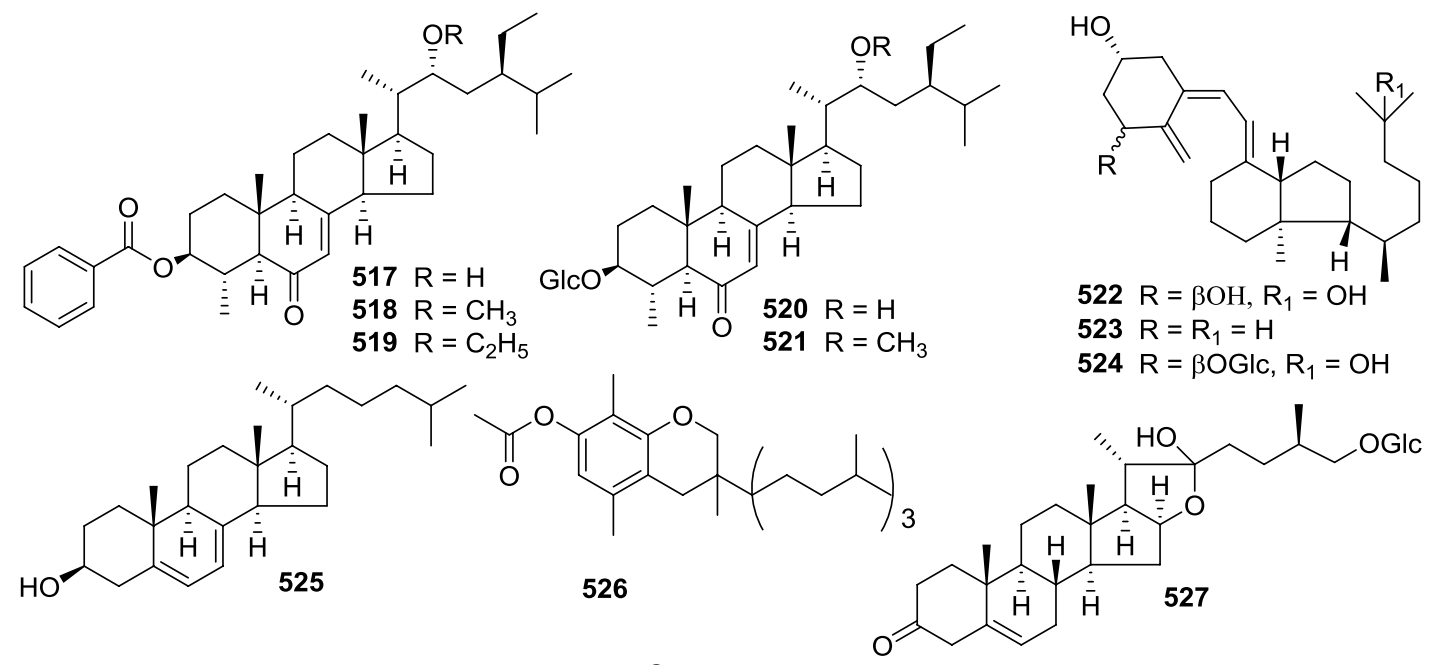

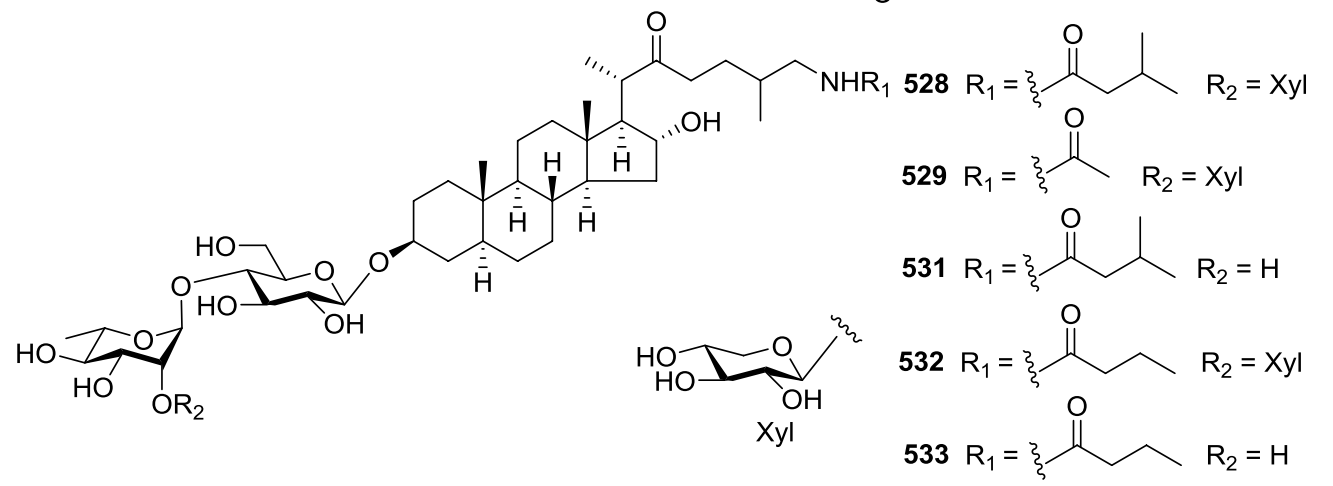

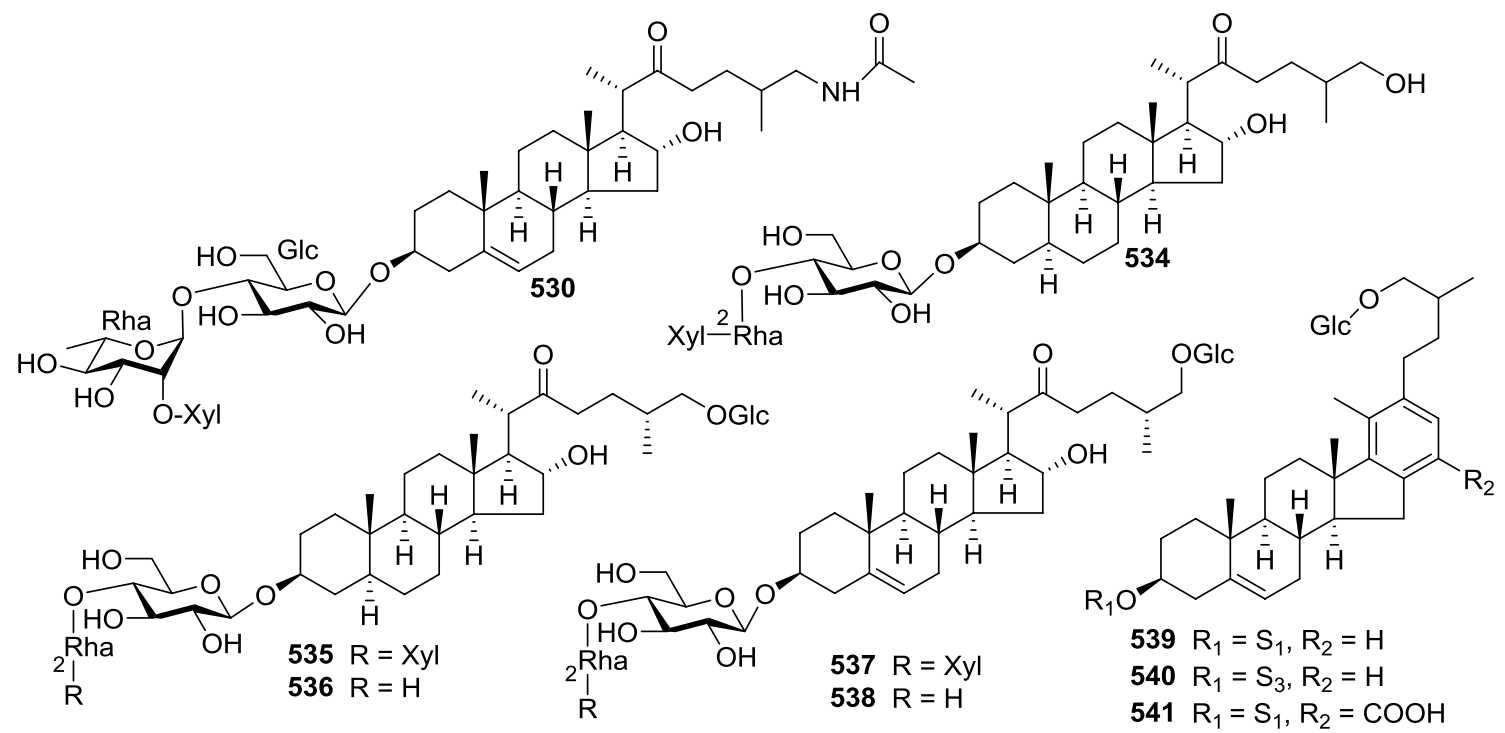

Fig. 11 (continued)

reportedly have great potentials as natural source of antidiabetic and antioxidant drug [336]. trans-Cinnamic acid (590) showed antibacterial activities ( $\mathrm{MIC}=250 \mu \mathrm{g} / \mathrm{mL}$ ) against Staphylococcus aureus [297], and antimycobacterial activities (inhibition zone $=0-22 \mathrm{~mm}$ ) against Proteus vulgaris, Klebsiella pneumoniae (ESBL-), M. tuberculosis
$\left(\mathrm{H}^{37} \mathrm{Rv}\right)$ and M. tuberculosis (Rifampin) [320]. Methyl caffeate (591) not only significantly reduced the cell proliferation, but also increased formation of fragmented DNA and apoptotic body in MCF-7 cells. In this study, Bcl-2, Bax, Bid, p53, caspase-3, PARP and cytochrome c release were detected by western blot analyses [474]. 
<smiles>[R]C1[C@H](O)[C@@H](O)[C@](O)(C(=O)O)C[C@H]1O</smiles>

Caffeoyl<smiles>[R]C1CC(O)(C(=O)O)CC([R])C1O</smiles>

$543 \mathrm{R}=$ Caffeoyl, $\mathrm{R}_{1}=\mathrm{OH}$

$544 \mathrm{R}=$ Caffeoyl, $\mathrm{R}_{1}=\mathrm{OAc}$ $549 \mathrm{R}=\mathrm{R}_{1}=$ Caffeoyl

$540 \mathrm{R}=(Z)$ Caffeoyl, $\mathrm{R}_{1}=\mathrm{OH}$<smiles>[R]C1[C@H](O)C[C@@](O)(C(=O)O)C[C@H]1OC(C)=O</smiles>

545<smiles>[R]C1CC([R1])(C(=O)O)CC([O])C1[R2]</smiles>

$546 \mathrm{R}=$ Caffeoyl, $\mathrm{R}_{1}=\mathrm{R}_{2}=\alpha-\mathrm{OH}$

$547 \mathrm{R}=$ Caffeoyl, $\mathrm{R}_{1}=\mathrm{R}_{2}=\beta-\mathrm{OH}$<smiles>[R]C(Cc1ccc(O)c(O)c1)C(=O)O</smiles><smiles>[R]CC=CC(=O)O</smiles><smiles>[R]c1ccc(/C=C/C(=O)O)cc1[R]</smiles>
$555 \mathrm{R}=\mathrm{R}_{1}=\mathrm{OH}$ $556 \mathrm{R}=\mathrm{H}, \mathrm{R}_{1}=\mathrm{OH}$ $557 \mathrm{R}=\mathrm{OH}, \mathrm{R}_{1}=\mathrm{OMe}$<smiles>COc1cc(OC)c(O)c(OC)c1</smiles>
$R_{1} 552 R=R_{1}=H$ $553 \mathrm{R}=\mathrm{OH}, \mathrm{R}_{1}=\mathrm{H}$ $554 \mathrm{R}=\mathrm{OMe}, \mathrm{R}_{1}=\mathrm{H}$<smiles>CCCCCCCCCCCCCC=Cc1ccc(O)cc1</smiles><smiles>[R]OC(=O)/C=C/c1ccc(O)cc1</smiles>
$561 \mathrm{R}=\mathrm{CH}_{2} \mathrm{CH}_{3}$ $562 \mathrm{R}=\mathrm{H}$<smiles>[R]OC(=O)/C=C/c1ccc(O)cc1</smiles><smiles>[R][R]([H])([Sb])P([R])(=O)O</smiles><smiles>CCOC(=O)/C=C/c1ccc(O)c(O)c1</smiles>

$\mathrm{R}_{1} \mathrm{O}$<smiles>[R][R]([R])=O</smiles><smiles>[R]Oc1cc(C=O)ccc1O</smiles>
$571 \mathrm{R}=\mathrm{Me}$ $572 \mathrm{R}=\mathrm{H}$<smiles>[R16]C[C@]1(O)C[C@@H](O)[C@H](O)[C@H](O)C1</smiles><smiles>[R]c1ccccc1[R]</smiles>
$569 \mathrm{H} \quad \mathrm{OH} \quad \mathrm{Me}$

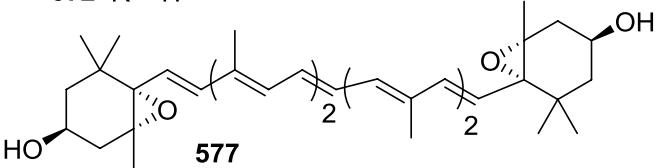

$576 \mathrm{R}=\mathrm{OH}, \mathrm{R}_{1}=\mathrm{COOH}$<smiles>CC1=C[C@H](O)CC(C)(C)[C@H]1/C=C(C)/C(C)=C/C=C(C)/C=C(C)/C=C(C)/C=C(\C)C1=C(C)C[C@@H](O)CC1(C)C</smiles><smiles>CC(C)=C/C(C)=C/C(C)=C/C(C)=C/C(C)=C/C=C(C)/C(C)=C/C1C(C)=CCCC1(C)C</smiles>

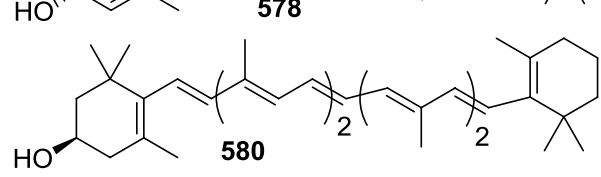<smiles>CC(C=CC12OC1(C)CC(O)CC2(C)C)=CC=CC(C)(C)CC=CC=C(C)C1C=C2C(C)(C)CC(O)CC2(C)O1</smiles><smiles>CCCCC(C)=CC(C)=CC(C)=CC(C)=CC(C)=CC(C)=CC1=C(C)CCCC1(C)C</smiles><smiles>C=C(C)/C=C/C=C(C)/C=C/C1=C(C)C(C)(C)C[C@H](O)C[C@@]1(C)O</smiles>

584

585<smiles>C/C=C(C)/C=C/C=C(C)\C=C\C12OC1(C)C[C@@H](O)CC2(C)C</smiles><smiles>CC(C=CC1C(C)=CCCC1(C)C)=CC(C)=CC(C)=CC(C)=CC(C)=CC1=C(C)C[C@@H](O)CC1(C)C</smiles><smiles>COc1cc(C(O)C(CO)c2ccc(O)c(OC)c2)ccc1O</smiles><smiles>C=CCc1cc(OC)c(O)cc1S(=O)(=O)c1ccccc1</smiles><smiles>[R]C=Cc1ccc([R])c([R])c1</smiles>

$589 \mathrm{R}=\mathrm{OMe}, \mathrm{R}_{1}=\mathrm{OH}, \mathrm{R}_{2}=\mathrm{CHO}$<smiles>O=C(/C=C/c1ccc(O)c(O)c1)O[C@H]1CC[C@@H](O)[C@@H]1O</smiles>

$590 \mathrm{R}=\mathrm{R}_{1}=\mathrm{H}, \mathrm{R}_{2}=\mathrm{COOH}$

$591 \mathrm{R}=\mathrm{R}_{1}=\mathrm{OH}, \mathrm{R}_{2}=\mathrm{COOCH}_{3}$

Fig. 12 Phenolic compounds 542-593 from Solanum 


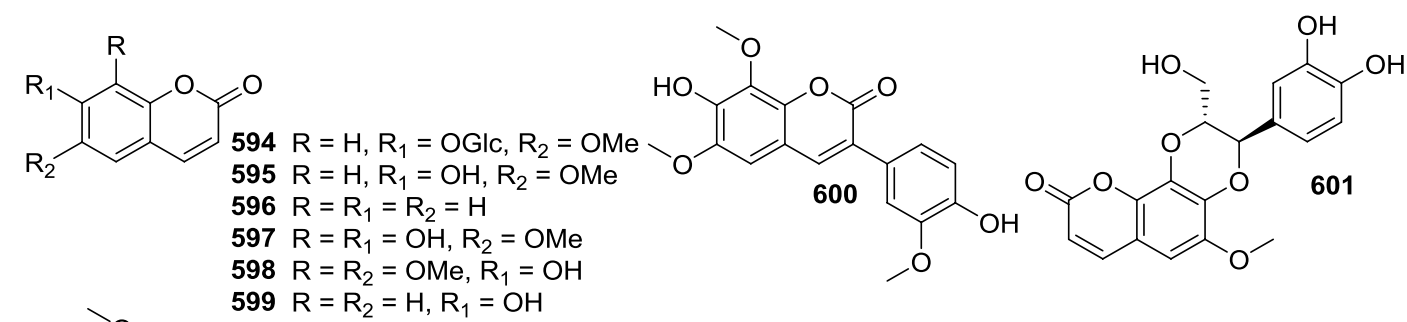<smiles>COc1cc2c(-c3cc(=O)oc4c(OC)c(O)c(OC)cc34)cc(=O)oc2c(OC)c1O</smiles><smiles>COc1ccc(OC)c(O)c1</smiles>

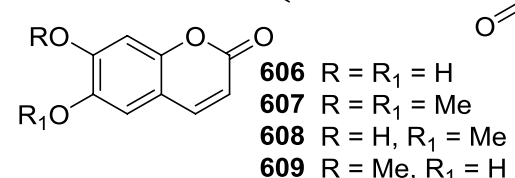<smiles>CCC(C=CC(=O)O)C(=O)O</smiles><smiles>COc1cc(/C=C2/Oc3c(c(C)cc4ccc(=O)oc34)OC2=O)ccc1O</smiles>

604<smiles>O=c1oc2cc(O)ccc2c2oc3cc(O)ccc3c12</smiles><smiles>CC1(C)C=Cc2cc3c(cc2O1)oc(=O)c1c2cc(O)ccc2[nH]c31</smiles><smiles></smiles>

Fig. 13 Coumarins and coumestans 594-613 from Solanum<smiles>COc1cc([C@H]2Oc3c(OC)cc4c(c3OC(=O)C=C4)O[C@@H]2C(=O)NCCc2ccc(O)cc2)ccc1O</smiles>

614<smiles>COc1cc([C@H]2Oc3c(OC)cc4c(c3OC(=O)C=C4)O[C@@H](C(=O)NCC(=O)c3c[nH]c4ccccc34)[C@H]2O)ccc1O</smiles>

615<smiles>[R]OC(=O)[C@H]1Oc2c(OC)cc3ccc(=O)oc3c2O[C@H]1c1ccc(O)c(OC)c1</smiles>

Fig. 14 Coumarinolignoids 614-617 from Solanum

The effects of oral administration of $\mathbf{5 9 1}(10,20$ and 40 $\mathrm{mgkg}$ ) in streptozotocin induced diabetic rats, including body weight, fasting blood glucose, plasma insulin, hemoglobin, glycated hemoglobin, total protein, hepatic glycogen and carbohydrate metabolism enzymes have been studied for 28 days. At $40 \mathrm{mgkg}$, the compound significantly prevented the increase in blood glucose level after glucose administration at $60 \mathrm{~min}$ in comparison to the hyperglycemic control group. It also produced remarkable reductions in blood glucose and increased body weight in streptozotocin induced diabetic rats [335]. Takahashi et al. further established that $\mathbf{5 9 1}$ has a most favorable structure for both sucrase and maltase inhibition against sucrose and that its moderate inhibitory action against alpha-glucosidase provides a prospect for antidiabetic usage of $S$. torvum fruit [337].

\subsection{Coumarins and Coumestans}

Seventeen coumarins 594-610 and three coumastans 611-613 were isolated from Solanum spp. (Fig. 13). The seeds of $S$. indicum yielded the highest number of 


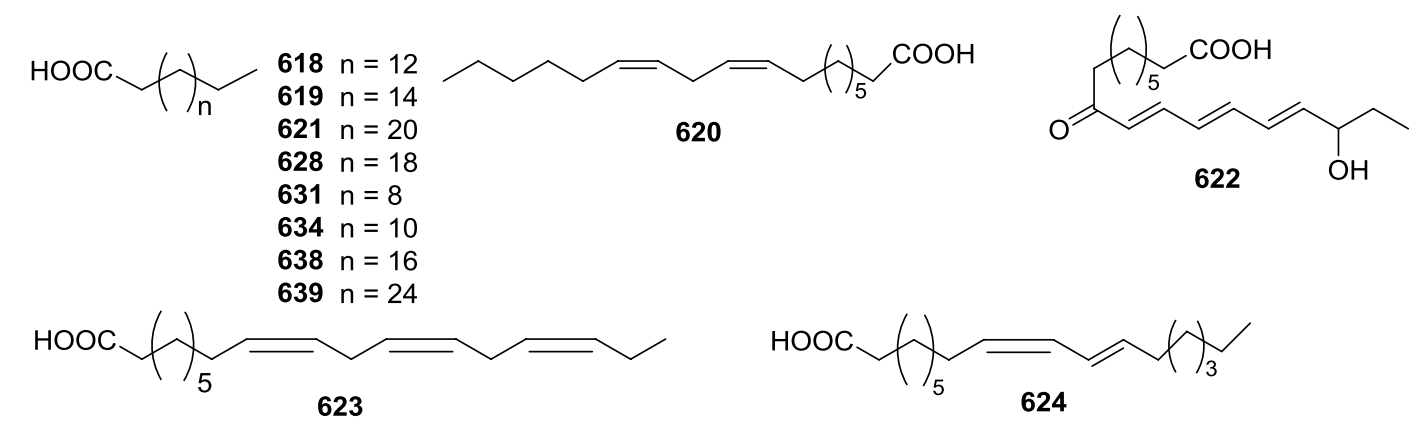<smiles>CCCCCC(O)/C=C/C=C/C1CC2CC1C2</smiles>

625<smiles>CC/C=C/C/C=C/C=C/C(O)CC12CCC(CC1)C2</smiles><smiles>CCC(C)(C)CCC(C)(C)C(=O)O</smiles>

627<smiles>CCC(C)(C)/C=C\CC(C)(C)C(C)(C)C(=O)O</smiles><smiles>CC(C)(C)/C=C/C(C)(CCCC(=O)O)CCCC(=O)O</smiles>

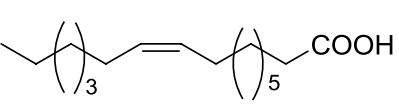

632<smiles>CCC(C)CC(C)(C)CCCC(=O)O</smiles><smiles>CCC=CCC=CCC=CCCCCCC(=O)O</smiles><smiles>CC(C)=CC=CC(=O)CC1CC1CC(=O)O</smiles><smiles>CC(C)(C)C=CC=CC(=O)CCCC(=O)O</smiles><smiles>C=CC(=O)OCCCCc1cc(C(C)(C)C)c(O)c(C(C)(C)C)c1</smiles>

Fig. 15 Fatty acids and esters 618-640 from Solanum

coumarins 597-598 and 600-604 [535, 536], while coumestans 611-613 were from the whole plant of $S$. lyratum [88]. Scopolin (594), scopoletin (595) and coumarin (596) are the main coumarins in Solanum. Compounds 611-613 showed in vitro anti-inflammatory activities with $\mathrm{IC}_{50}$ values in the range of 6.3-9.1 $\mu \mathrm{M}$ [88].

\subsection{Coumarinolignoids}

Four coumarinolignoids known as indicumines A-D (614-617) were obtained from the seeds of $S$. indicum [535] (Fig. 14). Coumarinolignoids, including cleomiscosins, aquillochins and malloapelins, are unique and rare in nature. Coumarinolignoids of the cleomiscosins type bearing cleomiscosins A-D, 8-epi-cleomiscosin A, and malloapeli A functionalities have been identified in a few genera, including Cleome viscosa, Mallotus apelta, and Rhododendron collettianum. The compounds with such functionalities, especially cleomiscosins $\mathrm{A}-\mathrm{C}$ and 8-epicleomiscosin A, which contributed to biological activities, have been reported with hepatoprotective and tyrosinase inhibition activities [535].

\subsection{Fatty Acids and Esters}

Nine saturated $(618-619,621,627-628,631,634,638-639)$ and 13 unsaturated $(\mathbf{6 2 0}, \mathbf{6 2 2}-\mathbf{6 2 6}, \mathbf{6 2 9}, \mathbf{6 3 0}, \mathbf{6 3 2}, \mathbf{6 3 3}$, 635-637, 640) fatty acids were reported from Solanum (Fig. 15). The whole plant of S. glabratum has yielded the highest number of fatty acid and esters (627-635) in Solanum spp. [140]. Hexadecanoic acid (618), notably the major fatty acid component in Solanum, was isolated from aerial parts of S. aculeastrum [11] S. vestissimum [489] and S. villosum $[434,479]$.

\subsection{Others}

Thirty other kinds of compounds (641-670) were also obtained from Solanum spp. (Fig. 16). Most of them, 642-653, were from the leaves of $S$. aculeastrum [11] and 654-659 were yielded from the fruits of $S$. betaceum [78]. An aldehyde puerariafuran (641) and a cyclic eight-membered $\alpha, \beta$-unsataturated ketone, solalyratin $\mathrm{B}(\mathbf{6 6 1})$ were isolated from the whole plant of $S$. lyratum [88]. Compounds $\mathbf{6 4 1}$ and 661 showed in vitro anti-inflammatory activities, with $\mathrm{IC}_{50}$ values in the range 6.3-9.1 $\mu \mathrm{M}$ [88]. Also presented here are two furans, ethyl- $\alpha$-D-arabinofuranoside 


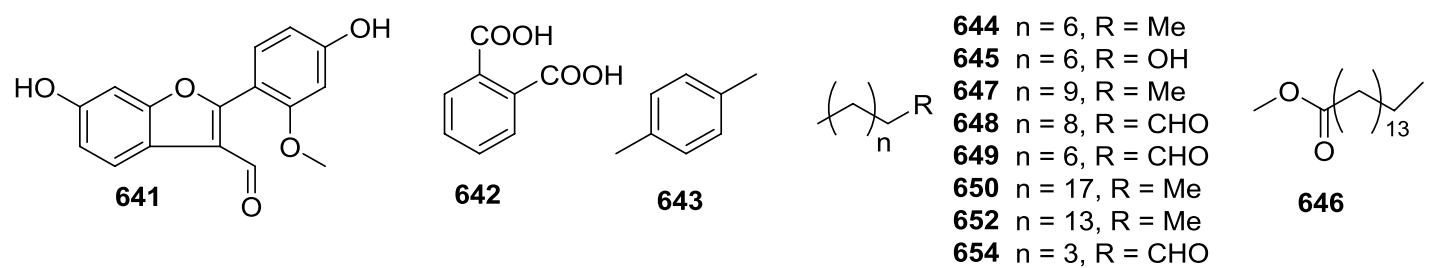

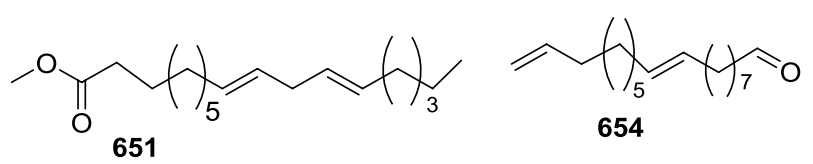<smiles>CCOC(=O)CCCC(C)(C)O</smiles>

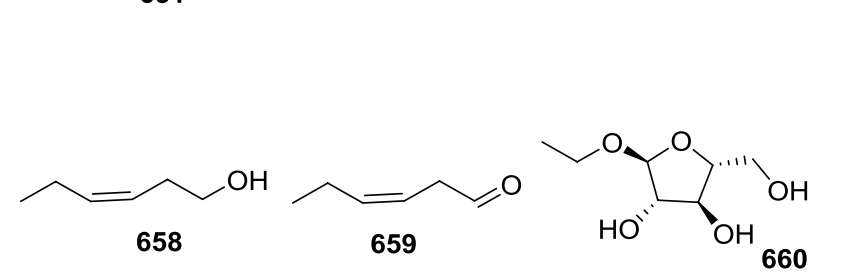<smiles>CC(=O)/C=C/[C@]1(O)C(C)=CC(=O)CC(C)(C)[C@@]1(C)O/C1=C/C(=O)C(C)(C)C[C@H](O)C[C@H]1C</smiles><smiles>CC(O)COC(C)COC(C)COC(C)O</smiles><smiles>COc1cc(/C=C/CO)cc2c(CO)coc12</smiles>

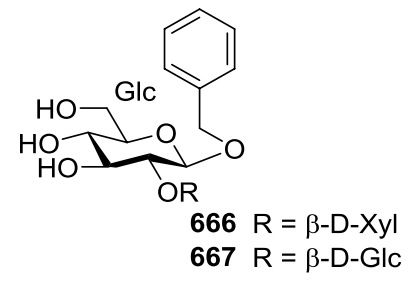

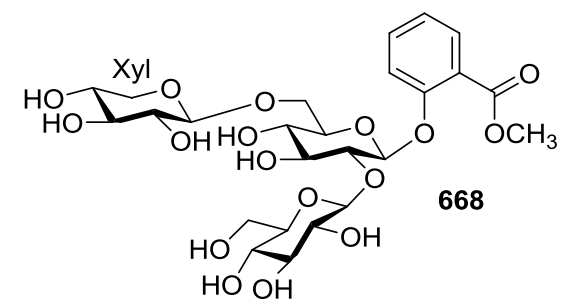

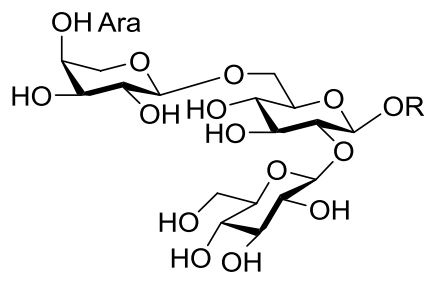<smiles>CCCc1ccc(CN)cc1</smiles>

Fig. 16 Other compounds 641-670 from Solanum

(660) from the whole plant of S. lyratum and 5-hydroxymethyl furfural (663) from the stems of $S$. torvum [533]. Five aromatic glycosides (666-670) were also isolated from the aerial part of S. incanum [494] and the fruit of S. lycopersicum [511].

\section{Conclusion and Future Prospects}

From 1990 to 2017, phytochemical studies on the 65 Solanum species have yielded at least 670 compounds (134 steroidal saponins, 63 steroidal alkaloids, 13 pregnane glycosides, 128 terpenes, 75 flavonoids, 31 lignans, 31 alkaloids, 66 steroids, 52 phenolic compounds, 20 coumarins and coumestans, 4 coumarinolignoids, 23 fatty acids and esters, and 30 other types of compounds).

Pharmacological studies on Solanum genus have focused on antioxidants and anticancer activities. A total of 17 species (fruits of S. aculeastrum, S. americanum,
S. muricatum, S. sessiliflorum and S. spirale, seeds of $S$. capsicoides, the stems of $S$. cathayanum and $S$. tuberosum, the roots of $S$. diphyllum, aerial parts of $S$. surattense and $S$. torvum and the whole plant parts of $S$. aethiopicum, $S$. nigrum, S. anguivi, S. septemlobum, $S$. violaceum and $S$. xanthocarpum) have been explored for anticancer activities and have exhibited significant results.

S. xanthocarpum has outstandingly demonstrated the most diverse pharmacological activities $e . g$. antioxidants and antitumor, anti-fungal, anti-bacterial, antileishmanial, mosquito larvicidal, molluscicidal, antidiabetic, asthmatic, hepatoprotective, diuretic, nephrotoxicity, antinociceptive, anti-psoriatic, and antiurolithiatic.

Steroidal alkaloids have been presented as being largely responsible for various pharmacological activities of Solanum species, e.g. antibacterial $(\mathbf{1 3 9}, 141$ and 145), anticonvulsant and CNS depressant (145), antidiabetic (139, 142 and 144), anti-fungal (145 and 174), anti-inflammatory (145), antileishmanial (139 and 142), molluscicidal (139 
and 141), nephrotoxicity (168), antioxidants and antitumor $(139,141,145,158,168$ and 180), antiprotozoa (139 and 142), schistosomicidal (139 and 142), spasmolytic (190) and anti-trypanosomal (139).

The genus Solanum seems to possess great potential, yet majority of the species remain unknown or scantily studied for the chemical constituents. It would be very necessary for the phytochemistry researchers to explore and investigate more of its species. The vast pharmacological activities envinced by many compounds from Solanum genus should attract the attention of the pharmacological community to determine their exact target sites, structure-activity relationships and other medicinal applications.

\section{Compliance with Ethical Standards}

Conflict of interest The authors declare no conflict of interest.

Open Access This article is distributed under the terms of the Creative Commons Attribution 4.0 International License (http://creativeco mmons.org/licenses/by/4.0/), which permits unrestricted use, distribution, and reproduction in any medium, provided you give appropriate credit to the original author(s) and the source, provide a link to the Creative Commons license, and indicate if changes were made.

\section{References}

1. https://en.wikipedia.org/wiki/Solanum. Accessed 22 Aug 2017

2. https://en.wikipedia.org/wiki/Solanum_abutiloides. Accessed 22 Aug 2017

3. T. Yokose, K. Katamoto, S. Park, H. Matsuura, T. Yoshihara, Biosci. Biotechnol. Biochem. 68, 2640-2642 (2004)

4. H. Yoshimitsu, M. Nishida, T. Nohara, Phytochemistry 64, 13611366 (2003)

5. H. Yoshimitsu, M. Nishida, M. Yoshida, T. Nohara, Chem. Pharm. Bull. 50, 284-286 (2002)

6. H. Yoshimitsu, M. Nishida, T. Nohara, Chem. Pharm. Bull. 48, $556-558$ (2000)

7. R.H. Tian, E. Ohmura, M. Matsui, T. Nohara, Phytochemistry 44, 723-726 (1997)

8. R.H. Tian, E. Ohmura, H. Yoshimitsu, T. Nohara, M. Matsui, Chem. Pharm. Bull. 44, 1119-1121 (1996)

9. E. Ohmura, T. Nakamura, R.H. Tian, S. Yahara, H. Yoshimitsu, T. Nohara, Tetrahedron Lett. 36, 8443-8444 (1995)

10. http://pza.sanbi.org/solanum-aculeastrum. Accessed 22 Aug 2017

11. S. Koduru, O.T. Asekun, D.S. Grierson, A.J. Afolayan, J. Ess, Oil-Bear. Plants 9, 65-69 (2006)

12. A.W. Wanyonyi, S.C. Chhabra, G. Mkoji, W. Njue, P.K. Tarus, Fitoterapia 74, 298-301 (2003)

13. S. Koduru, F.O. Jimoh, D.S. Grierson, A.J. Afolayan, J. Pharm. Toxicol. 2, 160-167 (2007)

14. S. Koduru, D.S. Grierson, M. Van de Venter, A.J. Afolayan, Pharm. Biol. 45, 613-618 (2007)

15. T. Burger, V. Steenkamp, W. Cordier, T. Mokoka, G. Fouche, P. Steenkamp, BMC Comp. Alt. Med. 18, 137 (2018)

16. L.M. Njoki, S.A. Okoth, P.M. Wachira, Int. J. Microbiol. 2017, 5273893 (2017)
17. K.K. Francisca, M. Jacob, L.K. Omosa, J. Nganga, N. Maina, J. Ethnopharmacol. 192, 524-534 (2016)

18. L.T. Laban, J.M. Mutiso, S.G. Kiige, M.M. Ngedzo, Iran. J. Basic. Med. Sci. 18, 64-71 (2015)

19. A.W. Wanyonyi, S.C. Chhabra, G. Mkoji, U. Eilert, W.M. Njue, Phytochemistry 59, 79-84 (2002)

20. https://en.wikipedia.org/wiki/Solanum_aethiopicum. Accessed 22 Aug 2017

21. http://www.pfaf.org/user/Plant.aspx ?Latin Name=Solan um+aethiopicum. Accessed 22 Aug 2017

22. A. Chioma, A. Obiora, U. Chukwuemeka, J. Trop. Med. 4, 163-166 (2011)

23. K.C. Kouassi, B.J.A. Mamyrbekova, Res. J. Rec. Sci. 4, 81-87 (2015)

24. C.A. Anosike, N.E. Ogbodo, A.L. Ezugwu, R.I. Uroko, C.C. Ani, O. Abonyi, Am.-Eur J. Toxicol. Sci. 7, 104-109 (2015)

25. A. Adetutu, O.S. Olorunnisola, E.B. Oyewo, Can. J. Pure Appl. Sci. 7, 2357-2362 (2013)

26. E.E. Nwanna, E.O. Ibukun, G. Oboh, Adv. Food Sci. 35, 30-36 (2013)

27. C.A. Anosike, O. Obidoa, L.U.S. Ezeanyika, Asian Pac. J. Trop. Med. 5, 62-66 (2012)

28. C. Tagawa, M. Okawa, T. Ikeda, T. Yoshida, T. Nohara, Tetrahedron Lett. 44, 4839-4841 (2003)

29. T. Nagaoka, K. Goto, A. Watanabe, Y. Sakata, T. Yoshihara, J. Biosci. 56, 707-713 (2001)

30. https://en.wikipedia.org/wiki/Solanum_anguivi. Accessed 22 Aug 2017

31. O.O. Elekofehinti, J.P. Kamdem, A.A. Bolingon, M.L. Athayde, S.R. Lopes, Asian Pac. J. Trop. Biomed. 3, 757-766 (2013)

32. C. Dalavi, S. Patil, Int. J. Pharm. Sci. Rev. Res. 30, 112-120 (2016)

33. O.O. Elekofehinti, J.P. Kamdem, I.J. Kade, J.B.T. Rocha, S. Afr. J. Bot. 88, 56-61 (2013)

34. O.O. Elekofehinti, I.G. Adanlawo, J.A. Saliu, S.A. Sodehinde, Pharm. Lett. 4, 811-814 (2012)

35. O.O. Elekofehinti, I.G. Adanlawo, A. Fakoya, Asian J. Pharm. Health Sci. 2, 416-419 (2012)

36. O.O. Elekofehinti, J.P. Kamdem, D.F. Meinerz, I.J. Kade, I. Joseph, Arch. Pharmacal Res. (2015)

37. O.O. Elekofehinti, J.P. Kamdem, I.J. Kade, I.G. Adanlawo, Asian J. Pharm. Clin. Res. 6, 252-257 (2013)

38. J. Gandhiappan, R. Rengasamy, Pharm. Lett. 4, 875-880 (2012)

39. O.O. Elekofehinti, I.G. Adanlawo, J.A. Saliu, S.A. Sodehinde, Curr. Res. J. Biol. Sci. 4, 530-533 (2012)

40. I.G. Adanlawo, M.A. Akanji, Rec. Prog. Med. Plants 19, 1-7 (2008)

41. X.H. Zhu, T. Ikeda, T. Nohara, Chem. Pharm. Bull. 48, 568-570 (2000)

42. H. Ripperger, U. Himmelreich, Phytochemistry 37, 1725-1727 (1994)

43. T. Honbu, T. Ikeda, X.H. Zhu, O. Yoshihara, M. Okawa, A.M. Nafady, T. Nohara, J. Nat. Prod. 65, 1918-1920 (2002)

44. http://eol.org/pages/5695130/data. Accessed 22 Aug 2017

45. A.C. Correia, C.L. Macedo, F.S. Monteiro, G. Alves de Oliveira, J. Med. Plants Res. 7, 2293-2299 (2013)

46. http://www.fireflyforest.com/flowers/2364/solanum-americanum -american-black-nightshade/. Accessed 22 Aug 2017

47. https://en.wikipedia.org/wiki/Solanum_americanum. Accessed 22 Aug 2017

48. J.N. Kadima, F.M. Kasali, B. Bavhure, A.O. Mahano, F.M. Bwironde, Int. J. Pharmacol. Pharm. 5, 196-206 (2016)

49. E.L. Silva, L.R.C. Almeida, R.M. Borges, D. Staerk, Fitoterapia 118, 42-48 (2017) 
50. J.M. Vagula, J. Bertozzi, J.C. Castro, O.C. Celestino, Nat. Prod. Res. 30, 2230-2234 (2016)

51. I. Fidrianny, A. Rizkiya, K. Ruslan, J. Chem. Pharm. Res. 7, 666-672 (2015)

52. L.U. Colmenares, J. Lai, Determination of Phenolic Content, Antioxidant Activity and Mineral Content of Popolo (Solanum americanum) Leaves. Abstracts, 64th Northwest Regional Meeting of the American Chemical Society, (Tacoma, WA, United States, 2009)

53. B.E. Hope, D.G. Massey, M.G. Fournier, Hawaii Med. J. 52, 160-166 (1993)

54. V.A. Maritza, N.L. Clara, Afinidad 56, 393-396 (1999)

55. L. Al Chami, R. Mendez, B. Chataing, J. O'Callaghan, A. Usubillaga, Phytother. Res. 56, 254-258 (2003)

56. A. Vazquez, F. Ferreira, P. Moyna, L. Kenne, Phytochem. Anal. 10, 194-197 (1999)

57. F. Ferreira, A. Vazquez, P. Moyna, L. Kenne, Phytochemistry 36, 1473-1478 (1994)

58. http://www.boldsystems.org/index.php/Taxbrowser_Taxon page?taxid=148709. Accessed 22 Aug 2017

59. A. Maxwell, R. Pingal, W.F. Reynolds, S. McLean, Phytochemistry 43, 913-915 (1996)

60. F. Londono, W. Cardona, F. Alzate, F. Cardona, I.D. Velez, J. Med. Plants Res. 10, 100-107 (2016)

61. C.M. Chinchilla, C.I. Valerio, P.R. Sanchez, M.V. Bagnarello, C.D. Rodriguez, Rev. Biol. Trop. 62, 1229-1240 (2014)

62. M. Chinchilla, I. Valerio, R. Sanchez, V. Mora, V. Bagnarello, L. Martinez, Rev. Biol. Trop. 60, 881-891 (2012)

63. A. Maxwell, R. Pingal, W.F. Reynolds, M.S. McLean, Stewart Phytochem. 42, 543-545 (1996)

64. K. Fukuhara, K. Shimizu, I. Kubo, Arudonine Phytochem. 65, 1283-1286 (2004)

65. M.H. Grace, M.M. Saleh, Pharm 51, 593-595 (1996)

66. T.M.S. Silva, C.A. Camara, K.R.L. Freire, T.G. da Silva, J. Braz. Chem. Soc. 19, 1048-1052 (2008)

67. F.C.L. Pinto, D.E.A. Uchoa, E.R. Silveira, O.D.L. Pessoa, L.S. Espindola, Quim. Nova 34, 284-288 (2011)

68. P.C.B. Silva, N.J. Clementino, A.D.S. da Silva, S. da Melo, S. Karoline, M.S. Tania, Rev. Bras. Farmacogn. 22, 131-136 (2012)

69. R.C.M. Oliveira, J.T. Lima, L.A.A. Ribeiro, J.L.V. Silva, F.S. Monteiro, J. Biosci. 61, 799-805 (2006)

70. T.M.S. Silva, R.A. Costa, E.J. Oliveira, F.J.M. Barbosa, J. Braz. Chem. Soc. 16, 1467-1471 (2005)

71. http://www.hear.org/pier/species/solanum_betaceum.htm. Accessed 23 Aug 2017

72. https://en.wikipedia.org/wiki/Tamarillo. Accessed 23 Aug 2017

73. http://eol.org/pages/486416/overview. Accessed 23 August 2017

74. http://www.iucnredlist.org/details/34636/0. Accessed 23 Aug 2017

75. N.H. Hurtado, A.L. Morales, M.L. Gonzalez-Miret, M.L. Escudero-Gilete, F. Heredia, Food Chem. 117, 88-93 (2009)

76. C. Osorio, N. Hurtado, C. Dawid, T. Hofmann, M. Heredia, J. Francisco, A.L. Morales, Food Chem. 132, 1915-1921 (2012)

77. A.A. Durant, C. Rodriguez, A.I. Santana, C. Herrero, J.C. Rodriguez, Rec. Nat. Prod. 7, 15-26 (2013)

78. G.J. Maria, P.L. Juliana, O. Coralia, G. Alirio, M. Diana, Mol. 21, (2016)

79. F.D.C.L. Pinto, M.D.C.M. Torres, E.R. Silveira, Quim. Nova 36, 1111-1115 (2013)

80. N.P. Vaz, E.V. Costa, E.L. Santos, S.B. Mikich, F.A. Marques, R.A. Braga, J. Braz. Chem. Soc. 23, 361-366 (2012)

81. https://en.wikipedia.org/wiki/Solanum_capsicoides. Accessed 24 Aug 2017

82. http://florida.plantatlas.usf.edu/Plant.aspx?id=609. Accessed 24 Aug 2017
83. http://www.flora.sa.gov.au/efsa/lucid/Solanaceae/Solanum $\% 20$ species/key/Australian\%20Solanum\%20species/Media/Html/ Solanum_capsicoides.htm. Accessed 24 Aug 2017

84. http://davesgarden.com/guides/pf/go/38381/. Accessed 24 Aug 2017

85. B.W. Chen, Y.Y. Chen, Y.C. Lin, RSC Adv. 5, 88841-88847 (2015)

86. M. Petreanu, A.A.A. Guimaraes, M.F. Broering, Nau.-Sch Arch Pharm. 389, 1123-1131 (2016)

87. L.O. Simoes, F.G. Conceicao, T.S. Ribeiro, A.M. Jesus, D.F. Silva, Phytomedicine 23, 498-508 (2016)

88. J. He, B.Z. Ma, X.F. Tian, F.L. Wei, T. Zhao, Chin. Pharm. Mag. 25, 3713-3718 (2014)

89. S. Xu, L. Wang, H. Liu, Shizhen Chin. Med. J. 17, 523-524 (2006)

90. G.M. Nino, O.V. Urias, M.D.R. Muy, J.B. Heredia, S. Afr. J. Bot 111, 161-169 (2017)

91. J. Sun, Y.F. Gu, M.M. Li, X.Q. Su, H. Li, J. Li, P.F. Tu, Chin. Herb. Med. 44, 2615-2622 (2013)

92. T.F. Cao, L. Zhou, X.H. Huang, L. Wang, D.K. Wang, Q. Wu, W.Y. Hou, Chin. Vet. Med. Mag. 33, 31-33 (2014)

93. J. He, C.D. Zhou, B.Z. Ma, F. Liu, X. Liu, T. Zhao, Chin. Pharm. 26, 4433-4436 (2015)

94. H. Huang, J. Zhou, Food Ind. Tech. 30, 315-318 (2009)

95. L. An, J.T. Tang, X.M. Liu, N.N. Gao, Chin. J. of Chin. Mat. Med. 31(1225-1226), 1260 (2006)

96. G. Butt, M.A. Romero, F. Tahir, A.A. Farooqi, J. of Cell. Biochem. 119, 9640-9644 (2018)

97. X. Zhang, Z. Yan, T. Xu, Z. An, W. Chen, F. Zhu, Onc. Lett. 15, 6329-6335 (2018)

98. S.E. Potawale, S.D. Sinha, K.K. Shroff, H.J. Dhalawat, S.S. Boraste, S.P. Gandhi, A.D. Tondare, Pharmacol. Onl. 3, 140-163 (2008)

99. K. Aftab, R. Noreen, S.A. Bukhari, A. Malik, A. Sahar, Oxid Commun. 39, 3079-3089 (2016)

100. Z. Yousaf, Y. Wang, E. Baydoun, J. App. Pharm. Sci. 3, 152-160 (2013)

101. J. Lachman, K. Hamouz, M. Orsak, V. Pivec, Rost. Vyr. 47, 181-191 (2001)

102. Y. Zhou, Z.S. Deng, F. Cheng, W.J. Dong, K. Zou, Chem. Nat. Comp. 52, 920-921 (2016)

103. F. Cheng, X. Li, J.Z. Wang, Chin. Chem. Lett. 19, 68-70 (2008)

104. K. Yushan, S. Huang, M. Xie, Y. Xue, J. Zou, Med. Plant 6, 1-3 (2015)

105. Y.S. Kong, S.L. Huang, M.X. Xie, Y.H. Xue, K. Zou, S.P. Liu, Nat. Prod. Res. Dev. 26, 943-946 (2014)

106. S.L. Huang, H.B. He, K. Zou, C.H. Bai, Y.H. Xue, J.Z. Wang, J.F. Chen, J. Pharm. Pharmacol. 66, 844-854 (2014)

107. L.C. Lopes, J.E. de Carvalho, M. Kakimore, C.D.B. Vendramini, Inflammopharmacology 22, 179-185 (2014)

108. R. Grando, M.A. Antonio, C.E.P. Araujo, C. Soares, M.A. Medeiros, J. Biosci. 63, 507-514 (2008)

109. A.M. Mariza, M. Lemos, A.C. Kamila, D. Rodenburg, J.D. McChesney, J. Ethnopharmacol. 172, 421-429 (2015)

110. J.L. Damasceno, P.F. Oliveira, M.A. Miranda, L.F. Leandro, Biomed. Pharmacother. 83, 1111-1115 (2016)

111. L.C. Lopes, B. Roman, M.A. Medeiros, A. Mukhopadhyay, Tetrahedron Lett. 52, 6392-6395 (2011)

112. J.L. Damasceno, P.F. de Oliveira, M.A. Miranda, M. Lima, C. Denise, Biol. Pharm. Bull. 39, 920-926 (2016)

113. A. Herrera, F.E. Jimenez, A. Zamilpa, R.M.A. Martinez, Planta Med. 75, 466-471 (2009)

114. H.A. Armando, J.F. Enrique, V.P.A. Maria, Planta Med. 70, 483-488 (2004) 
115. H.A. Armando, V.E.O. Lopez, T.A.V. Rodriguez, A. Zamilpa, R.M.A. Martinez, Afr. J. Trad. Comp. Altern. Med. 10, 410-417 (2013)

116. H.A. Armando, R.S. Artemio, M.R. Maria, M.C. Eugenia, J. Tortoriello, Planta Med. 69, 390-395 (2003)

117. A. Zamilpa, J. Tortoriello, V. Navarro, G. Delgado, L. Alvarez, J. Nat. Prod. 65, 1815-1819 (2002)

118. L. Alvarez, M.D.C. Perez, J.L. Gonzalez, V. Navarro, M.L. Villarreal, Planta Med. 67, 372-374 (2001)

119. X. Lozoya, V. Navarro, M. Garcia, M. Zurita, J. Ethnopharmacol. 36, 127-132 (1992)

120. L. Alvarez, H.A. Armando, S. Marquina, J. Tortoriello, A. Zamilpa, V. Navarro, Curr. Top. Steroid Res. 6, 89-104 (2009)

121. I. Gaitaan, A.M. Paz, S.A. Zacchino, G. Tamayo, A. Gimeenez, R. Pinzoon, Pharm. Biol. 49, 907-919 (2011)

122. M.T.F. Cornelius, M.G. de Carvalho, T.M.S. da Silva, C.C.F. Alves, J. Braz. Chem. Soc. 21, 2211-2219 (2010)

123. E.S. Andressa, T.M.S. da Silva, C.C.F. Alves, M.G. de Carvalho, J. Braz. Chem. Soc. 13, 838-842 (2002)

124. https://en.wikipedia.org/wiki/Solanum_diphyllum. Accessed 10 Sept 2017

125. https://toptropicals.com/catalog/uid/Solanum_diphyllum.htm. Accessed 10 Sept 2017

126. M.A. El-Sayed, A.E.H. Mohamed, M.K. Hassan, M.E.F. Hegazy, J. Biosci. 64, 644-649 (2009)

127. https://www.fs.fed.us/database/feis/plants/shrub/soldul/all.html

128. https://en.wikipedia.org/wiki/Solanum_dulcamara. Accessed 10 Sept 2017

129. T. Yamashita, T. Matsumoto, S. Yahara, N. Yoshida, T. Nohara, Chem. Pharm. Bull. 39, 1626-1628 (1991)

130. T. Sabudak, O. Kaya, E. Cukurova, Nat. Prod. Res. 29, 308-314 (2015)

131. N. Mimica-Dukic, L. Krstic, P. Boza, Oxid. Commun. 28, 536546 (2005)

132. H. Tunon, H.C. Olavsdotter, L. Bohlin, J. Ethnopharmacol. 48, 61-76 (1995)

133. https://en.wikipedia.org/wiki/Solanum_elaeagnifolium. Accessed 13 Sept 2017

134. H. Feki, I. Koubaa, H. Jaber, J. Makni, M. Damak, J. Eng. Appl. Sci. 8, 708-712 (2013)

135. http://www.cabi.org/isc/datasheet/120139. Accessed 14 Sept 2017

136. https://en.wikipedia.org/wiki/Solanum_erianthum. Accessed 14 Sept 2017

137. S.C. Chou, T.J. Huang, E.H. Lin, C.H. Huang, C.H. Chou, Nat. Prod. Commun. 7, 153-156 (2012)

138. S.Y. Peng, H. Li, D. Yang, B. Bai, L.P. Zhu, Q. Liu, Nat. Prod. Res. 31, 810-816 (2017)

139. S.D. Priyadharshini, V. Sujatha, Int. J. Pharm. Pharm. Sci. 5, 652-658 (2013)

140. M.A. Monem, R. Azza, Bull. Fac. Pharm. 47, 59-66 (2009)

141. A.S. Essam, M.A. Farag, E.A. Mahrous, Rec. Nat. Prod. 9, 94-104/1 (2015)

142. N.A. Siddiqui, M.A. Parvez, A.J. Al-Rehaily, M.S. Al Dosari, Saudi Pharm. J. 25, 184-195 (2017)

143. R.A. Mothana, N.M. Al-Musayeib, M.F. Al-Ajmi, P. Cos, L. Maes, Evidence-based Comp. Altern. Med. 2014, 905639 (2014)

144. R.A.A. Mothana, R. Gruenert, P.J. Bednarski, U. Lindequist, Pharmazie 64, 260-268 (2009)

145. https://en.wikipedia.org/wiki/Solanum_glaucophyllum. Accessed 14 Sept 2017

146. C.N. Zanuzzi, F. Nishida, E.L. Portiansky, P.A. Fontana, E.J Gimeno, C.G. Barbeito, Res. Vet. Sci. 93, 336-342 (2012)

147. M. Zadra, M. Piana, T. de Brum, F. Thiele, A.A. Boligon, Molecules 17, 12560-12574 (2012)
148. G. Bonfanti, K.S.D. Bona, L.D. Lucca, L. Jantsch, M.B. Moretto, Red. Rep. 19, 206-213 (2014)

149. G. Bonfanti, P.R. Bitencourt, S.B. Karine, S.S. Priscila, Molecules 18,9179-9194 (2013)

150. https://en.wikipedia.org/wiki/Solanum_incanum. Accessed 14 Sept 2017

151. http://keys.lucidcentral.org/keys/v3/eafrinet/weeds/key/weeds/ Media/Html/Solanum_incanum_(Sodom_Apple).htm. Accessed 14 Sept 2017

152. S.A. Alamri, M.F. Moustafa, Saudi Med. J. 33, 272-277 (2012)

153. B. Taye, M. Giday, A. Animut, J. Seid, Asian Pac. J. Trop. Biomed. 1, 370-375 (2011)

154. S.S. Al-Sokari, N.A.A. Ali, L. Monzote, M. Al-Fatimi, Biomed. Res. Int. 2015, 938747 (2015)

155. M.J. Manase, O.A.C. Mitaine, D. Pertuit, T. Miyamoto, C. Tanaka, S. Delemasure, Fitoterapia 83, 1115-1119 (2012)

156. S. Sundar, Y.J.K. Pillai, Asian J. Pharm. Clin. Res. 8, 179-188 (2015)

157. https://easyayurveda.com/2014/06/20/brihati-solanum-indicumqualities-benefits-dose-side-effect/. Accessed 25 Sept 2017

158. http://www.geniusherbs.com/solanum-indicum.html. Accessed 25 Sept 2017

159. Y.W. Zhuang, C.E. Wu, J.Y. Zhou, Z.M. Zhao, C.L. Liu, S.L. Liu, Biochem. Biophy. Res. Commun. 505, 485-491 (2018)

160. H.C. Chiang, T.H. Tseng, C.J. Wang, C.F. Chen, W.S. Kan, Anticancer Res. 11, 1911-1917 (1991)

161. A. Aberoumand, S.S. Deokule, J. Food Technol. 8, 131-133 (2010)

162. P. Ma, T.T. Cao, G.F. Gu, X. Zhao, Y.G. Du, Y. Zhang, Chin. J. Cancer 25, 438-442 (2006)

163. W.J. Syu, M.J. Don, G.H. Lee, C.M. Sun, J. Nat. Prod. 64, 1232 $1233(2001)$

164. W.H. Huang, C.W. Hsu, J.T. Fang, Clin. Toxicol. 46, 293-296 (2008)

165. https://link.springer.com/article/10.2307/2807835. Accessed 26 Sept 2017

166. T.M.S. Silva, R. Braz-Filho, M.G. de Carvalho, M.F. Agra, Biochem. Syst. Ecol. 30, 1083-1085 (2002)

167. T.M.S. Silva, G.M. de Carvalho, B.F. Raimundo, Quim. Nova 32, 1119-1128 (2009)

168. E.S. Andressa, T.M.S.S. da Silva, C.C.F. Alves, M.G. de Carvalho, A. Echevarria, Aurea J. Braz. Chem. Soc. 13, 838-842 (2002)

169. T.M.S. Silva, C.A. Camara, M.F. Agra, M.G. de Carvalho, B.F. Raimundo, Fitoterapia 77, 449-452 (2006)

170. E.E. Jarald, S. Edwin, V. Saini, L. Deb, V.B. Gupta, S.P. Wate, K.P. Busari, Nat. Prod. Res. 22, 267-274 (2008)

171. R. Chand, Indian Drugs 30, 650 (1993)

172. G. Rosangkima, G.C. Jagetia, J. Pharm. Res. 4, 98-103 (2015)

173. http://www.plantoftheweek.org/week351.shtml. Accessed 27 Sept 2017

174. http://www.anbg.gov.au/gnp/gnp12/solanum-laciniatum.html. Accessed 27 Sept 2017

175. https://en.wikipedia.org/wiki/Solanum_laxum. Accessed 27 Sept 2017

176. https://plantsam.com/solanum-laxum/. Accessed 27 Sept 2017

177. S. Soule, C. Guntner, A. Vazquez, V. Argandona, P. Moyna, F. Ferreira, Phytochemistry 55, 217-222 (2000)

178. F. Ferreira, S. Soule, A. Vazquez, P. Moyna, L. Kenne, Phytochemistry 42, 1409-1416 (1996)

179. C. Delporte, N. Backhouse, R. Negrete, P. Salinas, P. Rivas, B.K. Cassels, Phytother. Res. 12, 118-122 (1998)

180. C.C. Munari, P.F. Oliveira, J.C.L. Campos, S.P.L. Martins, J. Nat. Med. 68, 236-241 (2014)

181. C.C. Munari, P.F. de Oliveira, L.F. Leandro, L.M. Pimenta, N.H. Ferreira, PLoS ONE 9, 111999 (2014) 
182. M. Yoshikawa, S. Nakamura, K. Ozaki, A. Kumahara, T. Morikawa, H. Matsuda, J. Nat. Prod. 70, 210-214 (2007)

183. M.A. Miranda, L.G.M. Magalhaes, R.F.J. Tiossi, C.C. Kuehn, Parasit. Res. 111, 257-262 (2012)

184. A.M. Mariza, C.C. Kuehn J.F.R. Cardoso, L.G.R. Oliveira, L.G. Magalhaes, Exp. Parasit. 133, 396-402 (2013)

185. A.M. Mariza, R.F.J. Tiossi, M.R. da Silva, K.C. Rodrigues, C.C. Kuehn, Chem. Biodiversity 10, 642-648 (2013)

186. R.R.D. Moreira, G.Z. Martins, N.O. Magalhaes, A.E. Almeida, R.C.L.R. Pietro, Anais Acad. Bras. Cienc. 85, 903-907 (2013)

187. G.Z. Martins, R.R.D. Moreira, C.S. Planeta, A.E. Almeida, J.K. Bastos, Pharmacogn. Mag. 11, 161-165 (2015)

188. https://en.wikipedia.org/wiki/Tomato. Accessed 03 Oct 2017

189. M. Kralova, M. Sanda, M. Mackova, T. Macek, Collec. Symp. Series 13, 73-76 (2011)

190. S. Paoli, A.P.M. Dias, P.V.S.Z. Capriles, T.E.M.M. Costa, Rev. Bras. Farmacogn. 18, 190-196 (2008)

191. E. Fuentes, R. Castro, L. Astudillo, G. Carrasco, Evidencebased Comp. Altern. Med. 2012, 147031 (2012)

192. M. Friedman, C.E. Levin, S.U. Lee, H.J. Kim, I.S. Lee, J. Agric. Food Chem. 57, 5727-5733 (2009)

193. http://www.fpen.net/a/guanmu/20131109/Solanum_lyrat um.html. Accessed 07 Oct 2017

194. L.X. Sun, W.W. Fu, J. Ren, L. Xu, K.S. Bi, M.W. Wang, Arch. Pharm. Res. 29, 135-139 (2006)

195. S.H. Liu, X.H. Shen, X.F. Wei, X.H. Mao, T. Huang, Immunopharmcol. Immunotoxicol. 33, 100-106 (2011)

196. X.P. Nie, F. Yao, X.D. Yue, G.S. Li, S.J. Dai, Nat. Prod. Res. 28, 641-645 (2014)

197. G.S. Li, F. Yao, L. Zhang, X.D. Yue, S.J. Dai, Chin. Chem. Lett. 24, 1030-1032 (2013)

198. F. Yao, Q.L. Song, L. Zhang, G.S. Li, S.J. Dai, Fitoterapia 89, 200-204 (2013)

199. Y. Ren, L. Shen, D.W. Zhang, S.J. Dai, Chem. Pharm. Bull. 57, 408-410 (2009)

200. F. Yao, Q.L. Song, L. Zhang, G.S. Li, S.J. Dai, Phytochem. Lett. 6, 453-456 (2013)

201. D.W. Zhang, Y. Yang, F. Yao, Q.Y. Yu, S.J. Dai, J. Nat. Med. 66, 362-366 (2012)

202. https://en.wikipedia.org/wiki/Eggplant. Accesed 02 Nov 2017

203. https://www.thoughtco.com/eggplant-history-solanum-melon gena-170820. Accessed 02 Nov 2017

204. M.A.S. Atta, M.T.K. Shahid, Oxid. Commun. 39, 2249-2259 (2016)

205. A.P. Singh, D. Luthria, T. Wilson, N. Vorsa, V. Singh, Food Chem. 114, 955-961 (2009)

206. A. Zhao, Y. Sakurai, K. Shibata, F. Kikkawa, Y. Tomoda, H. Mizukami, Nippon Shokuhin Kagaku Gakkaish 21, $42-47$ (2014)

207. M.M. Shabana, M.M. Salama, S.M. Ezzat, L.R. Ismail, J. Carcinog. Mutagen. 4, 1000149/1-1000149/6 (2013)

208. K. Yoshikawa, K. Inagaki, T. Terashita, J. Shishiyama, S. Kuo, D.M. Shankel, Mutagen. Res. Gen. Toxicol. 371, 65-71 (1996)

209. X. Liu, J. Luo, L. Kong, Nat. Prod. Commun. 6, 851-853 (2011)

210. J. Sun, Y.F. Gu, X.Q. Su, M.M. Li, H.X. Huo, J. Zhang, Fitoterapia 98, 110-116 (2014)

211. A. Nishina, K. Ebina, M. Ukiya, M. Fukatsu, M. Koketsu, M. Ninomiya, J. Food Sci. 80, H2354-H2359 (2015)

212. W. Ren, D.G. Tang, Antican. Res. 19, 403-408 (1999)

213. F.J. Herraiz, M. Plazas, S. Vilanova, J. Prohens, D. Villano, F. Ferreres, Int. J. Mol. Sci. 17, 394 (2016)

214. V.H.C. Chang, T.H. Chiu, S.C. Fu, J. Sci. Food Agric. 96, 192$198(2016)$

215. C.C. Hsu, Y.R. Guo, Z.H. Wang, M.C. Yin, J. Sci. Food Agric. 91, 1517-1522 (2011)

216. http://www.efloras.org/florataxon.aspx?flora_id=601\&taxon _id=200020596. Accessed 07 Nov 2017
217. http://www.nhm.ac.uk/natureplus/blogs/china/2010/03/06/thesearch-for-solanum-nienkui?fromGateway $=$ true. Accessed 07 Nov 2017

218. https://www.wikidata.org/wiki/Q15239976. Accessed 07 Nov 2017

219. https://en.wikipedia.org/wiki/Solanum_nigrum. Accessed 08 Nov 2017

220. http://naturalhomeremedies.co/Snigrum.html. Accessed 08 Nov 2017

221. https://baike.baidu.com/item/\%E9\%BE\%99\%E8\%91\%B5\%E $8 \% 8 \mathrm{~A} \% \mathrm{~B} 1 / 9378068 ? \mathrm{fr}=$ aladdin $\&$ fromid $=11201243 \&$ fromt itle=Solanum+nigrum. Accessed 08 Nov 2017

222. http://www.pfaf.org/user/Plant.aspx ? Latin Name=Solan um+nigrum. Accessed 08 Nov 2017

223. B.K. Sharma, D. Iyer, U.K. Patil, J. Herb. Spic. Med. Plants 18, 257-267 (2012)

224. T.M. Sridhar, P. Josthna, C.V. Naidu, J. Exp. Sci. 2, 24-29 (2011)

225. L.G. Matasyoh, H.M. Murigi, J.C. Matasyoh, Afr. J. Microbiol. Res. 8, 3923-3930 (2014)

226. F.Z. Khan, M.A. Saeed, M. Alam, A.R. Chaudhry, M. Ismail, J. Fac. Pharm. Gazi Uni. 10, 105-116 (1993)

227. H.L. Yuan, X.L. Liu, Y.J. Liu, Asian Pac. J. Cancer Prev. 15 , 10469-10473 (2014)

228. X. Ding, F. Zhu, Y. Yang, M. Li, Food Chem. 141, 1181-1186 (2013)

229. K.V. Prashanth, S. Shashidhara, M.M. Kumar, B.Y. Sridhara, Fitoterapia 72, 481-486 (2001)

230. H.C. Huang, K.Y. Syu, J.K. Lin, J. Agric. Food Chem. 58, 86998708 (2010)

231. G.T. El-Sherbini, R.A. Zayed, E.T. El-Sherbini, J. Parasit. Res. 2009, 474360 (2009)

232. H. Hammami, A. Ayadi, J. Helminth. 82, 235-239 (2008)

233. A. Rawani, A.S. Ray, G. Chandra, A. Ghosh, M. Sakar, BMC Res. Notes 10, 135 (2017)

234. Z.A. Zakaria, H.K. Gopalan, H. Zainal, P.N.H. Mohd, Yakugaku Zasshi 126, 1171-1178 (2006)

235. M. Jainu, C.S.S. Devi, J. Ethnopharmacol. 104, 156-163 (2006)

236. H.M. Lin, H.C. Tseng, C.J. Wang, J.J. Lin, C.W. Lo, Chem.-Biol. Int. 171, 283-293 (2008)

237. C.C.H.L. Fang, W.C. Lina, J. Ethnopharmacol. 119, 117-121 (2008)

238. R.M. Perez, J.A. Perez, L.M. Garcia, J. Ethnopharmacol. 62, 43-48 (1998)

239. http://eol.org/pages/5695318/overview. Accessed 10 Nov 2017

240. A. Pabon, S. Blair, J. Carmona, M. Zuleta, J. Saez, Pharm. 58, 263-267 (2003)

241. G. Alvarez, A. Pabon, J. Carmona, S. Blair, Phytother. Res. 18 , 845-848 (2004)

242. M.L. Lopez, R. Vommaro, M. Zalis, W. Souza, S. Blair, C. Segura, Parasit. Int. 59, 217-225 (2010)

243. M.L. Lopez, S. Blair, J. Saez, C. Segura, Memo. Inst. Oswaldo Cruz 104, 683-688 (2009)

244. A. Pabon, E. Deharo, L. Zuluaga, J.D. Maya, J. Saez, S. Blair, Exp. Parasit. 122, 273-279 (2009)

245. B. Londono, E. Arango, C. Zapata, S. Herrera, J. Saez, S. Blair, F.J. Carmona, Phytother. Res. 20, 267-273 (2006)

246. O.M.S. Cardoso, E.J.N. Gomez, L.A. Garces, S.B. Trujillo, Rev. Colomb. Biotechnol. 13, 186-192 (2011)

247. J. Saez, W. Cardona, D. Espinal, S. Blair, J. Mesa, M. Bocar, A. Jossang, Tetrahedron 54, 10771-10778 (1998)

248. G.H. Paola, A. Pabon, C. Arias, S. Blair, Biomed. 33, 78-87 (2013)

249. M. Echeverri, S. Blair, J. Carmona, P. Perez, Am. J. Chin. Med. 29, 477-484 (2001)

250. R.M. Coelho, M.C. Souza, M.H. Sarragiotto, Phytochemistry 49, 893-897 (1998) 
251. https://commons.wikimedia.org/wiki/Category:Solanum_palud osum. Accessed 14 Nov 2017

252. F.S. Monteiro, A.C.L. Silva, I.R.R. Martins, A.C.C. Correia, J. Ethnopharmacol. 141, 895-900 (2012)

253. M.L.C. Valverde, J. Boustie, H.E. Badaoui, B. Muguet, M. Henry, Planta Med. 59, 483-484 (1993)

254. https://en.wikipedia.org/wiki/Solanum_paniculatum. Accessed 14 Nov 2017

255. http://rain-tree.com/jurubeba.htm\#.WgqWOrVx3IU. Accessed 14 Nov 2017

256. http://www.pfaf.org/user/Plant.aspx?Latin Name=Solan um+paniculatum. Accessed 14 Nov 2017

257. Y.M. Valadares, G.C. Brandao'a, E.G. Kroon, J.D.S. Filho, A.B. Oliveira, F.C. Braga, J. Biosci. 64, 813-818 (2009)

258. G.M.J. Vieira, Q.C. Rocha, R.T. Souza, C.L.H. Lima, W. Vilegas, Food Chem. 186, 160-167 (2015)

259. V.S. Mesia, M.T. Santos, C. Souccar, L.M.T.R. Lima, A.J. Lapa, Phytomed. Int. J. Phytother. Phytopharm. 9, 508-514 (2002)

260. A.B. Valerino-Diaz, G.T. Daylin, A.C. Zanatta, W. Vilegas, L. dos Santos, J. Agric. Food Chem. 66, 8703-8713 (2018)

261. https://en.wikipedia.org/wiki/Solanum_pseudocapsicum. Accessed 14 Nov 2017

262. https://davesgarden.com/guides/pf/go/54393/. Accessed 14 Nov 2017

263. A.A. Aliero, O.T. Asekun, D.S. Grierson, A.J. Afolayan, Asian J. Plant Sci. 5, 1054-1056 (2006)

264. P. Vijayan, H.C. Prashanth, P. Vijayaraj, S.A. Dhanaraj, S. Badami, B. Suresh, Pharm. Biol. 41, 443-448 (2003)

265. https://en.wikipedia.org/wiki/Solanum_rostratum. Accessed 15 Nov 2018

266. https://fireflyforest.net/firefly/2006/07/24/buffalobur-nightshade -a-very-evil-plant/. Accessed 15 Nov 2018

267. https://plants.usda.gov/core/profile?symbol=soro. Accessed 15 Nov 2018

268. https://www.cabi.org/isc/datasheet/50544. Accessed 15 Nov 2018

269. https://www.sciencedirect.com/topics/agricultural-and-biological -sciences/solanum-rostratum. Accessed 15 November 2018

270. http://ucjeps.berkeley.edu/eflora/eflora_display.php?tid=44902. Accessed 15 Nov 2018

271. https://www.illinoiswildflowers.info/weeds/plants/buffalo_bur. html. Accessed 15 Nov 2018

272. https://www.invasiveplantatlas.org/subject.html? sub $=6463$. Accessed 15 Nov 2018

273. C.A. Ibarra, A. Rojas, S. Mendoza, M. Bah, D.M. Gutierrez, S.L. Hernandez, M. Martinez, Pharmaceu. Bio. 48, 732-739 (2010)

274. https://en.wikipedia.org/wiki/Solanum_sarrachoides

275. https://www.apsnet.org/publications/plantdisease/2002/May/ Pages/86_5_559.3.asx

276. http://plants.jstor.org/compilation/solanum.schimperianum. Accessed 15 Nov 2017

277. W.H.B. Hassan, M. Al-Oqail, M.S. Ahmad, A.J. Al-Rehaily, Biosci. Biotechnol. Res Asia 9, 593-599 (2012)

278. W.H.B. Hassan, M. Al-Oqail, M.S. Ahmad, A.J. Al-Rehaily, Saudi Pharm. J. 20, 371-379 (2012)

279. A.J. Al-Rehaily, J. Adnan, M.S. Ahmad, J. Mustafa, M.M. AlOqail, I.A. Khan, J. Saudi Chem. Soc. 17, 67-76 (2013)

280. http://plants.for9.net/edible-and-medicinal-plants/solanumseptemlobum/. Accessed 15 Nov 2017

281. L. Zhang, G.S. Li, F. Yao, X.D. Yue, S.J. Dai, Phytochem. Lett. 11, 173-176 (2015)

282. https://en.wikipedia.org/wiki/Solanum_sessiliflorum. Accessed 15 Nov 2017

283. https://www.hort.purdue.edu/newcrop/morton/cocona.html. Accessed 15 Nov 2017
284. http://www.tradewindsfruit.com/content/cocona.htm. Accessed 15 Nov 2017

285. J.R.P. Maia, M.C. Schwertz, R.F.S. Sousa, J.P.L. Rev, Bras. Plantas Med. 17, 112-119 (2015)

286. https://en.wikipedia.org/wiki/Solanum_sisymbriifolium. Accessed 15 Nov 2017

287. http://www.jeremybartlett.co.uk/2014/10/19/sticky-nightshade -solanum-sisymbriifolium/. Accessed 15 Nov 2017

288. http://www.pfaf.org/user/Plant.aspx? Latin Name=Solan um+sisymbriifolium. Accessed 15 Nov 2017

289. D.A. Ibarrola, M.D.C. Hellion-Ibarrola, N.L. Alvarenga, Pharm. Biol. 44, 378-381 (2006)

290. A.S. Apu, S.H. Bhuyan, M. Matin, F. Hossain, F. Khatun, A. Taiab Abu, M. Matin, Avicenna J. Phytomed. 3, 302-312 (2013)

291. D.A. Ibarrola, M.C.H. Ibarrola, Y. Montalbetti, O. Heinichen, M.A. Campuzano, Phytomedicine 18, 634-640 (2011)

292. D.A. Ibarrola, M.C.I. Hellion, Y. Montalbetti, O. Heinichen, N. Alvarenga, J. Ethnopharmacol. 70, 301-307 (2000)

293. V.K. Gupta, A. Simlai, M. Tiwari, K. Bhattacharya, A. Roy, J. Appl. Pharm. Sci. 4, 75-80 (2014)

294. K. Chauhan, N. Sheth, V. Ranpariya, S. Parmar, Pharm. Biol. 49, 194-199 (2011)

295. J.J.M. Bagalwa, V.N. Laurence, C. Sayagh, A.S. Bashwira, Fitoterapia 81, 767-771 (2010)

296. T.O. Siddiqi, J. Ahmad, S.U. Khan, K. Javed, M.S.Y. Khan, Philipp. J. Sci. 119, 41-47 (1990)

297. S. Keawsa-ard, S. Natakankitkul, S. Liawruangrath, A. Teerawutgulrag, Chiang Mai J. Sci. 39, 445-454 (2012)

298. K.A. Sukanya, B. Liawruangrath, S. Liawruangrath, A. Teerawutgulrag, Nat. Prod. Commun. 7, 955-958 (2012)

299. T. Payum, A.K. Das, R. Shankar, C. Tamuly, M. Hazarika, Am. J. PharmTech Res. 5, 307-314 (2015)

300. http://www.zhiwutong.com/latin/Solanaceae/Solanum-surattense -Burm-F.htm. Accessed 29 Nov 2017

301. http://frps.eflora.cn/frps/Solanum\%20surattense. Accessed 29 Nov 2017

302. https://easyayurveda.com/2014/04/19/kantakari-solanum-surat tense-benefits-dose-usage-side-effects/. Accessed 29 Nov 2017

303. S.A. Patil, S.N. Sambrekar, Int. J. Res. Pharma. Biomed. Sci. 3, 1559-1566 (2012)

304. T. Ahmed, R. Kanwal, N. Ayub, M. Hassan, Hum. Ecol. Risk Assess. 15, 624-635 (2009)

305. Y. Lu, J. Luo, L. Kong, Phytochemistry 72, 668-673 (2011)

306. M. Qasim, Z. Abideen, M.Y. Adnan, S. Gulzar, B. Gul, M. Rasheed, S. Afr. J. Bot. 110, 240-250 (2017)

307. A. Yadav, R. Bhardwaj, R.A. Sharma, Int. J. Pharm. Pharm. Sci. 5, 489-493 (2013)

308. M.M. Ahmed, S. Andleeb, F. Saqib, B.A. Ch, M. Hussain, M.N. Khatun, H. Rahman, B.M.C. Comp, Altern. Med. 16, 166 (2016)

309. A. Ramazani, S. Zakeri, S. Sardari, N. Khodakarim, N.D. Djadidt, Malaria J. 9, 124 (2010)

310. https://en.wikipedia.org/wiki/Solanum_torvum. Accessed 6 Dec 2017

311. http://fleppc.org/ID_book/solanum\%20torvum.pdf. Accessed 6 Dec 2017

312. http://tropical.theferns.info/viewtropical.php?id=Solanum+torvu m. Accessed 6 Dec 2017

313. W.H. Maser, N.D. Yuliana, N. Andarwulan, J. Liq. Chrom. Rel. Technol. 38, 1230-1235 (2015)

314. S.B. Paul, M.D. Choudhury, R. Choudhury, Asian J. Chem. 21, 581-588 (2009)

315. C. Balachandran, N. Emi, Y. Arun, Y. Yamamoto, B. Ahilan, B. Sangeetha, Chem.-Biol. Inter. 242, 81-90 (2015)

316. Y. Lu, J. Luo, X. Huang, L. Kong, Steroids 74, 95-101 (2009) 
317. J. Li, L. Zhang, C. Huang, F. Guo, Y. Li, Fitoterapia 93, 209-215 (2014)

318. R.U. Abhishek, S. Thippeswamy, K. Manjunath, D.C. Mohana, J. Appl. Microbiol. 119, 1624-1636 (2015)

319. K.F. Chah, K.N. Muko, S.I. Oboegbulem, Fitoterapia 71, 187189 (2000)

320. C. Balachandran, V. Duraipandiyan, N.A. Al-Dhabi, K. Balakrishna, Indian J. Microbiol. 52, 676-681 (2012)

321. T.B. Nguelefack, C.B. Feumebo, G. Ateufack, P. Watcho, S. Tatsimo, J. Ethnopharmacol. 119, 135-140 (2008)

322. D. Arthan, J. Svasti, P. Kittakoop, D. Pittayakhachonwut, M. Tanticharoen, Phytochemistry 59, 459-463 (2002)

323. S. Challal, O.E.M. Buenafe, E.F. Queiroz, S. Maljevic, L. Marcourt, M. Bock, A.C.S. Chem, Neuroscience 5, 993-1004 (2014)

324. T.B. Nguelefack, H. Mekhfi, T. Dimo, S. Afkir, M. Nguelefack, P. Elvine, A. Legssyer, A. Ziyyat, J. Comp. Integ. Med. 5, (2008)

325. M. Mohan, B.S. Jaiswal, S. Kasture, J. Ethnopharmacol. 126, 86-89 (2009)

326. M. Mohan, S. Kamble, P. Gadhi, S. Kasture, F. Chem, Toxicology 48, 436-440 (2010)

327. C.H. Ramamurthy, A. Subastri, A. Suyavaran, C. Thirunavukkarasu, Environ. Sci. Pollut. Res. Int. 23, 7919-7929 (2016)

328. C. Lalmuanthanga, C. Lalchhandama, M.C. Lallianchhunga, M.A. Ali, World J. Pharm. Res. 4, 1752-1759 (2015)

329. W. Kusirisin, C. Jaikang, C. Chaiyasut, P. Narongchai, Med. Chem. 5, 583-588 (2009)

330. C.H. Ramamurthy, M.S. Kumar, V.S.A. Suyavaran, J. Food Sci. 77, 907-913 (2012)

331. C.L. Lee, T.L. Hwang, W.J. He, Y.H. Tsai, C.T. Yen, H.F. Yen, C.J. Chen, Phytochemistry 95, 315-321 (2013)

332. M. Mohan, D. Attarde, R. Momin, S. Kasture, Nat. Prod. Res. 27, 2140-2143 (2013)

333. R. Momin, M. Mohan, Nat. Prod. Res. 26, 416-422 (2012)

334. C. Kamaraj, N.K. Kaushik, D. Mohanakrishnan, G. Elango, A. Bagavan, Parasit. Res. 111, 703-715 (2012)

335. G.R. Gandhi, S. Ignacimuthu, M.G. Paulraj, P. Sasikumar, Eur. J. Pharmacol. 670, 623-631 (2011)

336. G.R. Gandhi, S. Ignacimuthu, M.G. Paulraj, Food Chem. Toxicol. 49, 2725-2733 (2011)

337. K. Takahashi, Y. Yoshioka, E. Kato, S. Katsuki, K. Hosokawa, J. Kawabata, Biosci. Biotechnol. Biochem. 74, 741-745 (2010)

338. C. Kamaraj, A.A. Rahuman, G. Elango, A. Bagavan, A.A. Zahir, Parasit. Res. 109, 37-45 (2011)

339. http://swbiodiversity.org/seinet/taxa/index.php?taxon $=28414$. Accessed 19 Dec 2017

340. http://www.conabio.gob.mx/malezasdemexico/solanaceae/solan um-tridynamum/fichas/ficha.htm. Accessed 19 Dec 2017

341. L. Brito, F. Wendy, S. Mejia, M. Gaspar, C.M. Francisco, Afinidad 52, 49-52 (1995)

342. http://tropical.theferns.info/viewtropical.php?id=Solanum+trilo batum. Accessed 21 Dec 2017

343. http://naturalhomeremedies.co/Strilobatum.html. Accessed 21 Dec 2017

344. A. Kanchana, C. Panneerselavam, Int. J. Curr. Res. Rev. 3, 37-51 (2011)

345. R.R. Thanigaiarassu, K. Kannabiran, V.G. Khanna, J. Pharm. Res. 2, 273-276 (2009)

346. K. Kannabiran, R.R. Thanigaiarassu, G.K. Venkatesan, J. Appl. Biol. Sci. 2, 109-112 (2008)

347. M. Vanaja, K. Paulkumar, G. Gnanajobitha, S. Rajeshkumar, C. Malarkodi, G. Annadurai, (International Journal of Metals 2014), pp. 1-9

348. A.K. Zameer, S.S.Z. Ahmed, P. Ponnusamy, K.B. Senthil, Pak. J. Pharm. Sci. 29, 1578 (2016)

349. M.B. Kanchana, Int. J. Pharm. Pharm. Sci. 3, 356-364 (2011)

350. P.V. Mohanan, K.S. Devi, Cancer Lett. 110, 71-76 (1996)
351. P.V. Mohanan, K.S. Devi, Cancer Lett. 112, 219-223 (1997)

352. M. Shahjahan, G. Vani, C.S. Shyamaladevi, Chem.-Biol. Int. 156, 113-123 (2005)

353. P. Jagadeesan, D.A. Prasad, P. Pandikumar, S. Ignacimuthu, Indian J. Nat. Prod. Res. 2, 156-163 (2011)

354. P.V. Mohanan, K.S. Devi, J. Exp. Clin. Cancer Res. 17, 159-164 (1998)

355. P. Govindarajan, C. Chinnachamy, Pak. J. Pharm. Sci. 27, 2101 2107 (2014)

356. P.N. Venkatesan, P. Rajendran, G. Ekambaram, D. Sakthisekaran, Nat. Prod. Res. 22, 1094-1106 (2008)

357. S. Rajkumar, A. Jebanesan, J. Ins. Sci. 5, 15 (2005)

358. A. Pandurangan, R.L. Khosa, S. Hemalatha, Nat. Prod. Res. 25, 1132-1141 (2011)

359. A. Pandurangan, R.L. Khosa, S. Hemalatha, J. Asian Nat. Prod. Res. 12, 691-695 (2010)

360. M. Shahjahan, K.E. Sabitha, M. Jainu, D.C.S. Shyamala, Indian J. Med. Res. 120, 194-198 (2004)

361. http://solanaceaesource.org/taxonomy/term/110074/descriptions. Accessed 21 Dec 2017

362. A. Maxwell, M. Seepersaud, R. Pingal, D.R. Mootoo, W.F. Reynolds, J. Nat. Prod. 59, 200-201 (1996)

363. https://en.wikipedia.org/wiki/Potato. Accessed 22 Dec 2017

364. https://www.hort.purdue.edu/newcrop/duke_energy/Solanum tuberosum.html. Accessed 22 Dec 2017

365. K.D. Chandrashekara, S.M. Dharmesh, J. Pharm. Res. 8, 11481157 (2014)

366. A.A.A. Mohdaly, M.A. Sarhan, I. Smetanska, A. Mahmoud, J. Sci. Food Agric. 90, 218-226 (2010)

367. D. Paik, P. Das, T. De, T. Chakraborti, Exp. Parasit. 146, 11-19 (2014)

368. D. Paik, P. Das, P.K. Pramanik, K. Naskar, T. Chakraborti, Biomed. Pharmacother. 83, 1295-1302 (2016)

369. T. Zuber, D. Holm, P. Byrne, L. Ducreux, M. Taylor, M. Kaiser, C. Stushnoff, Food Funct. 6, 72-83 (2015)

370. E. Langner, F.M. Nunes, P. Pozarowski, M. Kandefer-Szerszen, Food Chem. Toxicol. 57, 246-255 (2013)

371. L. Reddivari, J. Vanamala, S. Chintharlapalli, S.H. Safe, J.C. Miller Jr., Carcinogenesis 28, 2227-2235 (2007)

372. M. Friedman, K.R. Lee, H.J. Kim, I.S. Lee, N. Kozukue, J. Agric. Food Chem. 53, 8420 (2005)

373. J.A.O. Ojewole, D.R. Kamadyaapa, C.T. Musabayane, Cardiovasc. J. S. Afr. 17, 166-171 (2006)

374. http://www.calflora.org/cgi-bin/species_query.cgi?where-taxon $=$ Solanum\%20umbelliferum. Accessed 23 Dec 2017

375. http://calscape.org/Solanum-umbelliferum. Accessed 23 Dec 2017

376. https://en.wikipedia.org/wiki/Solanum_umbelliferum. Accessed 23 Dec 2017

377. https://www.wildflower.org/plants/result.php?id_plant=soum. Accessed 23 Dec 2017

378. http://www.watershednursery.com/nursery/plant-finder/solanumumbelliferum/. Accessed 23 Dec 2017

379. http://www.theodorepayne.org/mediawiki/index.php?title=Solan um_umbelliferum. Accessed 23 Dec 2017

380. Y.C. Kim, Q.M. Che, A.A.L. Gunatilaka, D.G.I. Kingston, J. Nat. Prod. 59, 283-285 (1996)

381. http://www.shaman-australis.com/forum/index.php?/topic/33853 -cannibals-tomato-solanum-viride-s-uporo-s-anthropophagoru $\mathrm{m} /$. Accessed 25 Dec 2017

382. http://www.asklepios-seeds.de/gb/solanum-uporo-seeds.html. Accessed 25 Dec 2017

383. http://www.tradewindsfruit.com/content/cannibal-tomato.htm. Accessed 25 Dec 2017

384. H. Ripperger, Phytochemistry 44, 731-734 (1997) 
385. L.E.C. Suarez, D.R.M. Cendales, I.O.P. Clara, Rev. Colomb. Quimica 35, 59-65 (2006)

386. https://en.wikipedia.org/wiki/Solanum_vestissimum. Accessed 25 Dec 2017

387. http://tropical.theferns.info/viewtropical.php?id=Solanum+vesti ssimum. Accessed 25 Dec 2017

388. https://en.wikipedia.org/wiki/Solanum_villosum. Accessed 25 Dec 2017

389. http://florida.plantatlas.usf.edu/Plant.aspx?id=56. Accessed 25 Dec 2017

390. http://tropical.theferns.info/viewtropical.php?id=Solanum+viola ceum. Accessed 25 Dec 2017

391. F.R. Chang, C.T. Yen, M. El-Shazly, C.Y. Yu, Bioorg. Med. Chem. Lett. 23, 2738-2742 (2013)

392. C.T. Yen, C.L. Lee, F.R. Chang, T.L. Hwang, H.F. Yen, C.J. Chen, S.L. Chen, J. Nat. Prod. 75, 636-643 (2012)

393. G.S. Raju, M.R. Moghal, S.M.R. Dewan, M.N. Amin, M. Billah, Avic. J. Phytomed. 3, 313-320 (2013)

394. http://www.homeremediess.com/solanum-xanthocarpum-medic inal-use-and-pictures/. Accessed 28 Dec 2017

395. http://www.himalayawellness.com/herbfinder/solanum-xanth ocarpum.htm. Accessed 28 Dec 2017

396. https://herbpathy.com/Uses-and-Benefits-of-Solanum-Xanth ocarpum-Cid1087. Accessed 28 Dec 2017

397. D. Rani, P.K. Dantu, Nat. Acad. Sci. Lett. 38, 275-279 (2015)

398. S. Khanam, R. Sultana, Int. J. Pharm. Sci. Res. 3, 1057-1060 (2012)

399. G.P. Vadnere, R.S. Gaud, A.K. Singhai, Pharmacol. 1, 513-522 (2008)

400. S. Govindan, S. Viswanathan, V. Vijayasekaran, R. Alagappan, J. Ethnopharmacol. 66, 205-210 (1999)

401. O.M. Singh, K. Subharani, N.I. Singh, N.B. Devi, L. Nevidita, Nat. Prod. Res. 21, 585-590 (2007)

402. G. Shiv, S. Gaherwal, C.S. Shrivastava, N. Wast, Eur. J. Exp. Biol. 5, 81-89 (2015)

403. L.N.C.J. Packia, B.M.S. Bharath, M.A. Anita, B.J. Raja, S. Jeeva, Int. J. Inv. Pharm. Sci. 1, 433-437 (2013)

404. K. Abbas, U. Niaz, T. Hussain, M.A. Saeed, Z. Javaid, A. Idrees, S. Rasool, Acta Poloniae Pharm. 71, 415-421 (2014)

405. D.K. Kajaria, M. Gangwar, D. Kumar, S.A. Kumar, R. Tilak, G. Nath, Asian Pac. J Trop. Biomed. 2, 905-909 (2012)

406. Z. Li, X. Cheng, C.J. Wang, G.L. Li, S.Z. Xia, F.H. Wei, Chin. J. Parasitol. Parasit. Diseases 23, 206-208 (2005)

407. W. Li, X. Huang, C. Qi, S. Liu, O. Kodama, K. Utaka, China Pest. 46, 591-593 (2007)

408. T. Changbunjong, W. Wongwit, S. Leemingsawat, Y. Tongtokit, V. Deesin, S. Asian J. Trop. Med. Pubic Health 41, 320-325 (2010)

409. T. Hussain, R.K. Gupta, K. Sweety, M.S. Khan, M.D. Hussain, A.M.D. Sarfaraj, Asian Pac. J Trop. Biomed. 2, 454-460 (2012)

410. G.B. Jalali, H. Ghaffari, H.S. Prakash, K.R. Kini, Pharm. Biol. 52, 1060-1068 (2014)

411. R.K. Gupta, T. Hussain, G. Panigrahi, A. Das, G.N. Singh, K. Sweety, Asian Pac. J Trop. Med. 4, 964-968 (2011)

412. K. Poongothai, P. Ponmurugan, K.S.Z. Ahmed, B.S. Kumar, Asian Pac. J Trop. Med. 4, 778-785 (2011)

413. D.M. Kar, L. Maharana, S. Pattnaik, G.K. Dash, J. Ethnopharmacol. 108, 251-256 (2006)

414. C. Archana, J. Jacob, Asian J. Phytomed Clin. Res. 3, 32-36 (2015)

415. P. Velu, P. Iyappan, A. Vijayalakshmi, D. Indumathi, Biomed. Pharmacother. 84, 430-437 (2016)

416. S. Kumar, A.K. Pandey, BMC Comp. Altern. Med. 14, 112 (2014)

417. S. Kumar, U.K. Sharma, A.K. Sharma, A.K. Pandey, Cell. Mol. Biol. 58, 174-181 (2012)
418. K.K. Bhutani, A.T. Paul, W. Fayad, S. Linder, Phytomedicine 17, 789-793 (2010)

419. M.T. Rahman, M. Ahmed, M. Alimuzzaman, J.A. Shilpi, Fitoterapia 74, 119-121 (2003)

420. T. Hussain, R.K. Gupta, K. Sweety, B. Eswaran, M. Vijayakumar, C.V. Rao, Asian Pac. J. Trop. Med. 5, 686-691 (2012)

421. K.P. Mahesh, K. Murugan, K. Kovendan, J. Subramaniam, D. Amaresan, Parasit. Res. 110, 2541-2550 (2012)

422. K.P. Mahesh, K. Murugan, K. Kovendan, C. Panneerselvam, Parasit. Res. 111, 609-618 (2012)

423. S.K. Bansal, K.V. Singh, S. Kumar, J. Environ. Biol. 30, $221-$ $226(2009)$

424. L. Mohan, P. Sharma, C.N. Srivastava, J. Environ. Biol. 26, 366-401 (2005)

425. K.M. Parmar, P.R. Itankar, A. Joshi, S.K. Prasad, J. Ethnopharmacol. 198, 158-166 (2017)

426. D. Ranka, M. Aswar, U. Aswar, B.S. Bodhankar, Indian J. Exp. Biol. 51, 833-839 (2013)

427. B. Bhatt, J. Chem. Pharm. Res. 3, 176-181 (2011)

428. P.K. Patel, M.A. Patel, B.A. Vyas, D.R. Shah, T.R. Gandhi, J. Ethnopharmacol. 144, 160-170 (2012)

429. O.M. Singh, T.P. Singh, J. Sci. Ind. Res. 69, 732-740 (2010)

430. A.C. Cuervo, B. Gerald, A.V. Patel, Phytochemistry 30, 13391341 (1991)

431. W. Putalun, L.J. Xuan, H. Tanaka, Y. Shoyama, J. Nat. Prod. 62, 181-183 (1999)

432. X.M. Zuo, L.Z. Guo, F. Cheng, Z.Y. Guo, K. Zou, Nat. Prod. Res. Dev. 25, 1205-1208 (2013)

433. Y.Y. Lee, F. Hashimoto, S. Yahara, T. Nohara, N. Yoshida, Chem. Pharm. Bull. 42, 707-709 (1994)

434. A. Mohy-ud-din, Z. Khan, M. Ahmad, M.A. Kashmiri, Asian J. Chem. 22, 2919-2927 (2010)

435. W. Shu, Y. Zhang, W. Ye, G. Zhou, J. Jinnan Univ. Nat. Sci. Med. 32, 493-497 (2011)

436. W. Shu, C. Wu, Y. Zhang, W.C. Ye, G. Zhou, Nat. Prod. Res. 27, 1982-1986 (2013)

437. L.J. Hao, S. Wang, J.J. Zhu, Z.M. Wang, S.H. Wei, China J. Chin. Mat. Med. 39, 2034-2038 (2014)

438. R.H. Al-Sofany, O.A. Rashwan, Bull. Fac. Pharm. 39, 99-101 (2001)

439. K.M. Ahmed, Egyptian J. Pharm. Sci. 37, 37-44 (1996)

440. A.C. Suthar, R.M. Mulani, Pharmacogn. Mag. 4, 112-115 (2008)

441. K.E. Hellenas, A. Nyman, P. Slanina, L. Loof, J. Gabrielsson, J. Chromatogr. 573, 69-78 (1992)

442. R.F. Keeler, D.C. Baker, W. Gaffield, Offic. J. Int. Soc Toxicon. 28, 873-884 (1990)

443. E.A. Mona, H. Miyashita, T. Ikeda, J.H. Lee, H. Yoshimitsu, T. Nohara, K. Murakami, Chem. Pharm. Bull. 57, 747-748 (2009)

444. X. Teng, Y.J. Zhang, C. Yang, Acta Bot. Yunnan. 30, 239-242 (2008)

445. Y. Lu, J. Luo, L. Kong, Mag. Reson. Chem. 47, 808-812 (2009)

446. E.A. Ferro, N.L. Alvarenga, D.A. Ibarrola, I.M.C. Hellion, A.G. Ravelo, Fitoterapia 76, 577-579 (2005)

447. S. Nakamura, M. Hongo, S. Sugimoto, H. Matsuda, M. Yoshikawa, Phytochemistry 69, 1565-1572 (2008)

448. Y.Y. Lu, J.G. Luo, L.Y. Kong, Chin. J. Nat. Med. 9, 30-32 (2011)

449. S. Yahara, T. Yamashita, N. Nozawa, T. Nohara, Phytochemistry 43, 1069-1074 (1996)

450. W. Shu, G. Zhou, W. Ye, Chin. Trad. Herb. Drugs 42, 424-427 (2011)

451. A.P. Colmenares, L.B. Rojas, O.A.C. Mitaine, L. Pouysegu, S. Quideau, T. Miyamoto, C. Tanaka, T. Paululat, Phytochemistry 86, 137-143 (2013)

452. Y. Iida, Y. Yanai, M. Ono, T. Ikeda, T. Nohara, Chem. Pharm. Bull. 53, 1122-1125 (2005) 
453. M. Ohno, K. Murakami, M. El-Aasr, J.R. Zhou, K. Yokomizo, M. Ono, T. Nohara, J. Nat. Med. 66, 658-663 (2012)

454. H. Nawaz, E. Ahmed, A. Sharif, M. Arshad, N. Batool, M.A. Rasool, U.H. Mukhtar, Chem. Nat. Comp. 49, 1091-1094 (2014)

455. H.T. Nguyen, V.T. Nguyen, D.T. Nguyen, V.M. Chau, Tap Chi Duoc Hoc 48, 31-36 (2008)

456. W. Putalun, N. Fukuda, H. Tanaka, Y. Shoyama, J. Liq. Chrom. Rel. Technol. 25, 2387-2398 (2002)

457. A. Schwarz, E. Pinto, M. Haraguchi, C.A. de Oliveira, M.M. Bernardi, S.H. Souza, Phytother. Res. 21, 1025-1028 (2007)

458. J.J. Jared, L.K. Murungi, B. Torto, Pest Manag. Sci. 72, 828-836 (2016)

459. M.T.F. Cornelius, C.C.F. Alves, T.M.S. da Silva, K.Z. Alves, M.G. de Carvalho, Rev. Bras. Farmacia 85, 57-59 (2004)

460. F.J.M. Barbosa, M.F. Agra, R.A. Oliveira, M.Q. Paulo, G. Trolin, Memo. Inst. Oswaldo Cruz 86, 189-191 (1991)

461. J. Manosroi, A. Manosroi, P. Sripalakit, Acta Hort. 679, 105-111 (2005)

462. T. Purwanti, Majalah Farmasi Indon. 8, 115-122 (1997)

463. H. Zhou, F. Wang, Z. Fang, China J. Chin. Mat. Med. 36, 20962098 (2011)

464. J. Bhattacharyya, I.J.L.D. Basilio, L.C.S.L. Morais, M.F. Agra, Biochem. Syst. Ecol. 37, 228-229 (2009)

465. F. Njeh, H. Feki, I. Koubaa, N. Hamed, M. Damak, Pharm. Biol. 54, 726-731 (2016)

466. A. Cristea, M. Tanasescu, P.M. Adelina, Farmacia 40, 25-30 (1992)

467. C. Butnaru, L. Vlase, D. Lazar, L. Agoroaei, Farmacia 59, 172 $178(2011)$

468. H. Wintoch, A. Morales, C. Duque, P. Schreier, Nat. Food Chem. 41, 1311-1314 (1993)

469. A. Sammani, E. Shammaa, F. Chehna, Int. J. Pharm. Sci. Rev. Res. 23, 23-27 (2013)

470. H. Ripperger, Phytochemistry 43, 705-707 (1996)

471. A. Maxwell, M. Seepersaud, R. Pingal, D.R. Mootoo, W.F. Reynolds, J. Nat. Prod. 58, 625-628 (1995)

472. C.A. Coy-Barrera, L.E. Cuca-Suarez, I.O. Clara, Acta Biol. 27, 131-134 (2005)

473. E.C.S. Luis, A.C.B. Carlos, C.I. Orozco, Rev. Colomb. Quimica 33, 7-12 (2004)

474. D. Li, Y.L. Zhao, X.J. Qin, L. Liu, X.W. Yang, Y.Y. Chen, X.D. Luo, Nat. Prod. Bioprospect. 6, 225-231 (2016)

475. J.L. Liu, S.C. Chen, F.L. Lu, D.P. Li, Guangxi Plant Catal. 32, 415-418 (2012)

476. X.L. Zhou, X.J. He, G.X. Zhou, W.C. Ye, X.S. Yao, J. Asian Nat. Prod. Res. 9, 517-523 (2007)

477. M. Xie, Y. Zhou, K. Zou, F. Cheng, R. Liu, J. Chin. Med. Mat. 31, 1332-1334 (2008)

478. J. Luo, B. Zhang, D. Deng, J. Chin. Cereals Oils 30, 125-128 (2015)

479. R. Venkatesh, R. Vidya, K. Kalaivani, Int. J. Pharm. Sci. Res. 5, 5283-5287 (2014)

480. H. Li, S.Y. Peng, D.P. Yang, B. Bai, L.P. Zhu, Z.M. Zhao, Chirality 28, 259-263 (2016)

481. R. Mahadev, H. Ramakrishnaiah, V. Krishna, N.N. Kumar, Don. J. Ess. Oil-Bear. Plants 15, 387-391 (2012)

482. X. Nie, L. Zhang, F. Yao, K. Xiao, S. Dai, China J. Chin. Mat. Med. 40, 1514-1517 (2015)

483. L. Zhang, H. Lei, H.Q. Lin, G.S. Li, X.D. Yue, S.J. Dai, Nat. Prod. Res. 29, 1889-1893 (2015)

484. X.D. Yue, F. Yao, L. Zhang, G.S. Li, S.J. Dai, China J. Chin. Mat. Med. 39, 453-456 (2014)

485. S.J. Dai, L. Shen, Y. Ren, Nat. Prod. Res. 23, 1196-1200 (2009)

486. S.M. Yu, H.J. Kim, E.R. Woo, H. Park, Arch. Pharm. Res. 17, $1-4(1994)$
487. P. Yuan, F. Guo, K. Zheng, K. Chen, Q. Jia, Y. Li, Nat. Prod. Res. 30, 1682-1689 (2016)

488. F. Marx, E.H.A. Andrade, J.G. Maia, Food Res. Technol. 206, 364-366 (1998)

489. M. Suarez, C. Duque, H. Wintoch, P. Schreier, J. Agric. Food Chem. 39, 1643-1645 (1991)

490. M. Suarez, C. Duque, J. Agric. Food Chem. 39, 1498-1500 (1991)

491. M. Suarez, C. Duque, C. Bicchi, H. Wintoch, G. Full, P. Schreier, Flavour Frag. J. 8, 215-220 (1993)

492. C. Duque, H. Wintoch, M. Suarez, P. Schreier, Linalool glycosides as aroma precursors in lulo (Solanum vestissimum D.) fruit peelings (Prog. Flavour Precursor. Stud. Proc. Int. Conf. 1993), pp. 279-281

493. H. Feki, I. Koubaa, M. Damak, Mediter. J. Chem. 2, 639-647 (2014)

494. Y.L. Lin, W.Y. Wang, Y.H. Kuo, C.F. Chen, J. Chin. Chem. Soc. 47, 247-251 (2000)

495. S.A. Nirmal, A.P. Patel, S.B. Bhawar, S.R. Pattan, J. Ethnopharmacol. 142, 91-97 (2012)

496. Y. Zhao, F. Liu, H. Lou, Chin. Trad. Herb. Drugs 33, 555-556 (2010)

497. A. Mohy-Ud-Din, Z. Khan, M. Ahmad, M.A. Kashmiri, S. Yasmin, H. Mazhar, J. Chilean Chem. Soc. 54, 486-490 (2009)

498. W.W. Tang, X.L. Liu, D.Q. Zeng, J. Jiangxi Agric. Univ. 34, 483-486 (2012)

499. Y. Ji, Y. Sun, C. Hui, Lishizen Med. Mat. Med. Res 20, 2431 $2432(2009)$

500. D. Lin, Y. Sun, L. Zhang, X. Zeng, Lishizen Med. Mat. Med. Res 20, C3-C4 (2009)

501. A.C. Ramos, R.O. Rodrigo, Nat. Prod. Res. 31, 2405-2412 (2017)

502. U.W. Hawas, G.M. Soliman, L.T.E. Abou, A.R.H. Farrag, J. Biosci. 68, 19-28 (2013)

503. M.M. Radwan, A. Badawy, R. Zayed, H. Hassanin, M.A. ElSohly, Med. Chem. Res. 24, 1326-1330 (2015)

504. A. Badawy, R. Zayed, S. Ahmed, H. Hassanean, J. Nat. Prod. 6, 156-167 (2013)

505. Y. Wang, Z. Li, J. Yang, J. Yunnan Univ. 20, 396-398 (1998)

506. http://www.motherherbs.com/solanum-indicum.html. Accessed 25 Sept 2017

507. Y. Ren, L. Shen, S. Dai, China J. Chin. Mat. Med. 34, 721-723 (2009)

508. G. Ishii, M. Mori, Y. Umemura, S. Takigawa, S. Tahara, Nippon Shokuhin Kagaku Kogaku Kaishi 43, 887-895 (1996)

509. W. Dan, G.W. Chen, C.R. Han, W.J. Liu, H.Z. Yang, Chin. Trad. Herb. Drugs 43, 1068-1070 (2012)

510. J. Cardona, E.C. Juliana, S. Cuca, E. Luis, G. Barrera, A. Jaime, Rev. Colomb. Quimica 40, 185-200 (2011)

511. M. Ono, Y. Shiono, T. Tanaka, C. Masuoka, S. Yasuda, T. Ikeda, T. Nohara, J. Nat. Med. 64, 500-505 (2010)

512. S. Siqueira, F.S. Vivyanne, M.F. Agra, C. Dariva, S.J.P. Siqueira, J. Sup. Flu. 58, 391-397 (2011)

513. S.T.M.S. Silva, R. Braz-Filho, M.G. Carvalho, M.F. Agra, Biochem. Syst. Ecol. 30, 479-481 (2002)

514. H.L. Yin, J. Li, Q.S. Li, J.X. Dong, Bull. Acad. Milit. Med. Sci. 34, 65-67 (2010)

515. H.J. Huang, H.M. Wang, A.C. Cao, C.X. Zhang, S.H. Wei, T.J. Ling, Nat. Prod. Res. 31, 1831-1835 (2014)

516. Q. Shao, L. Chang, Y. Wei, Z. Wei, J. Chromatogr. Sci. 56, 695-701 (2018)

517. J. He, X.J. Zhang, B.Z. Ma, F. Liu, Chin. Pharm. J. 50, 2035$2038(2015)$

518. J.H. Li, H.L. Yin, J.X. Dong, Acad. Milit. Med. Sci. 37, 130$134(2013)$ 
519. A.K. Chakravarty, S. Mukhopadhyay, S. Saha, S.C. Pakrashi, Phytochemistry 41, 935-939 (1996)

520. L. Wang, N. Wang, X. Yao, Chin. Trad. Herb. Drugs 30, 792794 (2007)

521. A. Simaratanamongkol, K. Umehara, H. Niki, H. Noguchi, P. Panichayupakaranant, J. Funct. Foods 11, 557-562 (2014)

522. X. Zuo, Z. Deng, Z. Guo, K. Zou, F. Cheng, J. Cent, China Norm. Univ. 46, 322-324 (2012)

523. G. Xie, W. Duan, B. Tao, C. Li, Nat. Prod. Res. Dev. 20(627629), 643 (2008)

524. H. Zhou, F. Wang, Y. He, J. Tang, Z. Fang, West China J. Pharm. Sci. 26, 522-524 (2011)

525. E. Rodrigues, L.R.B. Mariutti, A.Z. Mercadante, J. Agric. Food Chem. 61, 3022-3029 (2013)

526. D. Wu, Z. Fang, Guangdong Med. Coll. J. 24, 139-140 (2008)

527. A.K. Sharma, M.C. Sharma, M.P. Dobhal, Pharm. Lett. 5, 355-361 (2013)

528. V. Aeri, Rajkumari, M. Mujeeb, M. Ali, Indian J. Nat. Prod. 21, 40-42 (2005)

529. G.A. Mathis, A. Toggenburger, R. Pokorny, S. Autzen, R. Ibanez, J. Steroid Biochem. Mol. Biol. 144, 40-43 (2014)

530. H. Bachmann, S. Autzen, U. Frey, U. Wehr, Brit. Poultry Sci. 54, 642-652 (2013)

531. D.R. Baek, M.J. Lee, N.I. Baek, K.H. Seo, Y.H. Lee, J. Appl. Biol. Chem. 59, 103-106 (2016)

532. M.G. Morais, A.F.C. Guilherme, A.A. Aleixo, L.A.R.S. Lima, Nat. Prod. Res. 29, 480-483 (2015)

533. P.L. Yuan, X.P. Wang, K.X. Chen, Y.M. Li, Q. Jia, Chin. Patent Med. 38, 104-107 (2016)

534. M.C.D. Ricardo, C.S.L. Enrique, P. Orozco, I. Clara, Actual. Biol. 27, 49-52 (2005)

535. Y.H. Long, J.H. Li, J. Li, B. Li, L. Chen, J.X. Dong, Fitoterapia 84, 360-365 (2013)

536. Y.H. Long, L.J. Hui, B. Li, L. Chen, J.X. Dong, J. Asian Nat. Prod. Res. 16, 153-157 (2014)

537. J.M. Antonio, J.S. Gracioso, W. Toma, L.C. Lopez, F. Oliveira, A.R.M.S. Brito, J. Ethnopharmacol. 95, 83-88 (2004)
538. T. Chen, J. Zhang, X. Sun, X. Xu, S. Zhang, Fine Chem. 26, 47-50 (2009)

539. https://species.wikimedia.org/wiki/Solanum_elaeagnifolium. Accessed 13 Sept 2017

540. http://www.cabi.org/isc/datasheet/50516. Accessed 13 Sept 2017

541. https://garden.org/plants/view/146851/Natri-Solanum-ligustrinu $\mathrm{m} /$. Accessed 28 Sept 2017

542. https://garden.org/plants/photo/357234/. Accessed 28 Sept 2017

543. https://en.wikipedia.org/wiki/Solanum_lycocarpum. Accessed 29 Sept 2017

544. https://en.wikipedia.org/wiki/Solanum_muricatum. Accessed 06 Nov 2017

545. http://www.pfaf.org/user/plant.aspx?latinname=Solanum + muric atum. Accessed 06 Nov 2017

546. https://www.shootgardening.co.uk/plant/solanum-muricatum. Accesed 06 Nov 2017

547. https://www.vanmeuwen.com/p/solanum-muricatum-mini-melon /v17830VM. Accessed 06 Nov 2017

548. P.M. Veira, L.P.M. Marinho, S.C.S. Ferri, C.C. Lee, Anais Acad. Bras. Cienc. 85, 553-560 (2013)

549. http://www.asklepios-seeds.de/gb/solanum-sessiliflorum-seeds .html. Accessed 15 Nov 2017

550. D.R.L.H. Mascato, M. M. Passarinho, D.M.L. Galeno, R. P. Carvalho, J. Nutr. Met. 364185/1-364185/8 (2015)

551. http://tropical.theferns.info/viewtropical.php?id=Solanum + spira le. Accessed 15 Nov 2017

552. http://www.hear.org/pier/species/solanum_torvum.htm. Accessed 6 Dec 2017

553. T.B. Nguelefack, H. Mekhfi, A.B. Dongmo, T. Dimo, P. Watcho, J. Zoheir, J. Ethnopharmacol. 124, 592-599 (2009)

554. G.T. El-Sherbini, R.A. Zayed, E.T. El-Sherbini, J. Parasit. Res. 2009, (2009)

555. N. Chowdhury, A. Ghosh, G. Chandra, B.M.C. Comp, Altern. Med. 8, 10 (2008) 